NIST GCR 15-997

\title{
Measuring the PMI Modeling Capability in CAD Systems: Report 1 - Combined Test Case Verification
}

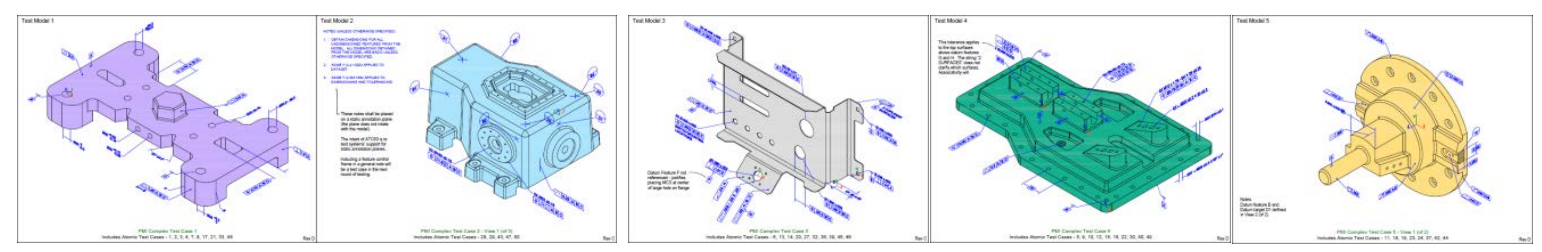

Douglas C. Cheney International TechneGroup Inc.

Bryan R. Fischer Advanced Dimensional Management LLC

This publication is available free of charge from: http://dx.doi.org/10.6028/NIST.GCR.15-997 


\title{
Measuring the PMI Modeling Capability in CAD Systems: Report 1 - Combined Test Case Verification
}

\author{
Prepared for \\ U.S. Department of Commerce \\ Engineering Laboratory \\ National Institute of Standards and Technology \\ Gaithersburg, MD 20899
}

By

Douglas C. Cheney

International TechneGroup Inc.

Bryan R. Fischer

Advanced Dimensional Management LLC

This publication is available free of charge from:

http://dx.doi.org/10.6028/NIST.GCR.15-997

October 2015

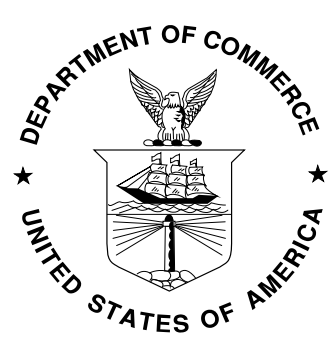

U.S. Department of Commerce

Penny Pritzker, Secretary

National Institute of Standards and Technology

Willie May, Under Secretary of Commerce for Standards and Technology and Director 


\section{Preface}

The National Institute of Standards and Technology (NIST) has created a test system to measure conformance of Computer-Aided Design (CAD) software to American Society of Mechanical Engineers (ASME) standards for product and manufacturing information (PMI), specifically geometric dimensioning and tolerancing (GD\&T) information. The test system has three main components: test cases, test CAD models, and verification and validation test results. The verification and validation results measure PMI implementation capabilities in CAD software and derivative STEP, JT, and 3D PDF files.

All of the test cases, test models, test results, and other presentations are available from the project website: http://www.nist.gov/el/msid/infotest/mbe-pmi-validation.cfm

This report is the first of three reports about the test system. The reports can be read independently of each other.

- Measuring the PMI Modeling Capability in CAD Systems: Report 1 - Combined Test Case Verification

- Measuring the PMI Modeling Capability in CAD Systems: Report 2 - Test Case Validation

- Measuring the PMI Modeling Capability in CAD Systems: Report 3 - Fully-Toleranced Test Case Verification

\section{Disclaimers}

The reports were prepared for the Engineering Laboratory of the National Institute of Standards and Technology under the following contracts:

- SB1341-12-SE-0860, RECON Services Inc., "PMI Conformance Testing Models"

- SB1341-12-SE-0853, International TechneGroup Inc., "PMI and Composite Information Validation and Conformance Testing”

- SB1341-14-SE-0061, International TechneGroup Inc., "PMI Test Cases and Models, Validation and Conformance Testing”

The contents of the reports do not necessarily reflect the views of NIST. NIST and the authors do not make any warranty, expressed or implied, nor assume any legal liability or responsibility for the accuracy, completeness, or usefulness of any information, product, or process included in the reports.

Any mention of commercial products is for information purposes only; it does not imply recommendation or endorsement by NIST. The test system can be used without any restrictions. Its use in other software or hardware products does not imply a recommendation or endorsement by NIST of those products.

\section{Project Participants}

- International TechneGroup Inc. (ITI) - test model creation, expert review, verification, validation, and documentation

- Advanced Dimensional Management LLC - test case definition and expert review

- $\quad$ RECON Services Inc., Neilsoft Ltd. - test model creation and expert review

- Department of Energy Kansas City Plant (operated by Honeywell FM\&T), RECON Services Inc., Sigmetrix - expert review

Cover image: Combined test cases 


\section{Table of Contents}

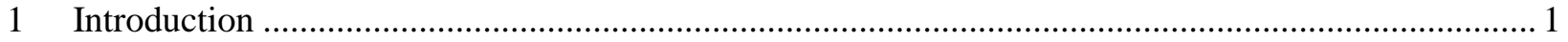

$1.1 \quad$ Model-Based Enterprise and Model-Based Definition ..............................................................................

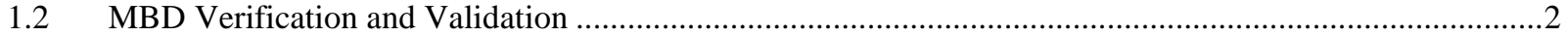

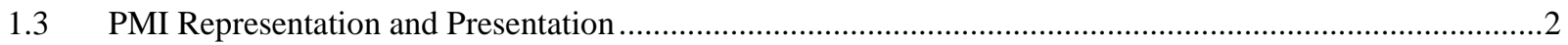

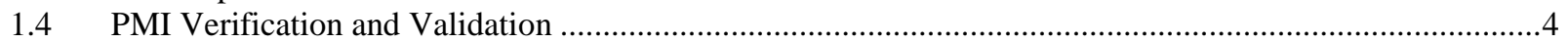

2 Methodology for PMI Modeling Capability Assessment ................................................................ 5

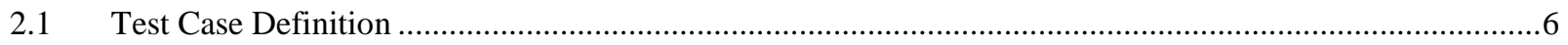

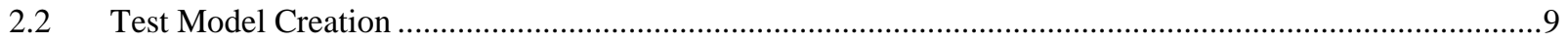

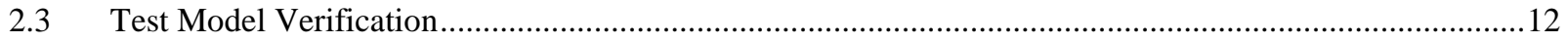

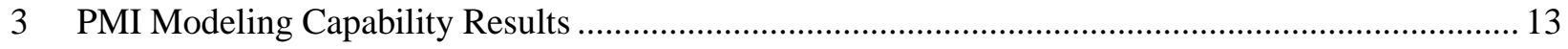

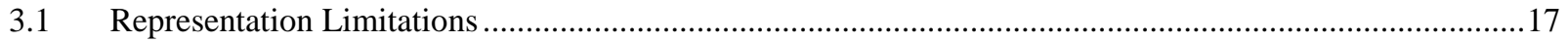

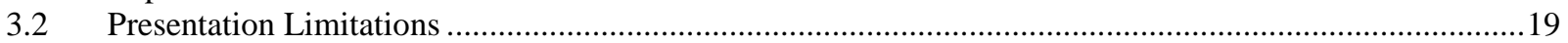

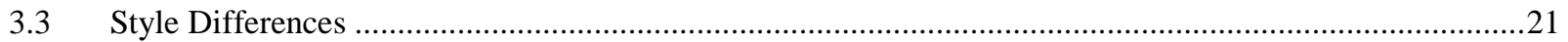

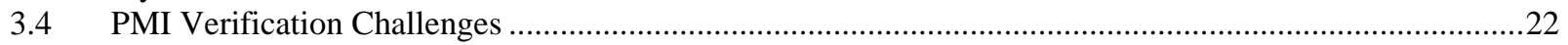

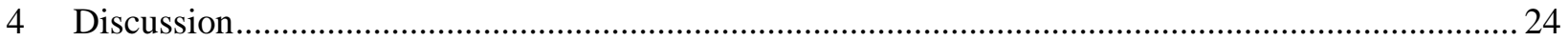

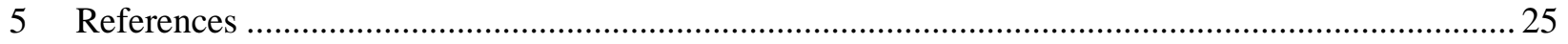

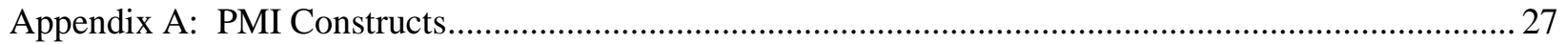

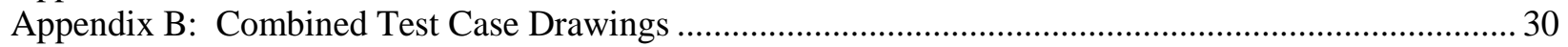

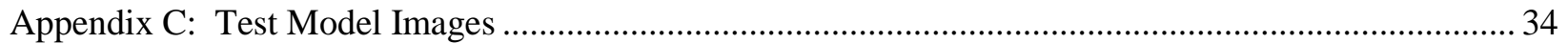

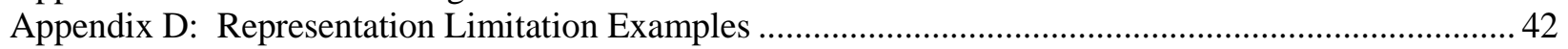

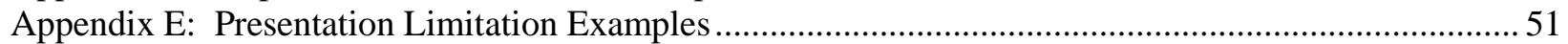

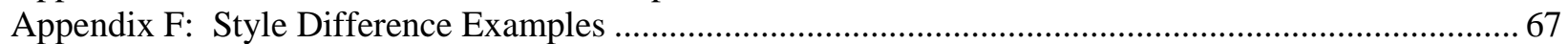




\section{List of Figures}

Figure 1: Methodology for PMI modeling capability assessment ......................................................... 5

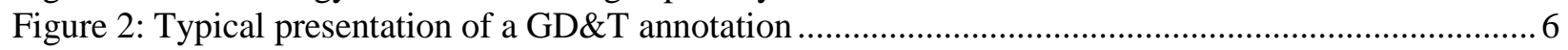

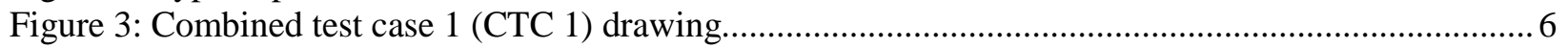

Figure 4: Combined test case 2 (CTC 2) drawing....................................................................... 7

Figure 5: Combined test case 3 (CTC 3) drawing........................................................................ 7

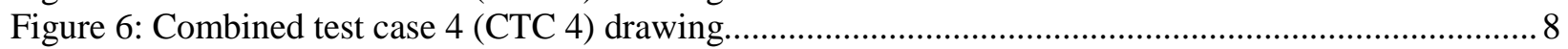

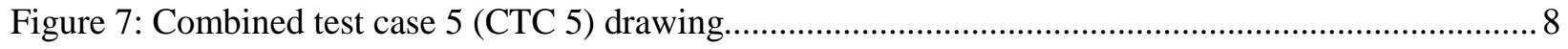

Figure 8: Combined test case 1 (CTC 1) modeled in CATIA V5 R21 ..................................................... 10

Figure 9: Combined test case 1 (CTC 1) modeled in Creo 2.0 ............................................................. 10

Figure 10: Combined test case 1 (CTC 1) modeled in NX 8.0 .............................................................. 11

Figure 11: Combined test case 1 (CTC 1) modeled in SOLIDWORKS 2012 ........................................... 11

Figure 12: PMI modeling capability results by CAD system ................................................................. 14

Figure 13: PMI modeling capability results by CAD system, excluding coordinate system structure

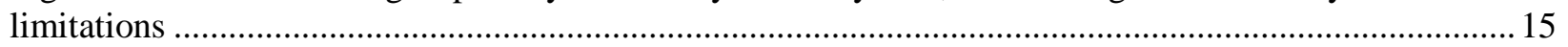

Figure 14: Example of a representation limitation.......................................................................... 17

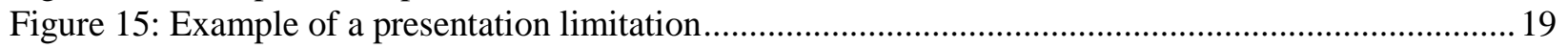

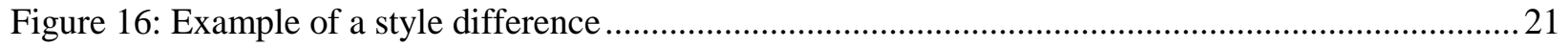

Figure 17: Extension lines represented as separate dimension annotations ...............................................22

Figure 18: Different threaded hole supplemental geometry representations ............................................23

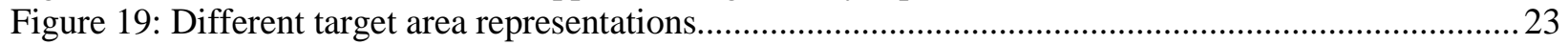

\section{List of Tables}

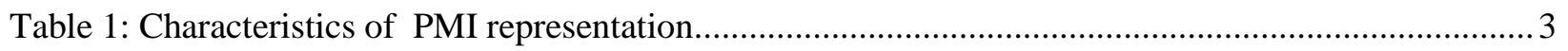

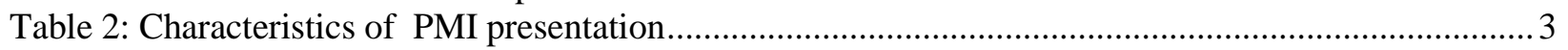

Table 3: PMI element counts by type and test case ............................................................................. 13

Table 4: PMI representation limitations by characteristic and CAD system .......................................... 15

Table 5: PMI presentation limitations by characteristic and CAD system ................................................ 16

Table 6: PMI entity abbreviations..................................................................................................... 17

Table 7: Representation limitation counts by characteristic and type.................................................... 18

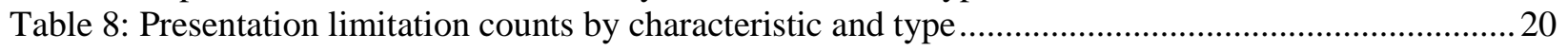

Table 9: Style difference counts by characteristic and type................................................................... 22 


\section{Introduction}

A methodology for measuring the product and manufacturing information (PMI) modeling capability of computer-aided design (CAD) systems has been developed to measure technology readiness and to track progress as functionality gaps are closed. A measurement methodology will enhance the ability of discrete-part manufacturing companies to implement a model-based enterprise (MBE) [1-5]. The use of a clear capability assessment will accelerate MBE technology development by CAD software vendors. This can increase the business opportunities for both manufacturing companies and technology providers.

Common practice in discrete-part manufacturing companies is to use CAD systems to create threedimensional (3D) models that precisely define the shape of their products. The companies derive twodimensional (2D) drawings from the 3D model that detail the product's dimensions, tolerances, and other manufacturing information. Manufacturing organizations have typically considered the drawings to be the master product definition for all downstream processes such as simulation, manufacturing, and inspection. Often a 3D model is recreated from the drawing in one or more downstream processes, especially when performed by external suppliers. In some cases, the original 3D model is released with the drawing as a reference document [6].

As the drawing goes through several engineering changes, the 3D model may become outdated because it is not the master design document. Therefore, model recreation from the drawing tends to increase as a product matures. Downstream consumers of the drawing visually interpret the dimensions, tolerances, and other manufacturing information and manually reenter this information into downstream systems. Manually reentering information is a potentially error-prone process. This human interpretation is repeated for each engineering change.

Global business requirements are driving companies to produce better and cheaper products in less time to market. Management initiatives target the reduction of risk due to variation and the elimination of all non-value-added tasks throughout the engineering, manufacturing, and sustainment phases of a product's lifecycle. A leading process improvement initiative today is the concept of MBE [7].

\subsection{Model-Based Enterprise and Model-Based Definition}

A model-based enterprise (MBE) builds on the foundation that all product data may be integrated into a single model-based definition (MBD). This eliminates the need for 2D drawing generation, the recreation of 3D models, and/or the visual interpretation of drawing data in downstream processes. It improves product quality by eliminating drawing-to-model inconsistencies, unintentional model changes during recreation, and drawing interpretation errors. It decreases overall time to market by enabling direct reuse of the digital product model in downstream software systems.

A key component of an MBD is the integration of all the product and manufacturing information (PMI) into the 3D model. Dimensions, tolerances, notes, and other data previously found on a drawing are displayed in the model with direct links to the affected portion of the model's shape definition or 3D geometry. The data is grouped into multiple saved views to aid visual consumption. More importantly, the visual data is linked to an internal representation that is well defined and structured for automated consumption in downstream software systems. Derivative models, such as STEP (ISO 10303 -known informally as the STandard for Exchange of Product model data) [8-10], JT [11-13] and 3D PDF [14-16] files, are created as needed for downstream consumers who do not have direct access to the CAD system in which the native MBD model is defined. 


\subsection{MBD Verification and Validation}

In a drawing-based product lifecycle, the drawing is manually checked by a person before release and then visually interpreted by a person during downstream reuse. This results in processes that tolerate lowlevel variation in the digital data while being fairly controlled. In a model-based process, the checking task is often eliminated on the assumption that a precise native model should be directly reusable in downstream systems without error. This results in processes that are less tolerant of digital data variation while being less controlled. However, if a company is going to rely on an MBD model throughout its product's lifecycle, the model must be reliable. Therefore, quality checking of the geometry and PMI in the master model, and their equivalent entities in all derivatives, is critical before release to downstream processes.

Various automotive, aerospace, and defense industry groups have identified precise geometry and PMI quality criteria for native MBD models and their derivatives. These include:

- Strategic Automotive Special Interest Group (SASIG) Product Data Quality (PDQ) team [17]

- PDES, Inc. [18] and ProSTEP iViP [19] collaboration for Long-Term Archival (LOTAR) [20]

- Department of Defense's MBE team [1]

Each group has recently documented these requirements in international, regional, and domestic standards such as:

- $\quad$ Managed Model-based 3D Engineering - STEP ISO 10303-242 [21, 22]

- CAD mechanical 3D Explicit geometry information - EN9300-110 [23]

- DoD Standard Practice: Technical Data Packages - MIL-STD-31000A [24]

These groups generally agree that the process of quality checking a native CAD model should be called verification. This process verifies that the product definition data is complete, consistent, and conformant to relevant standards. They recommend that the process of determining whether the data in a derivative model is equivalent to the native model should be called validation. This process validates that all data has been translated with any digital variation within acceptable limits specified by the anticipated downstream processes.

Due to the complexity of MBD data, it is unrealistic to implement verification or validation using an interactive, manual process. Several CAD applications have been developed to automate verification and validation using the criteria referenced above. While these applications make MBD quality control feasible, they impose an important requirement on the CAD modeling systems: that all MBD data, including 3D geometry and PMI, must be accessible through an application programming interface (API) to third-party developers.

\subsection{PMI Representation and Presentation}

An MBD must contain sufficient PMI representation so that automated systems, such as machining and inspection, can reuse the information efficiently and correctly in all downstream processes. PMI representation (also known as semantic PMI) includes all information necessary to represent GD\&T without any graphical presentation elements. The PMI presentation should also be clearly presented for visual (human) consumers so that they understand and trust the model-based definition. PMI presentation (also known as graphical PMI) consists of geometric elements such as lines and arcs preserving the exact appearance (color, shape, positioning) of the GD\&T annotations. The internal PMI representation should be structured and defined so each element is clear, complete, and consistent. The PMI presentation 
should be organized into saved views with annotations that support cross-highlighting of affected geometry.

These two aspects of PMI, representation and presentation, are best understood by considering how their key characteristics are applied to the various components of an MBD. Table 1 and Table 2 list the characteristics of PMI representation and presentation, respectively. The following is an explanation how they apply to the product geometry, coordinate systems, supplemental geometry, annotations, and saved views in an MBD.

\section{Table 1: Characteristics of PMI representation}

\begin{tabular}{|l|}
\hline Annotation structure \\
Annotation parameters \\
Annotation geometry \\
Coordinate system structure \\
Coordinate system parameters \\
Supplemental geometry structure \\
Supplemental geometry parameters \\
\hline
\end{tabular}

\section{Table 2: Characteristics of PMI presentation}

\begin{tabular}{|l|}
\hline Annotation visibility \\
Annotation color \\
Annotation name \\
Annotation layout \\
Annotation location \\
Annotation orientation \\
Annotation lines \\
Annotation text \\
Coordinate system visibility \\
Coordinate system color \\
Coordinate system name \\
Coordinate system text \\
Supplemental geometry visibility \\
Supplemental geometry color \\
Saved view structure \\
Saved view name \\
Saved view frustum \\
\hline
\end{tabular}

MBD product geometry is structured to differentiate the geometric entities that define the 3D shape of the product from other entities used as reference, context, or supplemental geometry for annotations. For most discrete-part product models, a solid (closed volume) or shell (open surface) definition provides the highest level of definition for downstream processes. The parametric definition of the model is complete, correct, and useful for revisioning. The explicit definition of topology and geometry is free of defects that impede downstream reuse. The meta-data properties associated with the product model capture basic product management data, such as ownership and lifecycle state. The visibility status and display color of the product geometry are appropriate for visual interpretation by downstream users.

MBD annotations have a specified type (dimension, feature control frame, note, etc.) and named parameters (nominal value, tolerance, material modifier, etc.) that facilitate automated interpretation downstream. An annotation's associated geometry includes all affected surfaces in the product geometry and any supplemental geometry. It does not include any extraneous geometry. This facilitates both automated consumption and visual interpretation, also known as cross-highlighting. The visibility, layout, location, and orientation of the annotation in saved views, along with its color, display name, lines, and text, are appropriate for visual interpretation by downstream users.

MBD coordinate systems have explicit named associations with the feature control frames that rely on the datum reference frames they represent. Each coordinate system's location and orientation accurately represent the datum reference frame. The coordinate system's visibility in each saved view corresponds 
to the visibility of its associated annotations. Its color, name, and display text are appropriate for visual interpretation by downstream users.

Supplemental geometry is geometric elements that do not belong to the shape of a part. The geometric elements are used to create other shapes or contain information about part features such as hole centerlines. MBD supplemental geometry entities have the correct form or structure for the annotations that references them. For example, the limited area for a datum target defines the portion of the underlying solid face or surface that is inside versus outside. The location, orientation, and size of each supplemental geometry entity complete the conceptual definition of its associated annotations. Its visibility in saved views corresponds to the visibility of its associated annotations. Supplemental geometry color is appropriate for visual interpretation by downstream users.

A saved view facilitates the presentation of the model and associated PMI by defining a subset of the PMI and an orientation from which it is viewed. MBD saved views are structured to contain a related set of annotations, with their associated supplemental geometry and coordinate systems, along with the appropriate product geometry. Each saved view may contain the complete geometric definition of the product or a portion defined by a cross section. The contents of a saved view are displayed within a frustum, or pyramid of vision, that is intuitive for visual interpretation by downstream users.

\subsection{PMI Verification and Validation}

The process of querying PMI data in an MBD model for verification is straightforward as long as the CAD API provides sufficient access to the data. First, the type and properties of each annotation entity are retrieved and compared with those specified in the test case documentation. Second, any relationships between the annotation and other annotations or geometry entities are queried and compared with the specification. Since an MBD model may contain multiple annotations with similar types and properties, it may be necessary also to query the graphic presentation data in order to match reliably each annotation with its specification and to confirm its relationships are correct.

The process of comparing PMI constructs between MBD models in dissimilar CAD systems for equivalence validation is more complex. The primary challenge is to correctly match corresponding annotation entities before comparing their characteristics. Because all of the presentation characteristics can vary significantly without changing the meaning (representation), these cannot be reliably used for matching purposes. The test model images in Figures 8-11 illustrate the typical variation between the CAD systems used for this assessment. Reliable annotation matching requires that all product and supplemental geometry entities be matched. Then, the subset of annotations entities associated with each set of matching geometry entities are matched and compared. Annotations that have been added, removed, or had their geometry associations changed will remain unmatched.

Some PMI constructs make automation of the above verification and validation processes difficult (see section 3.4). The various CAD systems use different modeling methodologies for these constructs that are each considered valid within the ASME standards. Until the CAD systems converge toward common methodologies, or the standards are modified to require this, the MBD verification and validation technologies must implement advanced reasoning and exception handling to accommodate this allowable variation in PMI definition. 


\section{Methodology for PMI Modeling Capability Assessment}

The PMI modeling capability of the CAD systems commonly used by discrete-part manufacturing companies to support MBE was assessed using a formal methodology [25], shown in Figure 1, involving:

1. Test case definition and expert review

2. Test CAD model creation based on the test case definitions

3. Verification of the CAD models against the test case definitions

4. Generation of derivative STEP, JT, and 3D PDF files by the Implementor Forums [12, 14, 26]

5. Validation of the derivative files against the CAD models and test case definitions

This report is concerned with steps 1-3 of the PMI modeling capability assessment. The validation of derivative files for one of the test cases is documented in second report of this series [27]. The verification of other test cases is documented in the third report of this series [28].

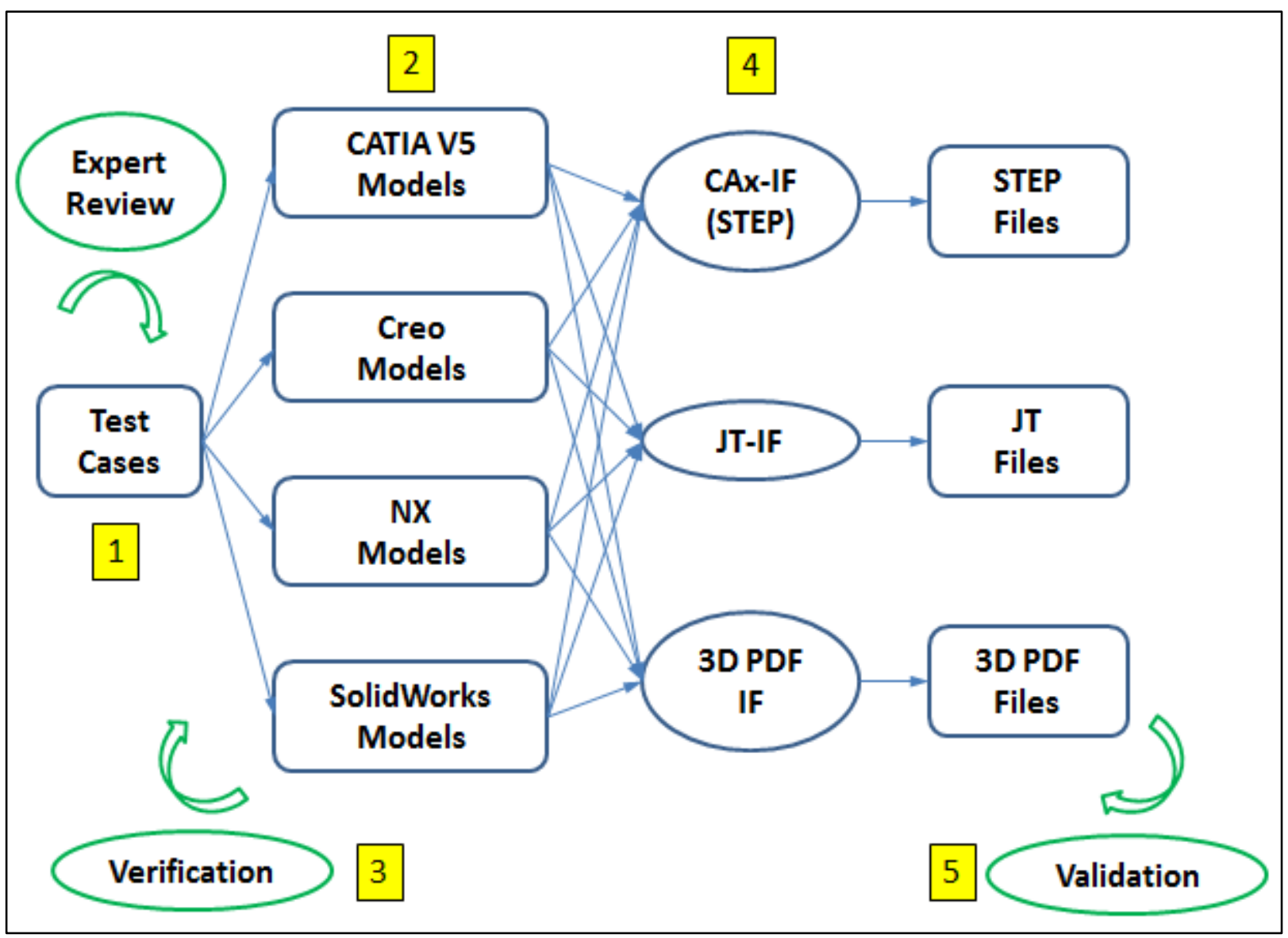

Figure 1: Methodology for PMI modeling capability assessment 


\subsection{Test Case Definition}

For test case generation, an industry expert in geometric dimensioning and tolerancing (GD\&T) defined representative PMI constructs allowed by the American Society of Mechanical Engineers (ASME) standards for 2D drawings Y14.5-1994 [29] and 3D models Y14.41-2003 [30]. (Newer versions of both standards are available.) A PMI construct is a group of annotation entities which define an elemental concept, for example: defining a datum feature with a datum feature symbol (one annotation) or controlling the variation of a hole with a size dimension, a feature control frame, and its associated datum features (3 to 5 annotations). Figure 2 shows the presentation of a typical GD\&T annotation [31].

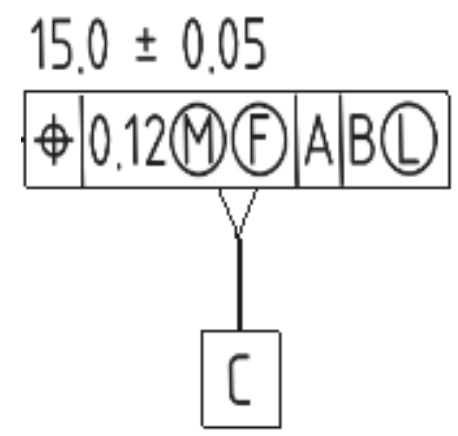

Figure 2: Typical presentation of a GD\&T annotation

The constructs defined for this assessment are listed in Appendix A. The constructs were applied to five discrete-part geometry models, with approximately ten PMI constructs in each model.

Each combined test case (CTC) is documented with a set of drawings and explanatory text, as shown in Figures 3-7. Drawings of other views of each test case are in Appendix B.

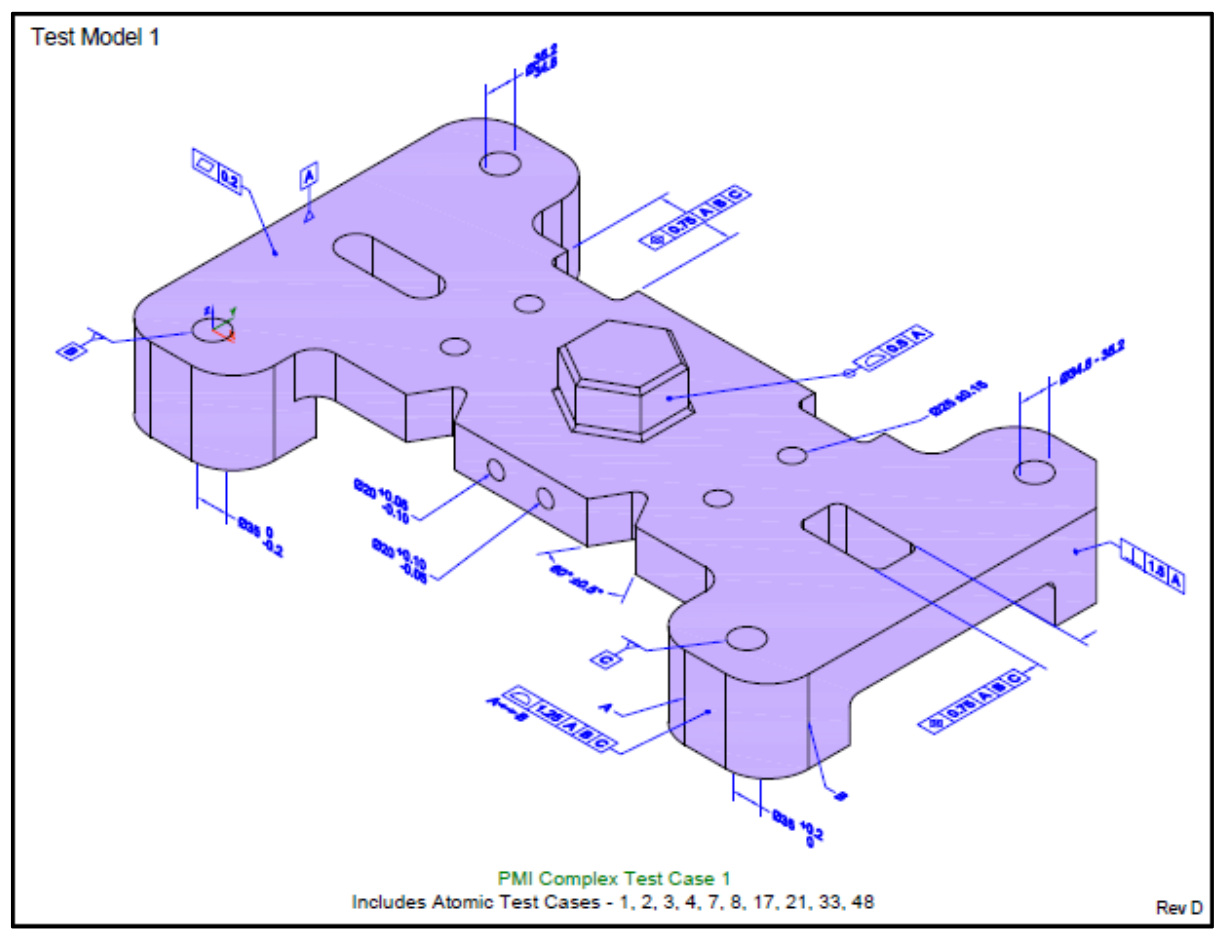

Figure 3: Combined test case 1 (CTC 1) drawing 


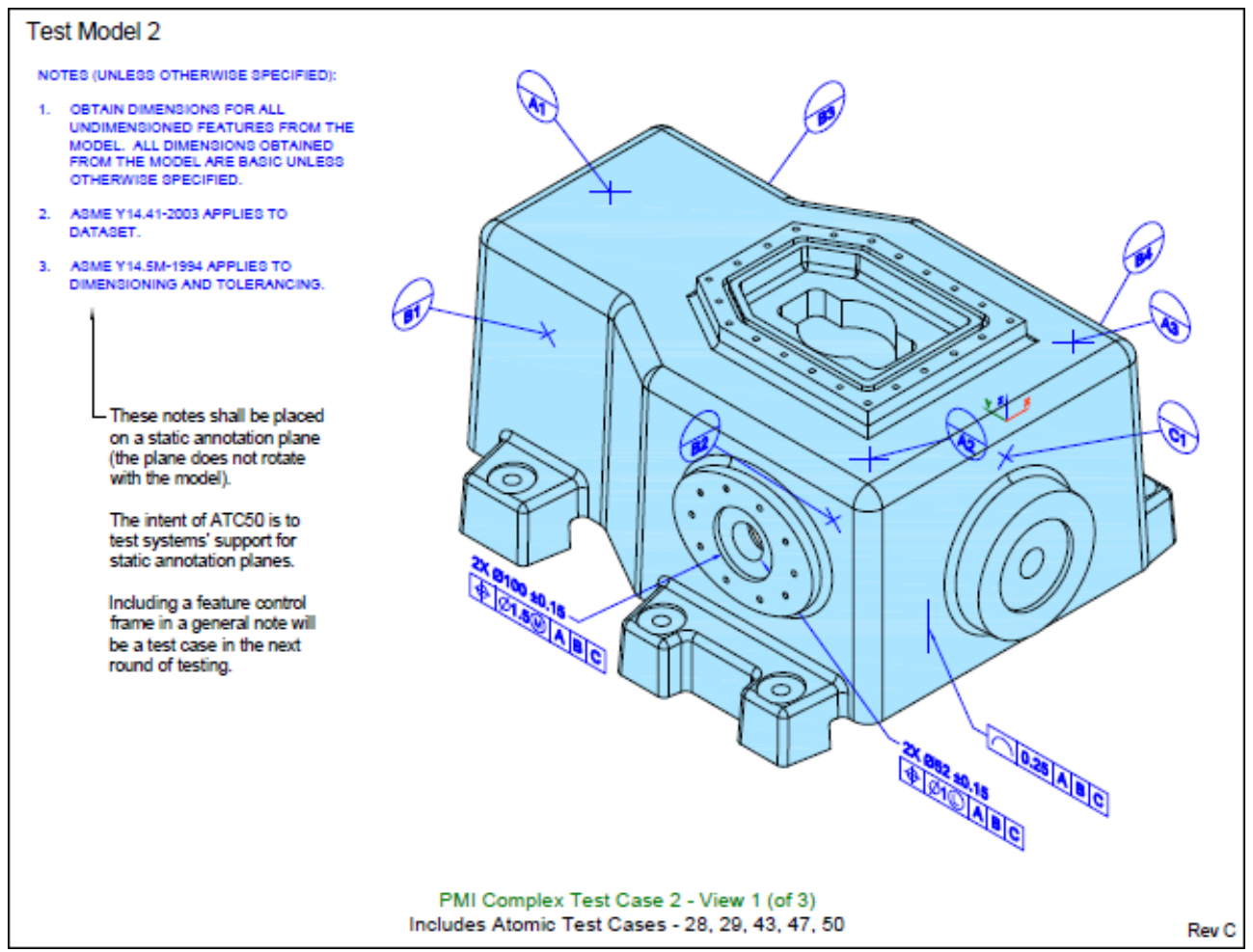

Figure 4: Combined test case 2 (CTC 2) drawing

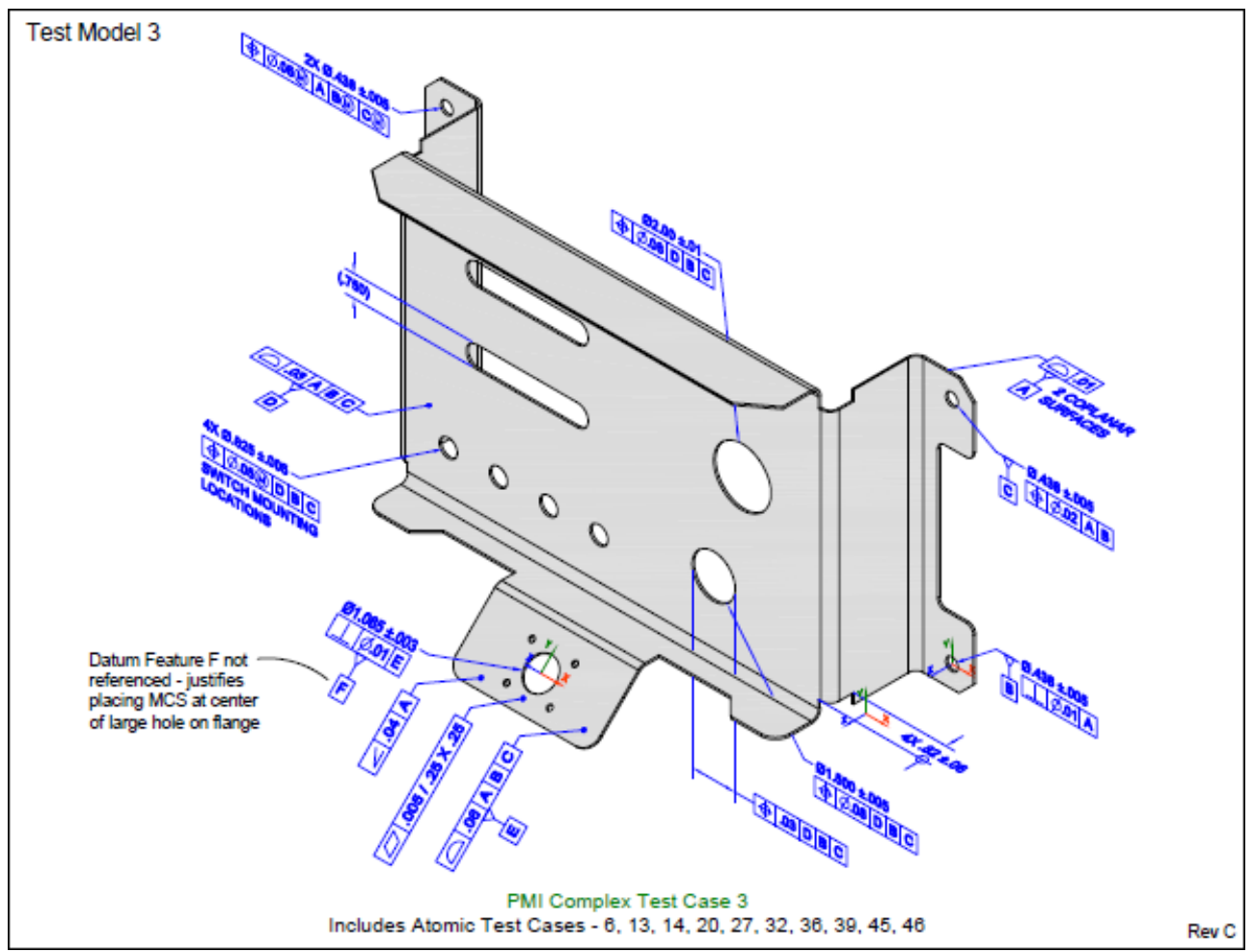

Figure 5: Combined test case 3 (CTC 3) drawing 


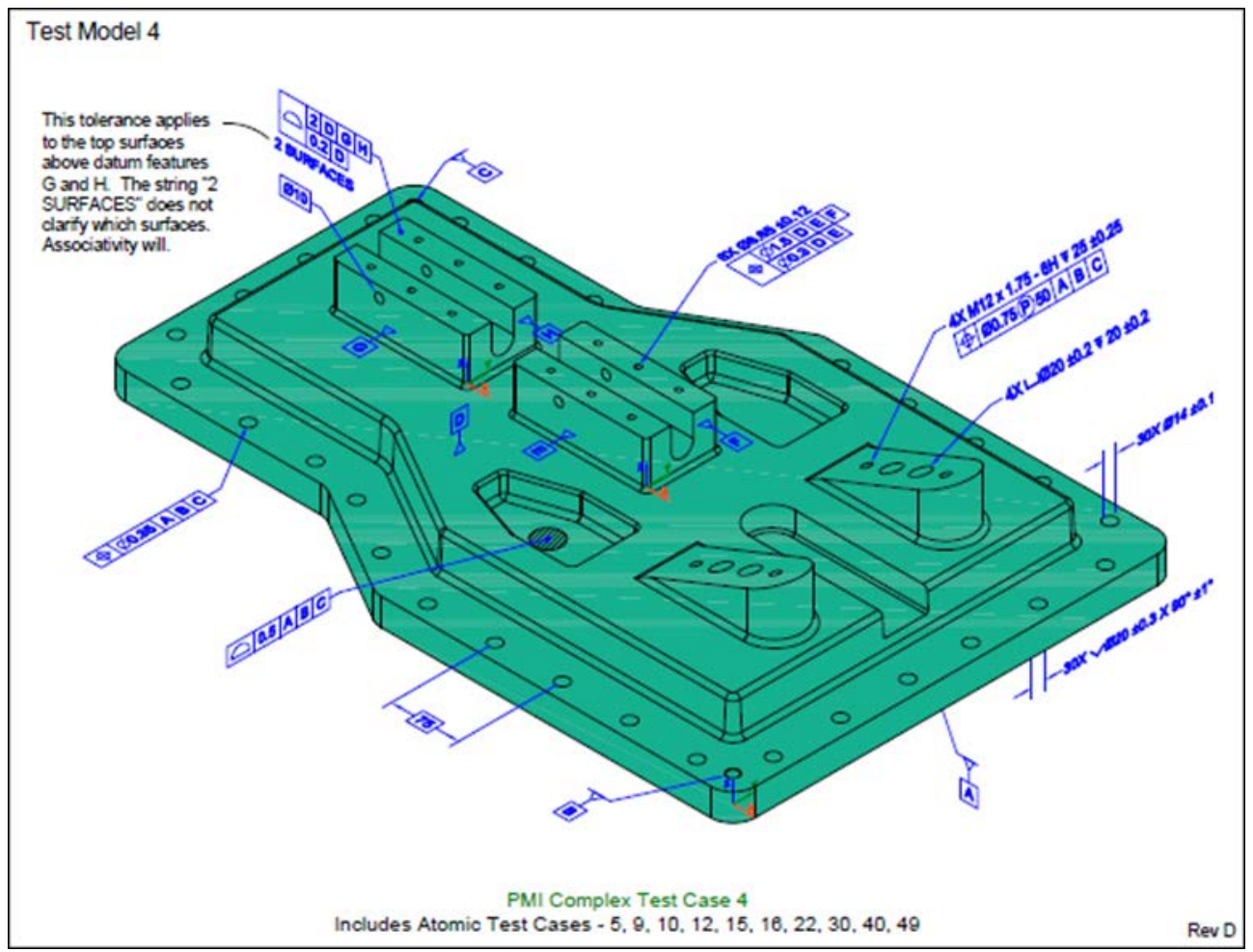

Figure 6: Combined test case 4 (CTC 4) drawing

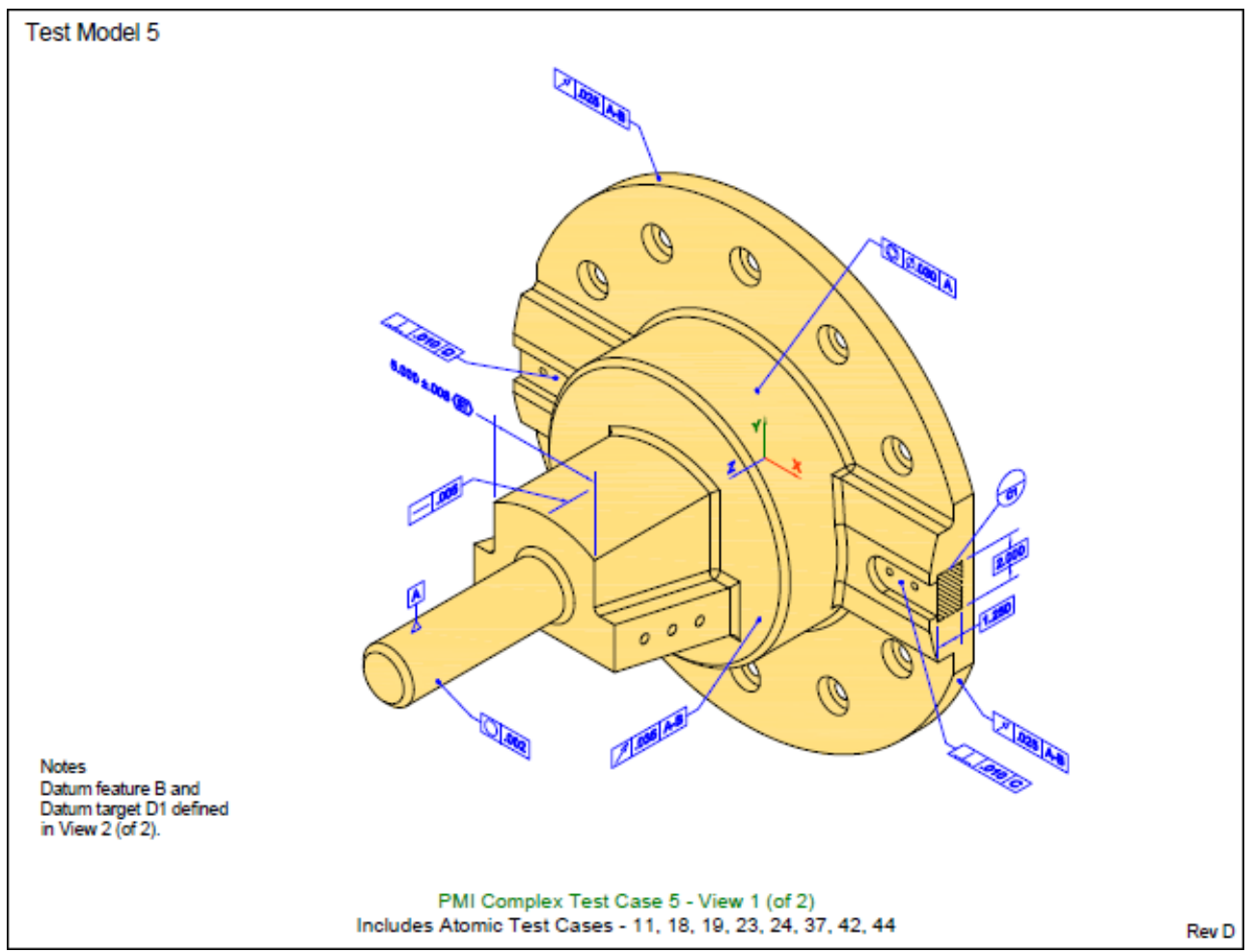

Figure 7: Combined test case 5 (CTC 5) drawing 
Other industry GD\&T experts reviewed the five CTCs for clarity and correctness. The CTCs were refined based on the expert feedback. All experts agreed that the CTCs are intended to simply combine representative constructs and do not define products that are fully-toleranced and/or functional for tolerance purposes. The test cases are also not intended to represent best practice in how to apply GD\&T to a part. Simpler GD\&T strategies could have been used. The test cases are intended to exercise valid presentations of GD\&T defined in the ASME Y14 standards.

\subsection{Test Model Creation}

A team of CAD experts created CAD models for each CTC in four CAD systems that were available in early 2013:

- CATIA V5 R21 from Dassault Systemes [32]

- Creo 2.0 from PTC [33]

- $\quad$ NX 8.0 from Siemens PLM [34]

- SOLIDWORKS 2012 from Dassault Systemes [35]

The CAD experts used the above PMI representation and presentation criteria to create models with equivalent meaning, and negligible graphical variation. When it was not possible to satisfy both sets of criteria, the representation was given precedence over the presentation. Figures 8-11 show combined test case 1 (CTC 1) modeled in each of the four CAD systems. Images of each test model, each with multiple saved views, are shown in Appendix C. 


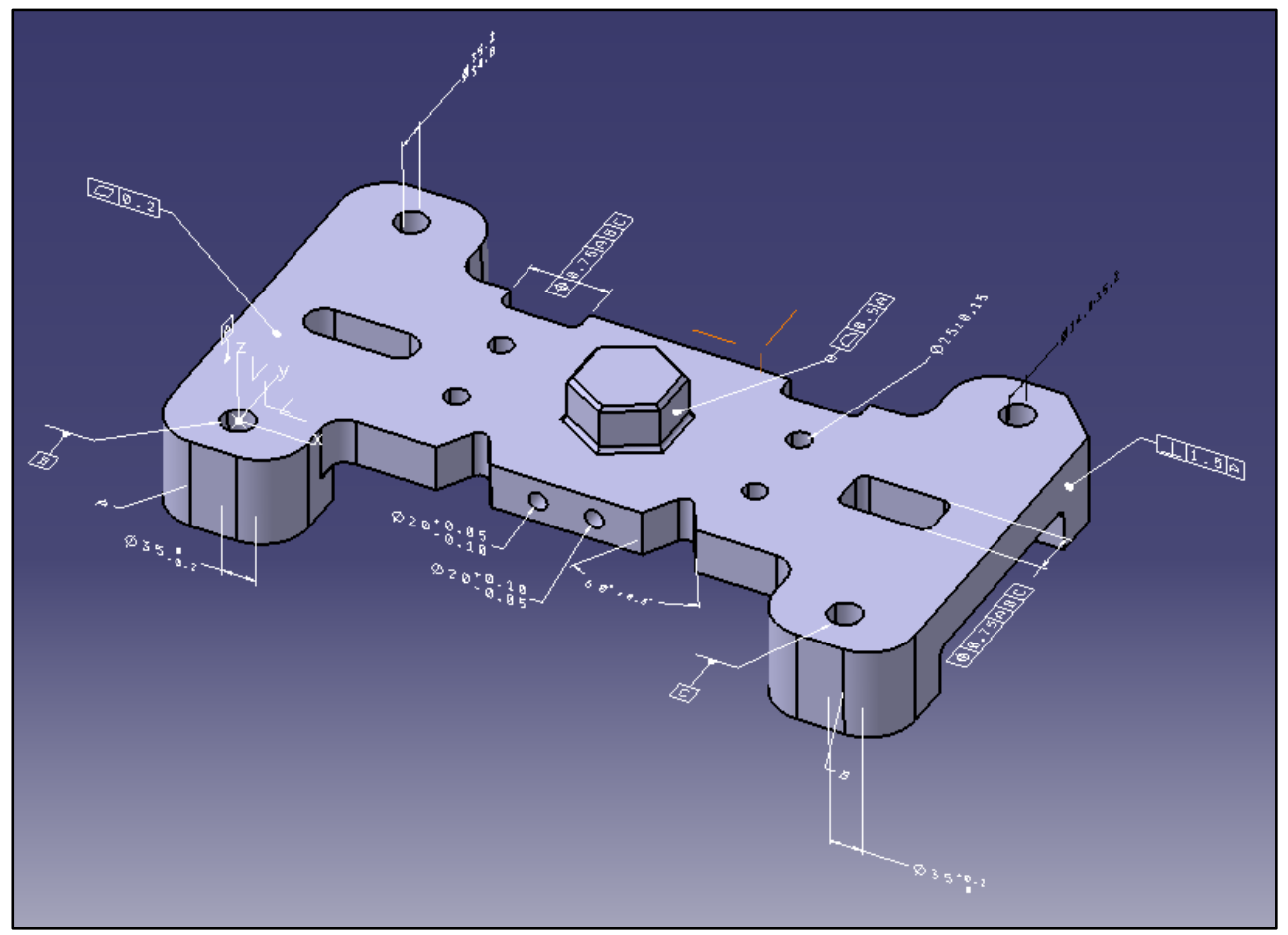

Figure 8: Combined test case 1 (CTC 1) modeled in CATIA V5 R21

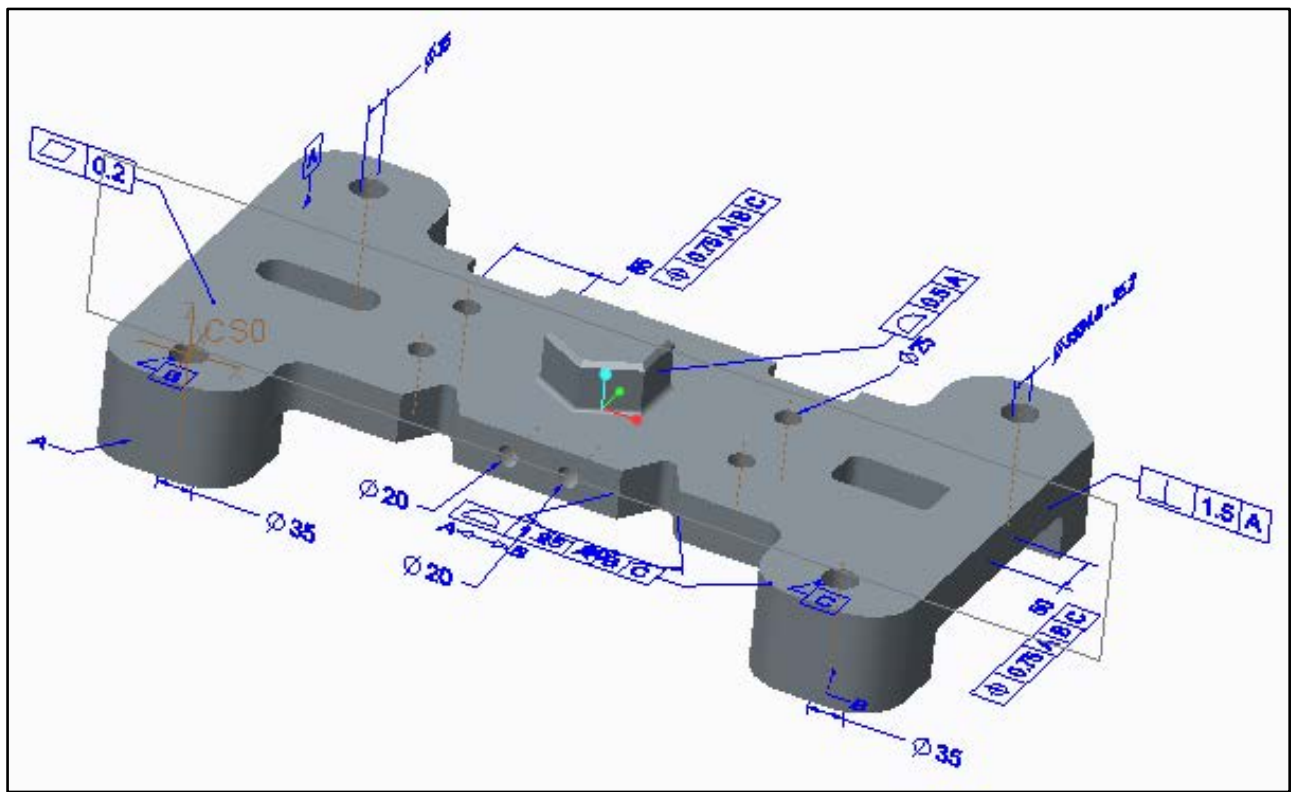

Figure 9: Combined test case 1 (CTC 1) modeled in Creo 2.0 


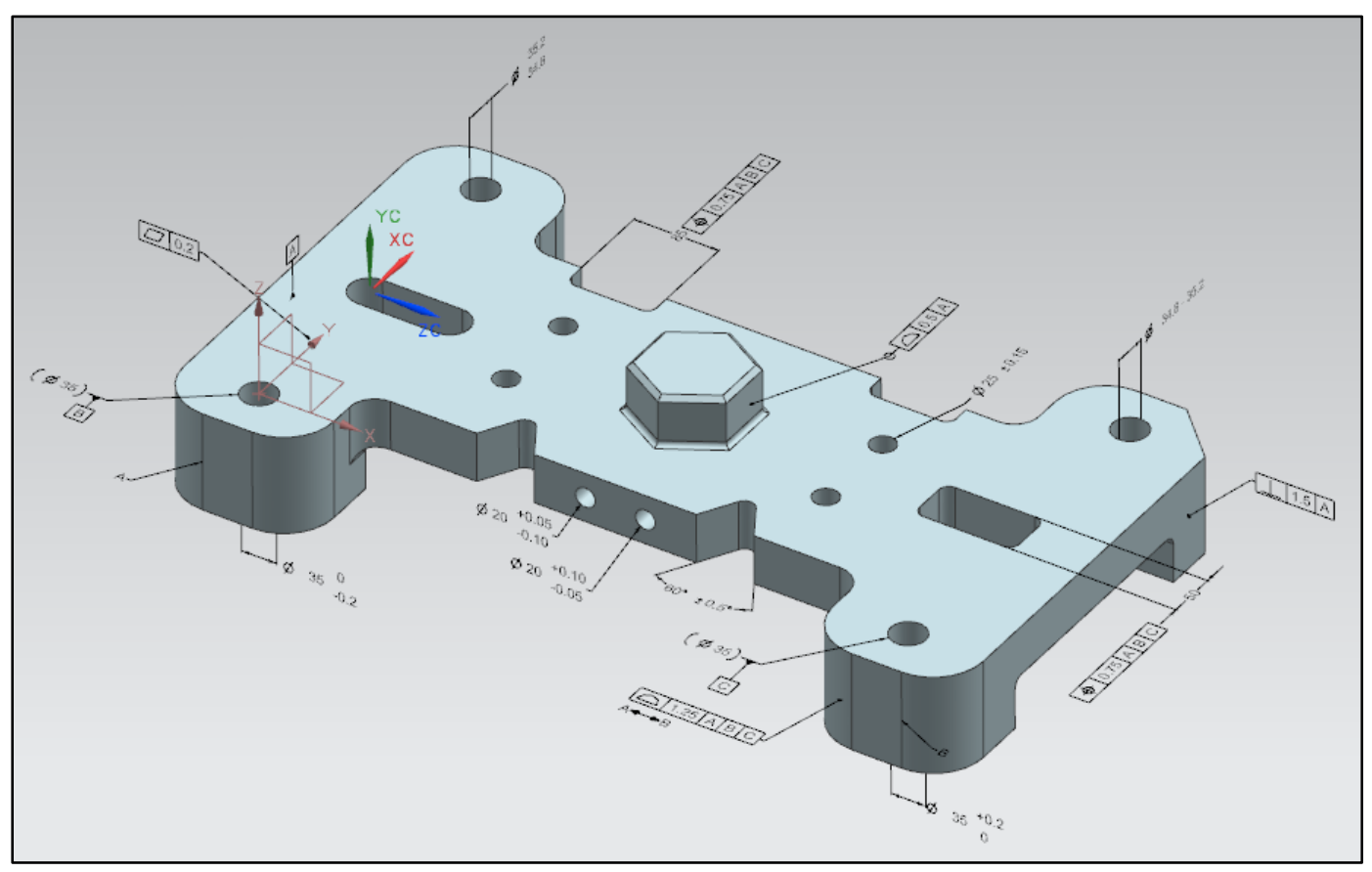

Figure 10: Combined test case 1 (CTC 1) modeled in NX 8.0

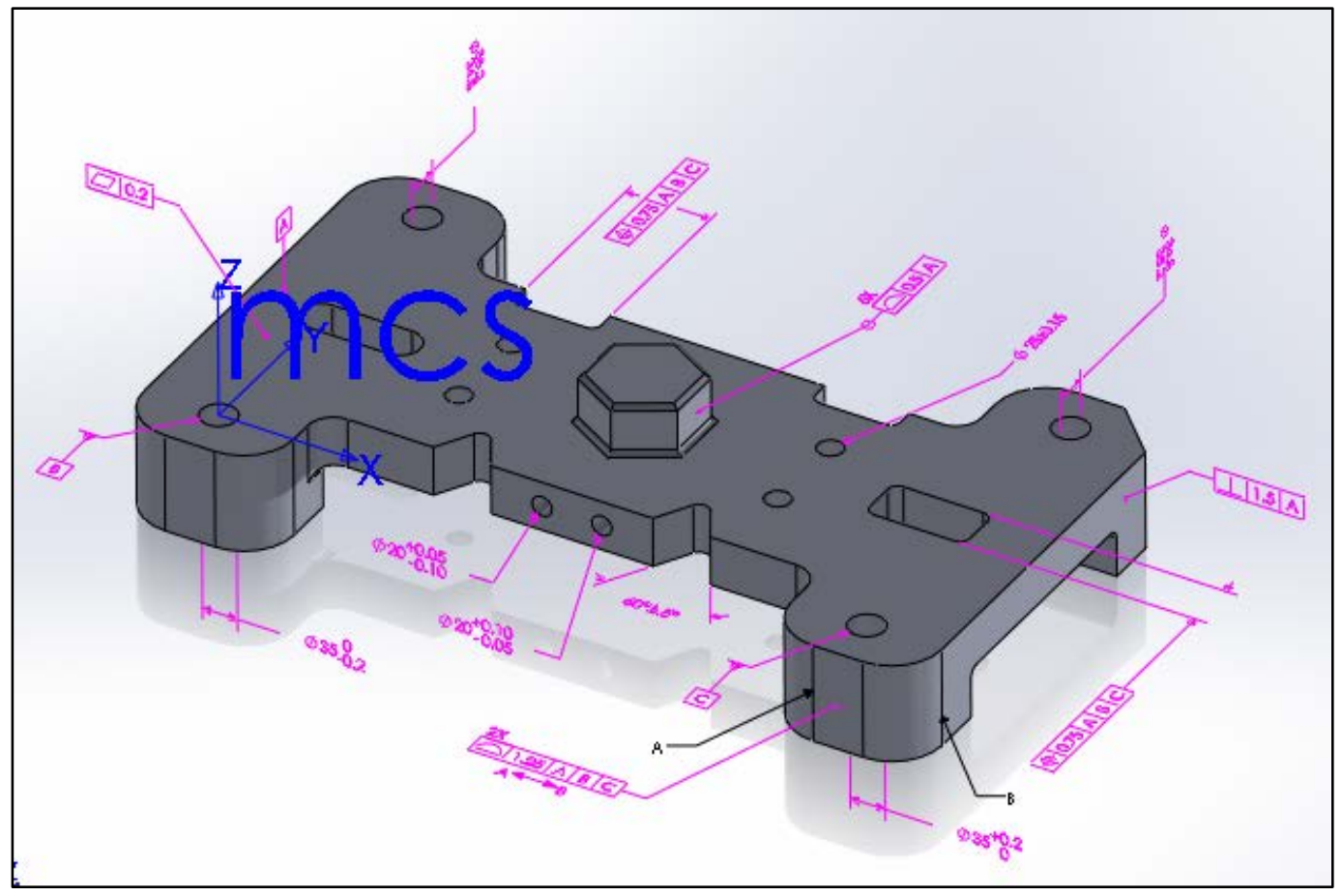

Figure 11: Combined test case 1 (CTC 1) modeled in SOLIDWORKS 2012 


\subsection{Test Model Verification}

The CAD validation software CADIQ 8.0 [36] was used to query the PMI representation and presentation data in a 3D model using the API of each CAD system. The software vendor for CADIQ developed and refined algorithms for matching and comparing each data element between models in different CAD systems that were based on the same test case definition.

After the models were complete, a CAD validation specialist manually compared the data queried for each PMI element in the five models for one CAD system to the five test case definitions. Significant discrepancies or deficiencies were documented. Once the CAD modeling team resolved the identified issues in the models, the data set was designated as the reference set. Using the multi-CAD PMI validation technology, the specialist automatically compared each model from the other three CAD systems to the reference model.

Each discrepancy between the PMI in a model pair was compared with the test case to determine which model was inconsistent. Then, interactive CAD system queries were used to determine whether the discrepancy was due to measurement error in the validation tool or a difference in the test model. The validation software vendor resolved measurement errors while the CAD modeling team resolved model discrepancies within the limitations of the CAD system.

After several iterations of model refinement and verification, the outstanding discrepancies were documented as system limitations and the test models were released to the CAD software vendor representatives in the CAx Implementor Forum (CAx-IF) [26] for review. The CAD software vendors provided additional feedback to resolve any outstanding modeling issues. 


\section{PMI Modeling Capability Results}

The testing methodology was used to determine whether the representation and presentation of each PMI element (i.e., annotation, coordinate system, supplemental geometry entity, saved view) in each test model were well defined. The PMI element counts for this representative data set are shown in Table 3.

Table 3: PMI element counts by type and test case

\begin{tabular}{|c|c|c|c|c|c|c|}
\hline \multirow[b]{2}{*}{ PMI Element } & \multicolumn{5}{|c|}{ Element Count per Combined Test Case } & \multirow[b]{2}{*}{ Tota } \\
\hline & 1 & 2 & 3 & 4 & 5 & \\
\hline Annotation & 19 & 40 & 28 & 20 & 20 & 127 \\
\hline Coordinate System & 1 & 4 & 3 & 3 & 1 & 12 \\
\hline Supplemental Geometry Entity & 0 & 2 & 0 & 1 & 3 & 6 \\
\hline Saved View & 1 & 3 & 1 & 1 & 2 & 8 \\
\hline Total: & 21 & 49 & 32 & 25 & 26 & 153 \\
\hline
\end{tabular}

All PMI elements with a representation limitation were counted, by element type, across all test models for each CAD system. These counts were used to calculate a "Representation Limitation" percentage using this formula:

$$
\text { Limitation Percentage }=100 \times \frac{\text { Limitation Count }}{\text { Element Count }}
$$

All PMI elements with only a presentation limitation were counted and likewise divided by the element count to produce a "Presentation Limitations Only" percentage. If an element had both a representation and a presentation limitation, it was included only in the representation percentage. If an element had two or more representation and/or presentation limitations, it was counted only once in the appropriate calculation. Elements with neither type of limitation were counted in a "No Limitations" percentage, thus:

$$
\text { No Limitations }=100 \%-(\text { Representation Limitations }+ \text { Presentation Limitations })
$$

These three modeling capability percentages for each CAD system are shown in Figure 12. The names of the CAD systems have been generalized to give the end-user community an overall summary of their capabilities without impugning any particular CAD vendor. The technical details have been shared separately with each CAD vendor so they know their opportunity for improvement in the MBE domain. 


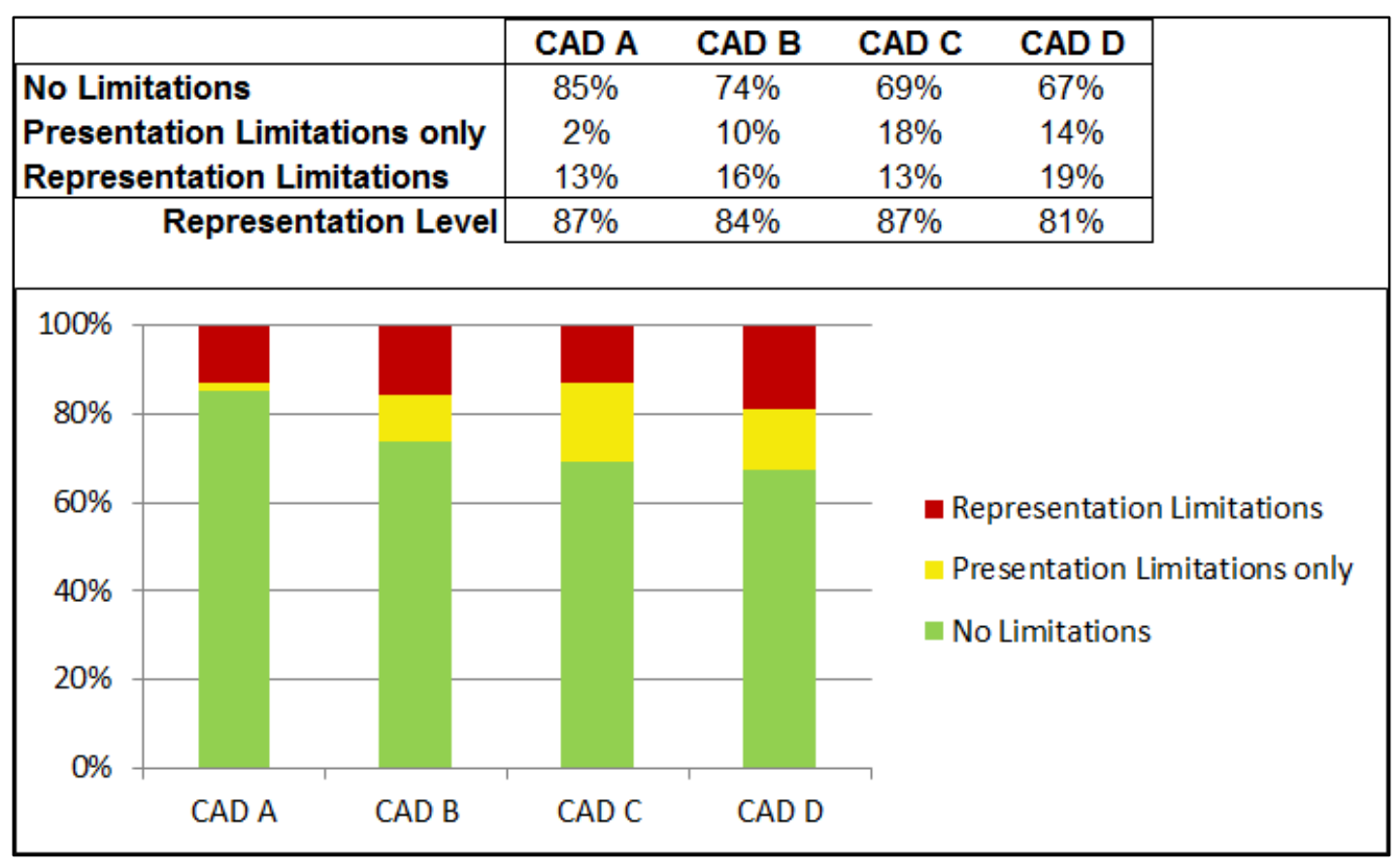

Figure 12: PMI modeling capability results by CAD system

In Figure 12, the "No Limitations" percentage can be interpreted as a measure of the capability of the CAD system to satisfy both the automated and visual consumption requirements of downstream MBE processes relative to the functional coverage of PMI constructs of this set of test cases. The "Representation Level" percentage, calculated as 100\% less the "Representation Limitations" percentage, indicates the CAD system’s ability to satisfy only automated consumption requirements.

The representation and presentation limitations for each CAD system were then subtotaled by characteristic and divided by the count of PMI elements of the type appropriate for that characteristic using this formula:

$$
\text { Verification Percentage }=100 \times \frac{\text { Element Count }- \text { Limitation Count }}{\text { Element Count }}
$$

For example, the count of annotation structure limitations for all models in each CAD system was divided by the count of annotations in the test case using the above formula. The verification percentages for each element type in each CAD system are shown in Table 4 and Table 5.

Table 4 reveals that all CAD systems failed to represent the expected structure of the specified coordinate systems. The remaining representation limitations were limited to annotations, although the specific percentages vary slightly. 
Table 4: PMI representation limitations by characteristic and CAD system

\begin{tabular}{|l|ccccc|}
\hline \multicolumn{1}{|c}{$\begin{array}{c}\text { Element } \\
\text { Representation Limitations }\end{array}$} & Count & CAD A & CAD B & CAD C & CAD D \\
\hline Annotation structure & 127 & $98 \%$ & $94 \%$ & $98 \%$ & $95 \%$ \\
Annotation parameters & 127 & $96 \%$ & $99 \%$ & $98 \%$ & $98 \%$ \\
Annotation geometry & 127 & $100 \%$ & $96 \%$ & $98 \%$ & $92 \%$ \\
Coordinate system structure & 12 & $\mathbf{0} \%$ & $\mathbf{0 \%}$ & $\mathbf{0 \%}$ & $\mathbf{0} \%$ \\
Coordinate system parameters & 12 & $100 \%$ & $100 \%$ & $100 \%$ & $100 \%$ \\
Supplemental geometry structure & 6 & $100 \%$ & $100 \%$ & $100 \%$ & $100 \%$ \\
Supplemental geometry parameters & 6 & $100 \%$ & $100 \%$ & $100 \%$ & $100 \%$ \\
\hline
\end{tabular}

Because the coordinate system structure limitations were consistent across all CAD systems, thus creating a uniform bias, it is useful to consider an adjustment to the overall statistics in Figure 12 that excludes all coordinate system limitations. Figure 13 shows these adjusted statistics.

\begin{tabular}{|c|c|c|c|c|c|}
\hline & CAD A & CAD B & CAD C & CAD D & \multirow{5}{*}{$\begin{array}{l}\text { Coordinate system } \\
\text { issues excluded }\end{array}$} \\
\hline No Limitations & $93 \%$ & $82 \%$ & $77 \%$ & $75 \%$ & \\
\hline Presentation Limitations only & $2 \%$ & $10 \%$ & $18 \%$ & $14 \%$ & \\
\hline Representation Limitations & $5 \%$ & $8 \%$ & $5 \%$ & $11 \%$ & \\
\hline Representation Level & $95 \%$ & $92 \%$ & $95 \%$ & $89 \%$ & \\
\hline
\end{tabular}

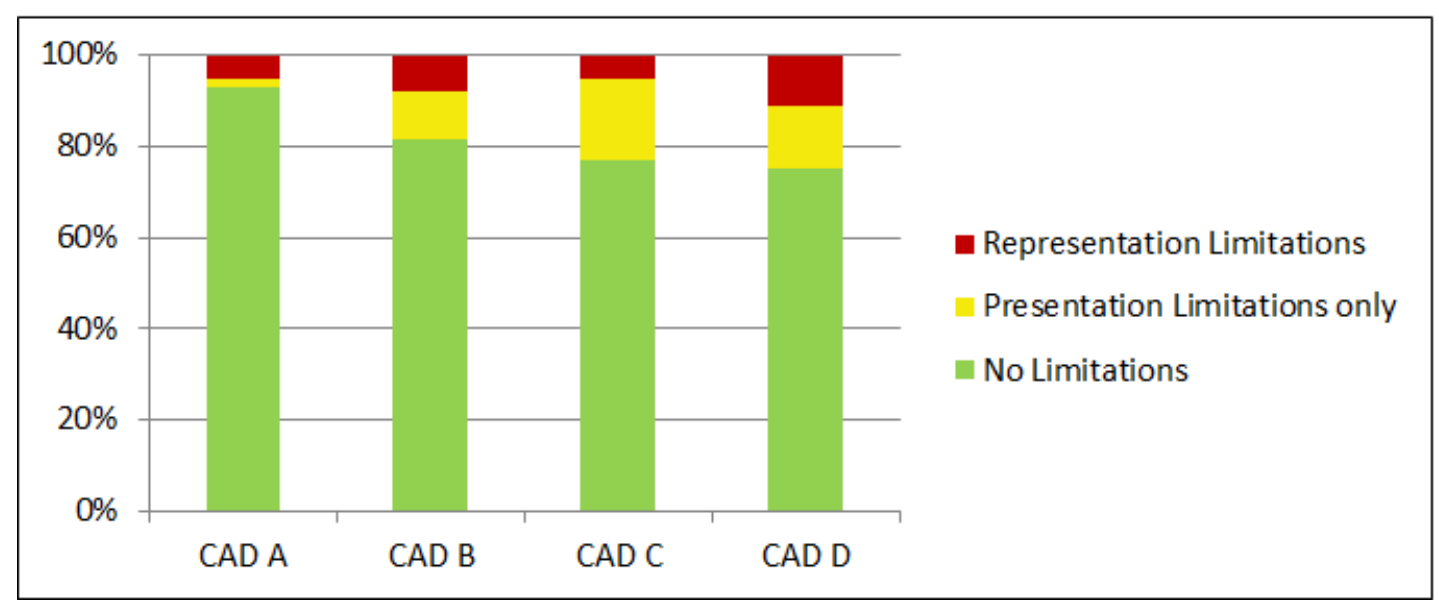

Figure 13: PMI modeling capability results by CAD system, excluding coordinate system structure limitations

Table 5 shows a much broader variation in the types of presentation limitations across CAD systems. Some of the systems were unable to adequately present coordinate system and saved view characteristics, which accounts for their larger overall "Presentation Limitations only" percentages relative to the other systems shown in Figure 13. 
Table 5: PMI presentation limitations by characteristic and CAD system

\begin{tabular}{|l|ccccc|}
\hline & Element & & & & \\
Presentation Limitations & Count & CAD A & CAD B & CAD C & CAD D \\
\hline Annotation visibility & 127 & $100 \%$ & $99 \%$ & $96 \%$ & $99 \%$ \\
Annotation color & 127 & $100 \%$ & $100 \%$ & $100 \%$ & $100 \%$ \\
Annotation name & 127 & $100 \%$ & $100 \%$ & $100 \%$ & $100 \%$ \\
Annotation layout & 127 & $98 \%$ & $96 \%$ & $93 \%$ & $98 \%$ \\
Annotation location & 127 & $99 \%$ & $100 \%$ & $94 \%$ & $98 \%$ \\
Annotation orientation & 127 & $100 \%$ & $100 \%$ & $98 \%$ & $99 \%$ \\
Annotation lines & 127 & $100 \%$ & $97 \%$ & $96 \%$ & $99 \%$ \\
Annotation text & 127 & $98 \%$ & $\mathbf{8 9} \%$ & $100 \%$ & $96 \%$ \\
Coordinate system visibility & 12 & $100 \%$ & $100 \%$ & $100 \%$ & $\mathbf{6 7 \%}$ \\
Coordinate system color & 12 & $100 \%$ & $100 \%$ & $100 \%$ & $100 \%$ \\
Coordinate system name & 12 & $100 \%$ & $100 \%$ & $\mathbf{8 3} \%$ & $100 \%$ \\
Coordinate system text & 12 & $100 \%$ & $100 \%$ & $100 \%$ & $\mathbf{6 7 \%}$ \\
Supplemental geometry visibility & 6 & $100 \%$ & $100 \%$ & $100 \%$ & $\mathbf{0 \%}$ \\
Supplemental geometry color & 6 & $100 \%$ & $100 \%$ & $100 \%$ & $\mathbf{1 0 0 \%}$ \\
Saved view structure & 8 & $100 \%$ & $100 \%$ & $100 \%$ & $\mathbf{0 \%}$ \\
Saved view name & 8 & $100 \%$ & $100 \%$ & $100 \%$ & $100 \%$ \\
Saved view frustum & 8 & $100 \%$ & $100 \%$ & $100 \%$ & $\mathbf{0 \%}$ \\
\hline
\end{tabular}




\subsection{Representation Limitations}

For each characteristic, there were often multiple types of limitations. Appendix D shows one example of each type of PMI representation limitation. The graphics in the appendices have been generalized to avoid identifying the specific CAD system involved. Figure 14 shows one example from Appendix D. Table 7 tabulates the count of representation limitations by characteristic and type across all CAD systems. Table 6 explains the PMI entity abbreviations used in Table 7.

\section{Annotation Parameters: Representation Limitation FCF parameter defined with encoded text}

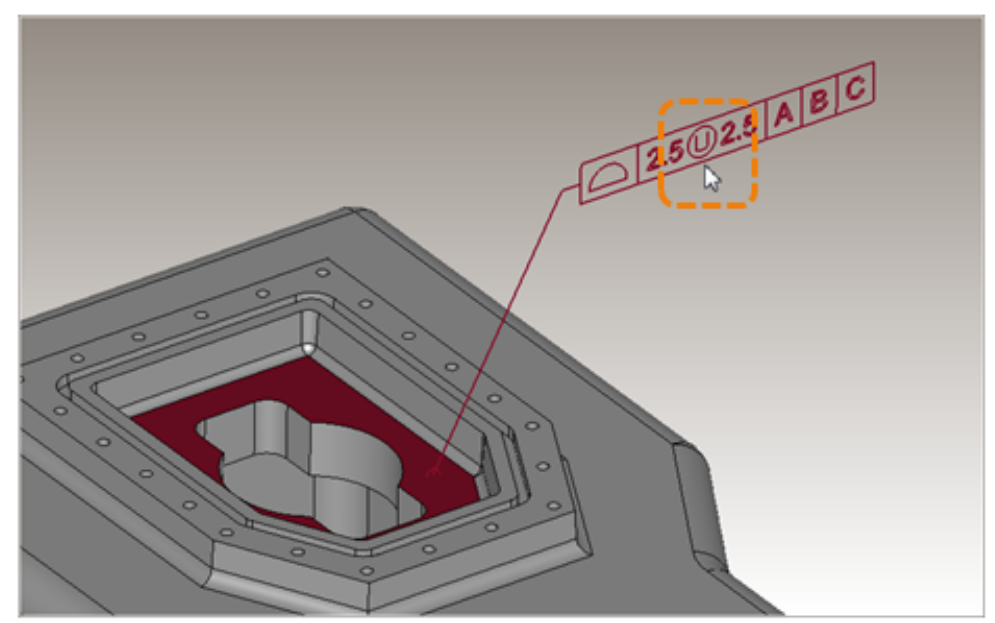

The unequally disposed modifier in this feature control frame is defined as a text symbol and not as a named parameter.

Figure 14: Example of a representation limitation

Table 6: PMI entity abbreviations

\begin{tabular}{|cl|}
\hline Abbrev & Definition \\
\hline AN & Annotation \\
CS & Coordinate system \\
DFS & Datum feature symbol \\
DIM & Dimension \\
DRF & Datum reference frame \\
DTS & Datum target symbol \\
FCF & Feature control frame \\
PG & Product geometry \\
SG & Supplemental geometry \\
VW & View \\
\hline
\end{tabular}


Table 7: Representation limitation counts by characteristic and type

\begin{tabular}{|lr|}
\hline Representation Limitations & $\mathbf{9 6}$ \\
$\boxminus$ Annotation structure & 19 \\
Countersink diameter DIM not defined & 1 \\
DIM defined as part of DTS & 4 \\
FCF extension line defined as separate DIM & 9 \\
FCF projected tolerance zone defined as separate DIM & 1 \\
FCF text defined as separate note & 3 \\
Threaded hole depth DIM not defined & 1 \\
$\boxminus$ Annotation parameters & $\mathbf{1 1}$ \\
DIM origin not defined & 1 \\
DIM parameter defined with encoded text & 3 \\
FCF between-basis defined with encoded text & 4 \\
FCF parameter defined with encoded text & 3 \\
$\boxminus$ Annotation geometry & 18 \\
DIM associated with extra face & 1 \\
DIM not associated with complete set of faces & 4 \\
DTS associated with extra face & 1 \\
DTS not associated with face & 1 \\
DTS not associated with SG point & 3 \\
FCF associated with extra face & 5 \\
FCF not associated with SG curve & 3 \\
$\boxminus$ Coordinate system structure & $\mathbf{4 8}$ \\
CS not linked to FCF DRF & 48 \\
\hline
\end{tabular}




\subsection{Presentation Limitations}

Appendix E shows one example of each type of presentation limitation. Figure 15 shows one example from Appendix E. Table 8 tabulates the count of representation limitations by characteristic and type across all CAD systems. Table 6 explains the PMI entity abbreviations used in Table 8.

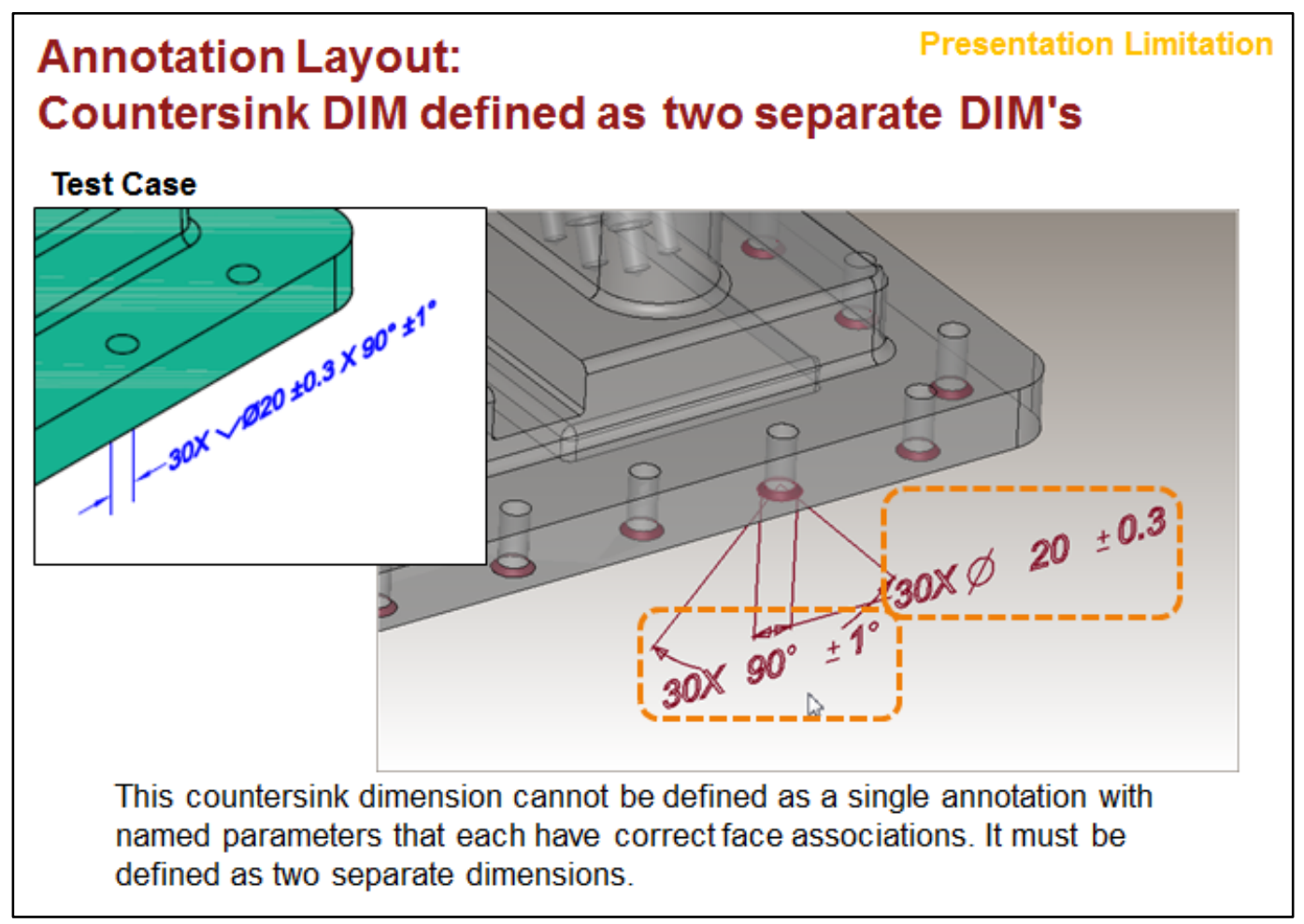

Figure 15: Example of a presentation limitation 
Table 8: Presentation limitation counts by characteristic and type

\begin{tabular}{|c|c|}
\hline$\Theta$ Presentation Limitations & 108 \\
\hline$\ominus$ Annotation visibility & 7 \\
\hline DFS is extraneous when DTS is defined & 2 \\
\hline DFS not visible in specified view & 1 \\
\hline DIM not visible in specified view & 1 \\
\hline DTS visible in wrong view & 3 \\
\hline$\boxminus$ Annotation layout & 20 \\
\hline Counterbore DIM defined as two separate DIM's & 4 \\
\hline Countersink DIM defined as two separate DIM's & 4 \\
\hline DIM limits displayed in reversed order & 1 \\
\hline DIM limits not displayed horizontally & 2 \\
\hline DTS target area diameter defined as separate DIM & 1 \\
\hline FCF text displayed above rather than below & 2 \\
\hline FCF text displayed on right rather than below & 3 \\
\hline Threaded hole DIM defined as two separate DIM's & 3 \\
\hline$\boxminus$ Annotation location & 12 \\
\hline DFS not attached to FCF & 8 \\
\hline DFS overlaps DIM graphics & 1 \\
\hline DFS partially buried in solid & 1 \\
\hline FCF partially buried in solid & 2 \\
\hline$\ominus$ Annotation orientation & 4 \\
\hline DIM text orientation is wrong & 1 \\
\hline DTS text is backwards in this view & 3 \\
\hline$\Theta$ Annotation lines & 10 \\
\hline DFS has no extension line & 10 \\
\hline$\ominus$ Annotation text & 22 \\
\hline DIM has extraneous space & 11 \\
\hline DTS text is extraneous & 2 \\
\hline FCF missing note text & 2 \\
\hline FCF missing projected tolerance zone length & 1 \\
\hline FCF text is extraneous & 6 \\
\hline$\Theta$ Coordinate system visibility & 4 \\
\hline CS visible in wrong view & 4 \\
\hline$\Theta$ Coordinate system name & 2 \\
\hline CS name not same as DRF & 2 \\
\hline$\Theta$ Coordinate system text & 4 \\
\hline CS name displayed with extra large text & 4 \\
\hline$\boxminus$ Supplemental geometry visibility & 7 \\
\hline SG curve visible in wrong view & 2 \\
\hline SG point visible in wrong view & 5 \\
\hline$\Theta$ Saved view structure & 8 \\
\hline View cannot contain annotations on different planes & 8 \\
\hline$\Theta$ Saved view frustum & 8 \\
\hline View camera position not defined & 8 \\
\hline
\end{tabular}




\subsection{Style Differences}

In some cases, the representation and presentation for a PMI element were determined by the expert reviewers to be correct yet different between the CAD systems. These variations were categorized as style differences and not included in the representation or presentation limitation calculations. Appendix $\mathrm{F}$ documents one example of each type of style difference that was ignored. Figure 16 shows an example from Appendix F. Table 9 tabulates the count of style differences by characteristic and type across all systems. Table 6 explains the PMI entity abbreviations used in Table 9.

\section{Annotation Structure: \\ Style Difference DTS requires DFS to be defined}

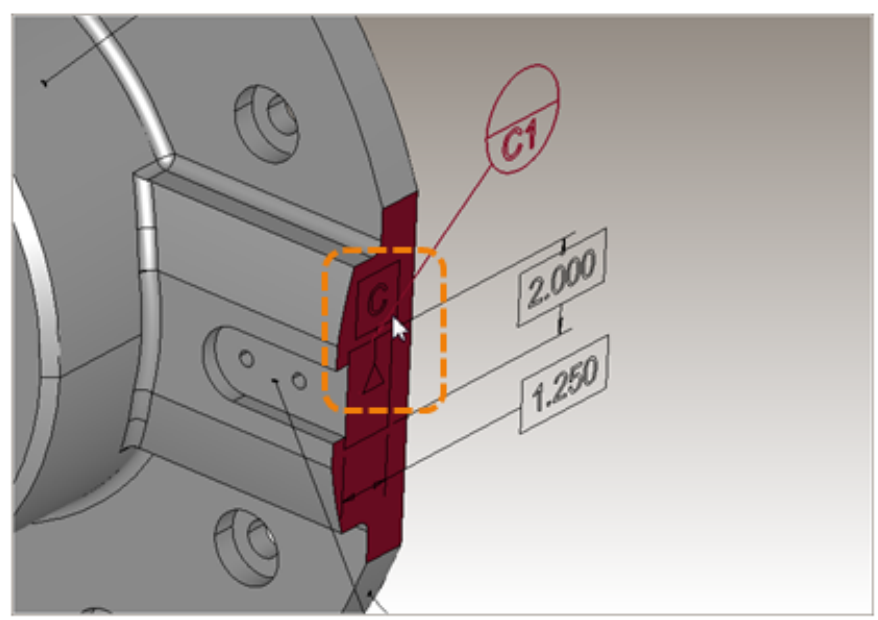

The system requires a datum feature symbol to be defined whenever a datum target symbol is defined.

Figure 16: Example of a style difference 
Table 9: Style difference counts by characteristic and type

\begin{tabular}{|lr|}
\hline$\Theta$ Style Differences & $\mathbf{4 8}$ \\
$\Theta$ Product geometry parameters & 1 \\
Threaded hole diameter different than other systems & 1 \\
$\boxminus$ Annotation structure & 19 \\
DTS requires DFS to be defined & 18 \\
FCF requires DFS to be defined & 1 \\
$\boxminus$ Annotation geometry & $\mathbf{1 1}$ \\
DFS edge association is extraneous & 2 \\
DIM edge association is extraneous & 9 \\
$\Theta$ Supplemental geometry structure & 17 \\
DTS target area is non-solid surface on solid face & 6 \\
DTS target area is subdivided solid face & 1 \\
DTS target area is wireframe region on solid face & 5 \\
FCF limited area definition inconsistent with target area & 1 \\
FCF limited area is non-solid surface on solid face & 3 \\
FCF limited area is subdivided solid face & 1 \\
\hline
\end{tabular}

\subsection{PMI Verification Challenges}

A challenging construct is the representation of extension lines for datum feature symbols and feature control frames. In some CAD systems, this construct is represented as dimension entities that are separate from the attached annotation, as shown in Figure 17. These extra annotations introduce parameters (nominal value and limits) that must be ignored during verification.

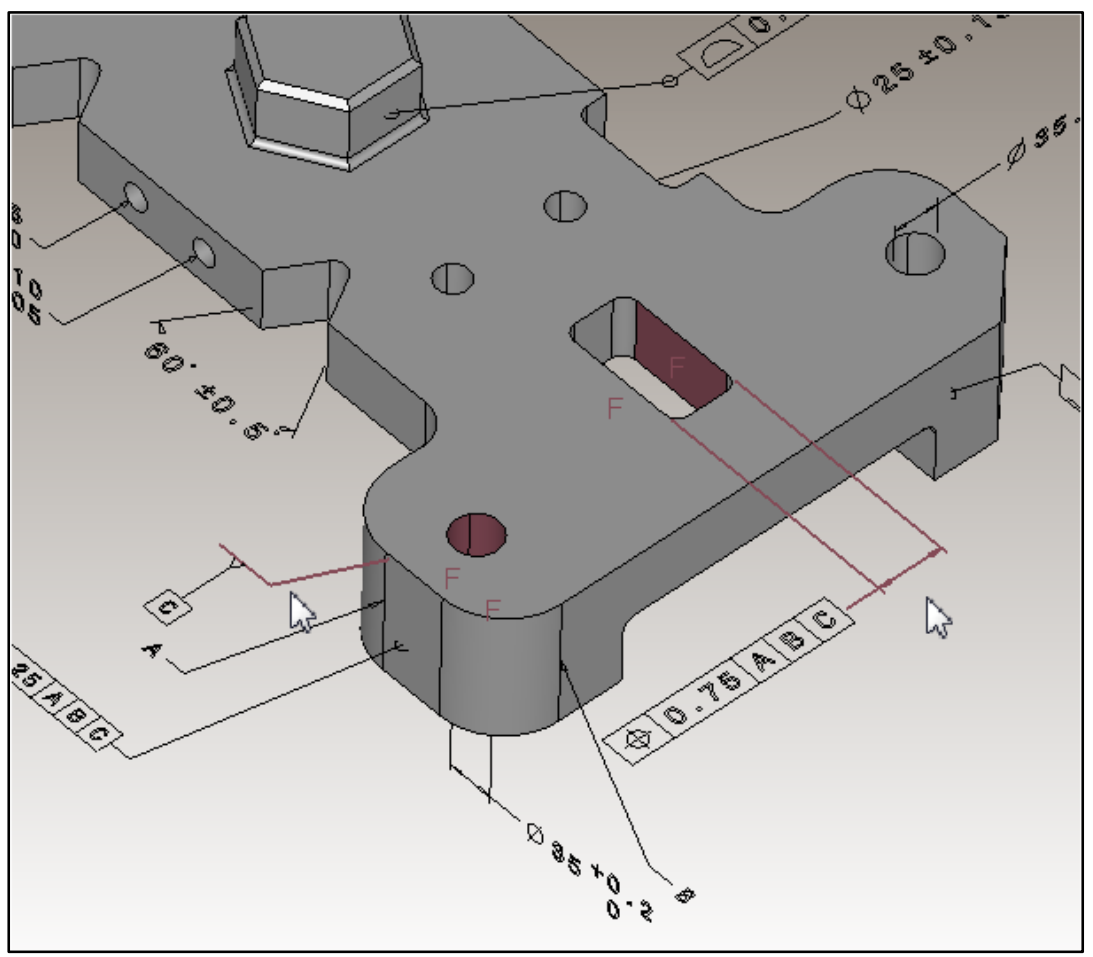

Figure 17: Extension lines represented as separate dimension annotations 
Another challenging construct is the representation of threaded holes shown in Figure 18. The diameter of the simple hole in the solid model may be different for the same type of threaded hole in various CAD systems. Some systems use differing supplemental geometry, such as wireframe curves or non-solid surfaces, to represent the hole thread depth while others use no supplemental geometry.

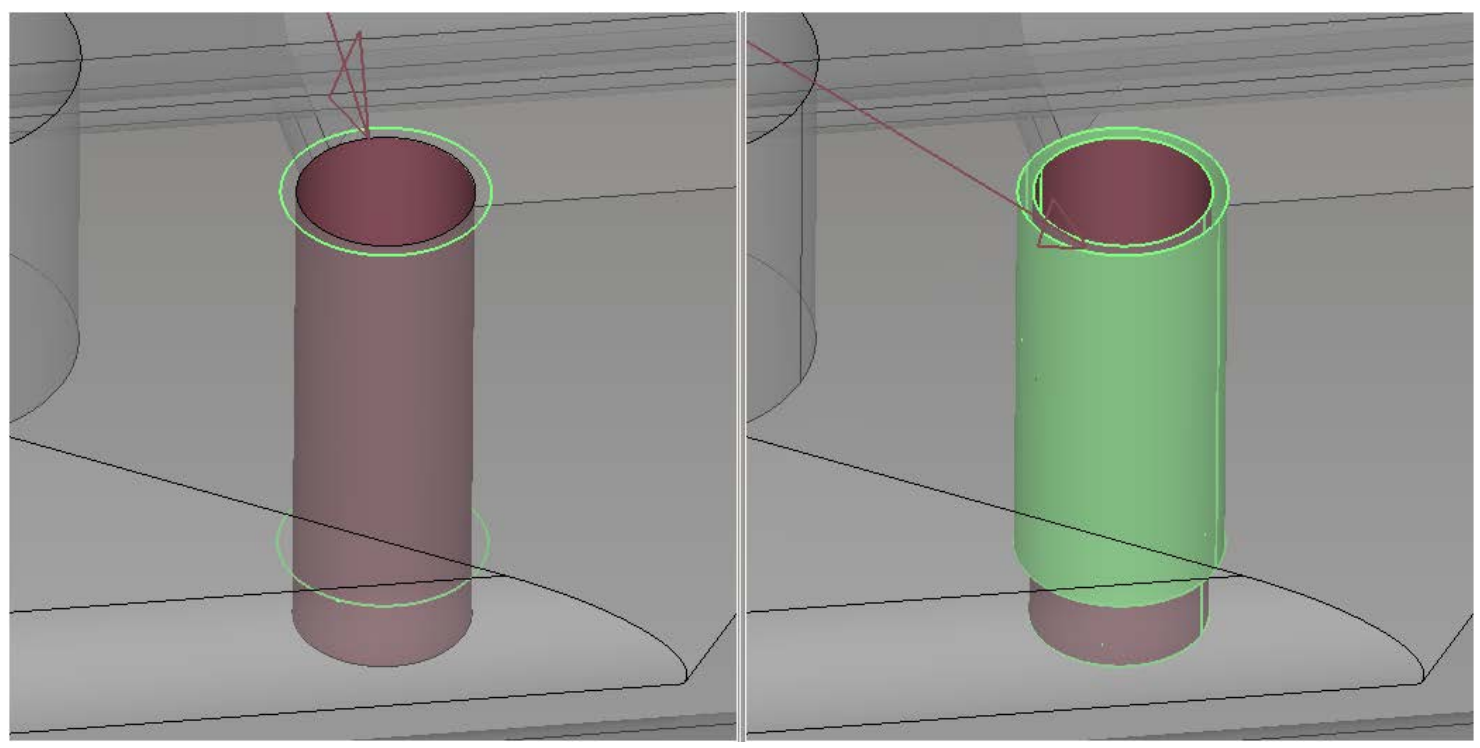

Figure 18: Different threaded hole supplemental geometry representations

Finally, when a PMI construct is specified with a limited area, such as a datum target or geometric tolerance, the portion of the product shapes that is within the target area is represented differently. Some CAD systems define a non-solid surface overlaid on the solid while others subdivide the portion of the solid face into a separate face shown in Figure 19. Still others indicate the area with a region defined by wireframe geometry. These modeling differences create significant variability that must be accounted for during annotation matching and comparison.
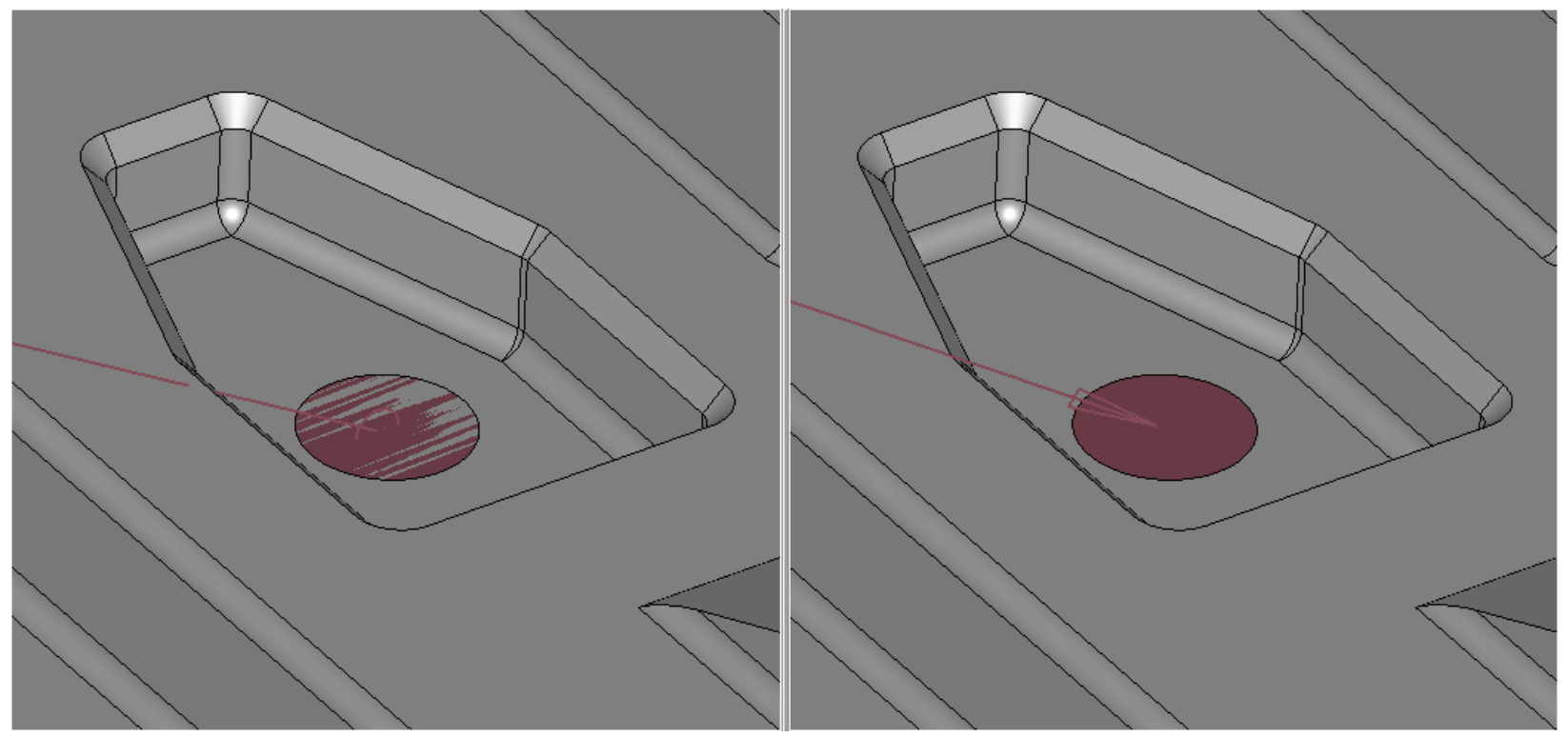

Figure 19: Different target area representations 


\section{Discussion}

Using a formal methodology, implemented with advanced verification and validation technology, the MBE modeling capability of four leading CAD systems was quantified relative to the PMI requirements captured in five combined test cases.

The four CAD systems, which were tested at 2013 release levels, are roughly equivalent in their PMI representation modeling capability (between $81 \%$ and $87 \%$ ) for the functionality within scope of this assessment (between $89 \%$ and 95\% when adjusted for the consistent coordinate system limitation). Their capability measurements vary much more (between 67\% and 85\%) when both automated and visual consumption requirements are considered.

The specific PMI representation and presentation system limitations identified by this assessment have been clearly documented and communicated to the CAD vendors.

The specific test of the PMI capabilities in CAD systems documented in this report is a snapshot in time. Specific test cases were developed using particular versions of the ASME Y14 tolerancing standards and PMI constructs. The test cases were modeled in particular versions of four CAD systems with a specific modeling methodology to give precedence to PMI representation over PMI presentation. The CAD models were compared to each other with a particular version of CAD validation software. Results for PMI representation and presentation capabilities were reported based on four categories of PMI elements: annotations, coordinate systems, supplemental geometry, and saved views.

For a company that is transitioning from 2D drawings to 3D models to implement model-based design, this report can be used to identify the characteristics of PMI representation and presentation and the capabilities of CAD software that are important to achieve an MBD workflow. The test cases may or may not be representative of the types of PMI that might be typically used. The versions of the CAD systems and tolerancing standards might be newer or older than what a company requires. However, the report clearly identifies a wide variety of PMI representation and presentation issues that can be used to evaluate CAD software that is used in an MBD environment. 


\section{References}

[1] Model Based Enterprise - Exploring the Digital Tapestry, http://www.model-basedenterprise.org/.

[2] J. Lubell, K. Chen, J. Horst, S. Frechette, and P. Huang, "Model Based Enterprise / Technical Data Package Summit Report," NIST Technical Note 1753, 2012.

[3] J. Lubell, S. P. Frechette, R. R. Lipman, F. M. Proctor, J. A. Horst, M. Carlisle, and P. J. Huang, "Model-Based Enterprise Summit Report," National Institute of Standards and Technology, NIST Technical Note 1820, 2013.

[4] Model-Based Enterprise Summit 2013, National Institute of Standards and Technology, http://www.nist.gov/el/msid/mbe_2013_presentations.cfm.

[5] Model-Based Enterprise Summit 2014, National Institute of Standards and Technology, http://www.nist.gov/el/msid/mbe2014presentations.cfm.

[6] V. Quintana, L. Rivest, R. Pellerin, F. Venne, and F. Kheddouci, "Will Model-based Definition replace engineering drawings throughout the product lifecycle? A global perspective from aerospace industry," Computers in Industry, vol. 61, pp. 497-508, 2010.

[7] M. Alemanni, F. Destefanis, and E. Vezzetti, "Model-based definition design in the product lifecycle management scenario," The International Journal of Advanced Manufacturing Technology, vol. 52, pp. 1-14, 2011.

[8] ISO 10303-1:1994, "Industrial automation systems and integration - Product data representation and exchange - Part 1: Overview and fundamental principles," International Organization for Standardization, Geneva, Switzerland.

[9] M. J. Pratt, "Introduction to ISO 10303 - the STEP standard for product data exchange," Journal of Computing and Information Science in Engineering, vol. 1, pp. 102-103, 2001.

[10] STEP Application Handbook, ISO 10303, Version 3, SCRA, 2006, https://pdesinc.org/downloadable_files/STEPapplicationhandbook63006BF.pdf.

[11] JT Open, Siemens, http://www.plm.automation.siemens.com/en_us/products/open/jtopen/.

[12] JT Implementor Forum, ProSTEP iViP, http://www.prostep.org/en/projects/jt-implementorforum.html.

[13] ISO 14306:2012, "Industrial automation systems and integration - JT file format specification for 3D visualization," International Organization for Standardization, Geneva, Switzerland.

[14] 3D PDF Consortium, http://www.3dpdfconsortium.org/.

[15] ISO 24517-1:2008, "Document management -- Engineering document format using PDF -- Part 1: Use of PDF 1.6 (PDF/E-1)," International Organization for Standardization, Geneva, Switzerland.

[16] ISO 14739-1:2014, "Document management -- 3D use of Product Representation Compact (PRC) format -- Part 1: PRC 10001," International Organization for Standardization, Geneva, Switzerland.

[17] ISO/PAS 26183:2006, "SASIG Product data quality guidelines for the global automotive industry," International Organization for Standardization, Geneva, Switzerland.

[18] PDES, Inc. - Advancing the Digital Enterprise, https://pdesinc.org/.

[19] ProSTEP iViP Association, http://prostep.org/.

[20] LOTAR - Long Term Archiving and Retrieval, http://www.lotar-international.org/.

[21] Development of a Convergent Modular STEP Application Protocol Based on AP 203 and AP 214: STEP AP 242 - Managed Model Based 3D Engineering, ASD Strategic Standardization Group, 2009, http://www.ap242.org/.

[22] ISO 10303-242:2014, "Industrial automation systems and integration - Product data representation and exchange - Part 242: Application protocol: Managed Model-based 3D Engineering," International Organization for Standardization, Geneva, Switzerland.

[23] ASD Strategic Standardization Group - Long Term Archiving and Retrieval - LOTAR, http://www.asd-ssg.org/lotar. 
[24] MIL-STD-31000A, "DoD Standard Practice: Technical Data Packages," U.S. Department of Defense, 2013.

[25] S. P. Frechette, A. T. Jones, and B. R. Fischer, "Strategy for Testing Conformance to Geometric Dimensioning \& Tolerancing Standards," Procedia CIRP, vol. 10, pp. 211-215, 2013.

[26] CAx Implementor Forum, http://www.cax-if.org/.

[27] D. Cheney and B. Fischer, "Measuring the PMI Modeling Capability in CAD Systems: Report 2 Test Case Validation," National Institute of Standards and Technology, NIST-GCR 15-998, 2015.

[28] D. Cheney and B. Fischer, "Measuring the PMI Modeling Capability in CAD Systems: Report 3 Fully-Toleranced Test Case Verification," National Institute of Standards and Technology, NISTGCR 15-999, 2015.

[29] ASME Y14.5-1994, "Dimensioning and Tolerancing - Engineering Drawing and Related Documentation Practices," American Society of Mechanical Engineers, 1994, New York.

[30] ASME Y14.41-2003, "Digital Product Definition Data Practices - Engineering Drawing and Related Documentation Practices," American Society of Mechanical Engineers, 2003, New York.

[31] R. Lipman and J. Lubell, "Conformance checking of PMI representation in CAD model STEP data exchange files," Computer-Aided Design, vol. 66, pp. 14-23, 2015.

[32] CATIA, Dassault Systemes, http://www.3ds.com/products-services/catia/.

[33] Creo, PTC, http://www.ptc.com/product/creo.

[34] NX, Siemens, www.plm.automation.siemens.com/en us/products/nx/.

[35] SOLIDWORKS, Dassault Systemes, http://www.solidworks.com/.

[36] CADIQ, ITI TranscenData, http://www.transcendata.com/products/cadiq/. 


\section{Appendix A: PMI Constructs}

\begin{tabular}{|c|c|c|c|c|}
\hline $\begin{array}{c}\text { PMI } \\
\text { Construc }\end{array}$ & СТC & Construct Description & Units & Construct Specification \\
\hline 1 & 1 & Dimension with Equal-Bilateral Tolerance: Feature of Size & $\mathrm{mm}$ & $\varnothing 25 \pm 0.15$ \\
\hline 2 & 1 & Dimension with Unequal-Bilateral Tolerance: Feature of Size & $\mathrm{mm}$ & $\begin{array}{l}\varnothing 20+0.05 /-0.10 \\
\varnothing 20+0.10 /-0.05\end{array}$ \\
\hline 3 & 1 & Dimension with Unilateral Tolerance: Feature of Size & $\mathrm{mm}$ & $\begin{array}{l}\varnothing 350 /-0.2 \\
\varnothing 35+0.2 / 0\end{array}$ \\
\hline 4 & 1 & Angular Dimension with Equal-Bilateral Tolerance: Simple & $\mathrm{mm}$ & $60^{\circ} \pm 0.5^{\circ}$ \\
\hline 5 & 4 & Directly-toleranced dimension with nX (quantity) & $\mathrm{mm}$ & $30 \mathrm{X} \emptyset 14 \pm 0.1$ \\
\hline 6 & 3 & Directly-Toleranced Dimension with Dimension Origin Symbol & inch & $4 X .82 \pm .06$ (origin) \\
\hline 7 & 1 & $\begin{array}{l}\text { Symbol: All Around (Applied with a Leader-Directed Profile } \\
\text { Tolerance) }\end{array}$ & $\mathrm{mm}$ & $\bigcirc 0.5 \mid \mathrm{A}$ All Around \\
\hline 8 & 1 & $\begin{array}{l}\text { Dimension: Limit - Vertical (Stacked) and Horizontal with } \\
\text { Diameter Symbol: Feature of Size }\end{array}$ & $\mathrm{mm}$ & $\begin{array}{l}\varnothing 34.8-35.2 \\
\varnothing 35.2 / 34.8\end{array}$ \\
\hline 9 & 4 & $\begin{array}{l}\text { Symbol: Counterbore; Symbol: Depth - Single-Line } \\
\text { Specification of Two Dimensions and Tolerances - Complex }\end{array}$ & $\mathrm{mm}$ & $4 X \sqcup \sqcup \varnothing 20 \pm 0.2 \rrbracket 20 \pm 0.2$ \\
\hline 10 & 4 & $\begin{array}{l}\text { Symbol: Countersink - Single-Line Specification of Two } \\
\text { Dimensions and Tolerances - Complex }\end{array}$ & $\mathrm{mm}$ & $30 \mathrm{X} \vee \varnothing 20 \pm 0.3 \times 90^{\circ} \pm 1^{\circ}$ \\
\hline 11 & 5 & $\begin{array}{l}\text { Directly-Toleranced Dimension with Statistical Tolerancing } \\
\text { Symbol }\end{array}$ & inch & $5.000 \pm .008$ ST \\
\hline 12 & 4 & Basic dimension & $\mathrm{mm}$ & $\begin{array}{l}75 \text { basic } \\
\varnothing 10 \text { basic }\end{array}$ \\
\hline 13 & 3 & Reference Dimension, Simple & inch & $(.750)$ \\
\hline 14 & 3 & $\begin{array}{l}\text { Single Segment Feature Control Frame, Simple - Attached to } \\
\text { Size Dimension \& Tolerance }\end{array}$ & inch & $\begin{array}{l}\phi \varnothing .06|\mathrm{D}| \mathrm{B} \mid \mathrm{C} \\
\perp \varnothing .01 \mid \mathrm{E}\end{array}$ \\
\hline 15 & 4 & $\begin{array}{l}\text { Composite Feature Control Frame - } 2 \text { Segments - Leader } \\
\text { Directed - with String Grouping Mechanism }\end{array}$ & $\mathrm{mm}$ & $\begin{array}{c}\bigcirc 2|\mathrm{D}| \mathrm{G} \mid \mathrm{H} \\
0.2 \mid \mathrm{D} \\
2 \text { SURFACES }\end{array}$ \\
\hline 16 & 4 & $\begin{array}{l}\text { Composite Feature Control Frame - } 2 \text { segments - Attached to } \\
\text { Directly-Toleranced Size Dimension }\end{array}$ & $\mathrm{mm}$ & $\begin{array}{c}6 \mathrm{X} ø 6.65 \pm 0.12 \\
\emptyset_{\emptyset 1.5|\mathrm{D}| \mathrm{E} \mid \mathrm{F}} \\
\varnothing 0.3|\mathrm{D}| \mathrm{E}\end{array}$ \\
\hline 17 & 1 & Feature Control Frame Directed to Surface - Flatness & $\mathrm{mm}$ & $\square 0.2$ \\
\hline 18 & 5 & $\begin{array}{l}\text { Feature Control Frame Directed to Surface - Straightness } \\
\text { with Represented Line Element }\end{array}$ & inch & -.005 \\
\hline 19 & 5 & Feature Control Frame Directed to Surface - Circularity & inch & О.002 \\
\hline 20 & 3 & Feature Control Frame Directed to Surface - Angularity & inch & $\angle .04 \mid \mathrm{A}$ \\
\hline
\end{tabular}




\begin{tabular}{|c|c|c|c|c|}
\hline $\begin{array}{c}\text { PMI } \\
\text { Construc }\end{array}$ & СTC & Construct Description & Units & Construct Specification \\
\hline 21 & 1 & Feature Control Frame Directed to Surface - Perpendicularity & $\mathrm{mm}$ & $\perp 1.5 \mid \mathrm{A}$ \\
\hline 22 & 4 & Feature Control Frame Directed to Surface - Position & $\mathrm{mm}$ & Ф $\varnothing 0.35|\mathrm{~A}| \mathrm{B} \mid \mathrm{C}$ \\
\hline 23 & 5 & Feature Control Frame Directed to Surface - Concentricity & inch & (C) $\varnothing .030 \mid \mathrm{B}$ \\
\hline 24 & 5 & Feature Control Frame Directed to Surface - Circular Runout & inch & $\nearrow_{.025 \mid \mathrm{A}-\mathrm{B}}$ \\
\hline 25 & 5 & Feature Control Frame Directed to Surface - Total Runout & inch & $\begin{array}{l}\not 8.002 \mid \mathrm{A} \\
\Delta 8.015 \mid \mathrm{B}\end{array}$ \\
\hline 26 & 2 & $\begin{array}{l}\text { Feature Control Frame Directed to Surface - Profile of a } \\
\text { Surface }\end{array}$ & $\mathrm{mm}$ & $\bigcirc 0.75|\mathrm{~A}| \mathrm{B} \mid \mathrm{C}$ \\
\hline 27 & 3 & Feature Control Frame with Unit-Basis Tolerance - Flatness & inch & $\square .005 / .25 \times .25$ \\
\hline 28 & 2 & Feature Control Frame with MMC Modifier & $\mathrm{mm}$ & $\varnothing 1.5(\mathrm{M})|\mathrm{A}| \mathrm{B} \mid \mathrm{C}$ \\
\hline 29 & 2 & Feature Control Frame with LMC Modifier & $\mathrm{mm}$ & $\phi \varnothing 1(\mathrm{D})|\mathrm{A}| \mathrm{B} \mid \mathrm{C}$ \\
\hline 30 & 4 & $\begin{array}{l}\text { Feature Control Frame with Projected Tolerance and } \\
\text { Projection Distance - ASME }\end{array}$ & $\mathrm{mm}$ & $\begin{array}{l}4 \mathrm{X} M 12 \times 1.75-6 \mathrm{H} \nabla 25 \pm 0.25 \\
\emptyset_{\emptyset 0.75 \odot 50|\mathrm{~A}| \mathrm{B} \mid \mathrm{C}}\end{array}$ \\
\hline 31 & 2 & $\begin{array}{l}\text { Feature Control Frame with ASME Modifiers - Unequally- } \\
\text { Disposed }\end{array}$ & $\mathrm{mm}$ & $\begin{array}{l}\bigcirc 2.5(\mathrm{U}) 0.5|\mathrm{~A}| \mathrm{B} \mid \mathrm{C} \\
\bigcirc 2.5(\mathrm{U}) 2.5|\mathrm{~A}| \mathrm{B} \mid \mathrm{C} \\
\bigcirc 2.5(\mathrm{U}) 0|\mathrm{~A}| \mathrm{B} \mid \mathrm{C}\end{array}$ \\
\hline 32 & 3 & $\begin{array}{l}\text { Unidirectional Positional Tolerancing - Parallel Plane } \\
\text { Tolerance Zone for Cylindrical Feature of Size }\end{array}$ & inch & Ф.03|D|B|C \\
\hline 33 & 1 & $\begin{array}{l}\text { Single Segment Feature Control Frame - Attached Directly to } \\
\text { Dimension Lines - No Dimension Value }\end{array}$ & $\mathrm{mm}$ & $\oplus_{0.75|\mathrm{~A}| \mathrm{B} \mid \mathrm{C}}$ \\
\hline 34 & 2 & $\begin{array}{l}\text { Feature Control Frame with MMB Modifiers for Datum Feature } \\
\text { References - ASME }\end{array}$ & $\mathrm{mm}$ & $\begin{array}{l}\bigcirc 0.5|\mathrm{D}| \mathrm{E}(\mathrm{M}) \mid \mathrm{F}(\mathrm{M}) \\
\bigcirc 0.5|\mathrm{D}| \mathrm{E}(\mathrm{M}) \mid \mathrm{F} \\
\bigcirc 0.5|\mathrm{D}| \mathrm{E} \mid \mathrm{F} M \\
\bigcirc 0.5|\mathrm{D}| \mathrm{E} \mid \mathrm{F}\end{array}$ \\
\hline 35 & 2 & $\begin{array}{l}\text { Feature Control Frame with LMB Modifiers for Datum Feature } \\
\text { References - ASME }\end{array}$ & $\mathrm{mm}$ & $\begin{array}{l}\bigcirc 0.8|\mathrm{G}| \mathrm{H}(\mathrm{L} \mid \mathrm{J} \\
\bigcirc 0.8|\mathrm{G}| \mathrm{H}(\mathrm{L} \mid \mathrm{J}(\mathrm{L}) \\
\bigcirc 0.8|\mathrm{G}| \mathrm{H} \mid \mathrm{J}(\mathrm{L}) \\
\bigcirc 0.8|\mathrm{G}| \mathrm{H} \mid \mathrm{J} \\
\text { DFS G } 4 \text { COPLANAR SURFACES }\end{array}$ \\
\hline
\end{tabular}




\begin{tabular}{|c|c|c|c|c|}
\hline $\begin{array}{c}\text { PMI } \\
\text { Construc }\end{array}$ & CTC & Construct Description & Units & Construct Specification \\
\hline 36 & 3 & Feature Control Frame with MMC and MMB Modifiers & inch & $\phi \varnothing .05(\mathrm{M})|\mathrm{A}| \mathrm{B} M \mid \mathrm{C}(\mathrm{M})$ \\
\hline 37 & 5 & Datum Feature symbol attached to Feature of Size & inch & A \\
\hline 38 & 5 & Datum Feature symbol attached to a Size Dimension & inch & $\begin{array}{l}\mathrm{B} \\
\varnothing 10.000 \pm .001\end{array}$ \\
\hline 39 & 3 & $\begin{array}{l}\text { Datum Feature Symbol Attached to a Leader-Directed } \\
\text { Feature Control Frame }\end{array}$ & inch & $\begin{array}{l}\bigcirc .012 \text { COPLANAR SURFACES + DFS A } \\
\bigcirc .03|\mathrm{~A}| \mathrm{B} \mid \mathrm{C}+\text { DFS D } \\
\bigcirc .06|\mathrm{~A}| \mathrm{B} \mid \mathrm{C}+\mathrm{DFS} E\end{array}$ \\
\hline 40 & 4 & $\begin{array}{l}\text { Datum Feature symbols for Primary, Secondary, Tertiary } \\
\text { attached to surfaces }\end{array}$ & $\mathrm{mm}$ & $\mathrm{D}, \mathrm{E}, \mathrm{F}, \mathrm{G}, \mathrm{H}$ \\
\hline 41 & 2 & $\begin{array}{l}\text { Datum Target Symbol and Target Area Symbol Applied to } \\
\text { Surface: Area Defined in Datum Target Symbol }(\varnothing)\end{array}$ & $\mathrm{mm}$ & $\varnothing 0.85$ Area K1 \\
\hline 42 & 5 & $\begin{array}{l}\text { Datum Target Symbol and Target Area Applied to Surface: } \\
\text { Area Defined on Surface (Rectangular) }\end{array}$ & inch & $\begin{array}{l}\text { C1, D1 } \\
2.000 \text { (basic), } 1.250 \text { (basic) }\end{array}$ \\
\hline 43 & 2 & $\begin{array}{l}\text { Set of Datum Target Symbols and Target Point Symbols } \\
\text { Applied to Surfaces }\end{array}$ & $\mathrm{mm}$ & $\begin{array}{l}A 1, A 2, A 3 \\
B 1, B 2, B 3, B 4 \\
C 1\end{array}$ \\
\hline 44 & 5 & Multiple Datum Feature & inch & $\begin{array}{l}\nearrow_{0.035 \mid A-B} \\
.025 \mid A-B\end{array}$ \\
\hline 45 & 3 & $\begin{array}{l}\text { Size Dimension with Feature Control Frame and STRING - } \\
\text { Applied } n X\end{array}$ & inch & $\begin{array}{l}4 X \varnothing .625 \pm .005 \\
\emptyset \varnothing .05(M)|D| B \mid C \\
\text { SWITCH MOUNTING LOCATIONS }\end{array}$ \\
\hline 46 & 3 & $\begin{array}{l}\text { Size Dimension with Feature Control Frame and Datum } \\
\text { Feature Symbol Attached }\end{array}$ & inch & $\begin{array}{l}\varnothing .438 \pm .005 \\
\perp \varnothing .01 \mid \mathrm{A}+\mathrm{DFS} \mathrm{B} \\
\varnothing .438 \pm .005 \\
\phi \varnothing .02|\mathrm{~A}| \mathrm{B}+\mathrm{DFS} \mathrm{C} \\
\varnothing 1.065 \pm .003 \\
\perp \varnothing .01 \mid \mathrm{E}+\mathrm{DFS} F\end{array}$ \\
\hline 47 & 2 & $\begin{array}{l}\text { Directional Geometric Tolerance with Represented Line } \\
\text { Element }\end{array}$ & $\mathrm{mm}$ & $\frown 0.25|\mathrm{~A}| \mathrm{B} \mid \mathrm{C}$ \\
\hline 48 & 1 & Profile Tolerance: Applied on a Between Basis & $\mathrm{mm}$ & $\begin{array}{l}\bigcirc 1.25|\mathrm{~A}| \mathrm{B} \mid \mathrm{C} \\
\mathrm{A} \leftarrow \rightarrow \mathrm{B}\end{array}$ \\
\hline 49 & 4 & $\begin{array}{l}\text { Profile Tolerance Applied to a Limited Area (Circular Area) - } \\
\text { Area Not Explicitly Dimensioned }\end{array}$ & $\mathrm{mm}$ & $\bigcirc 0.5|\mathrm{~A}| \mathrm{B} \mid \mathrm{C}$ \\
\hline 50 & 2 & $\begin{array}{l}\text { General Notes Invoking ASME Y14.5M-1994 and Y14.41-2003 } \\
\text { on Static Annotation Plane }\end{array}$ & $\mathrm{mm}$ & $\begin{array}{l}\text { Obtain dimensions from model... } \\
\text { Model geometry is basic ... } \\
\text { ASME Y14.41-2003 applies... } \\
\text { ASME Y14.5M-1994 applies... }\end{array}$ \\
\hline
\end{tabular}




\section{Appendix B: Combined Test Case Drawings}
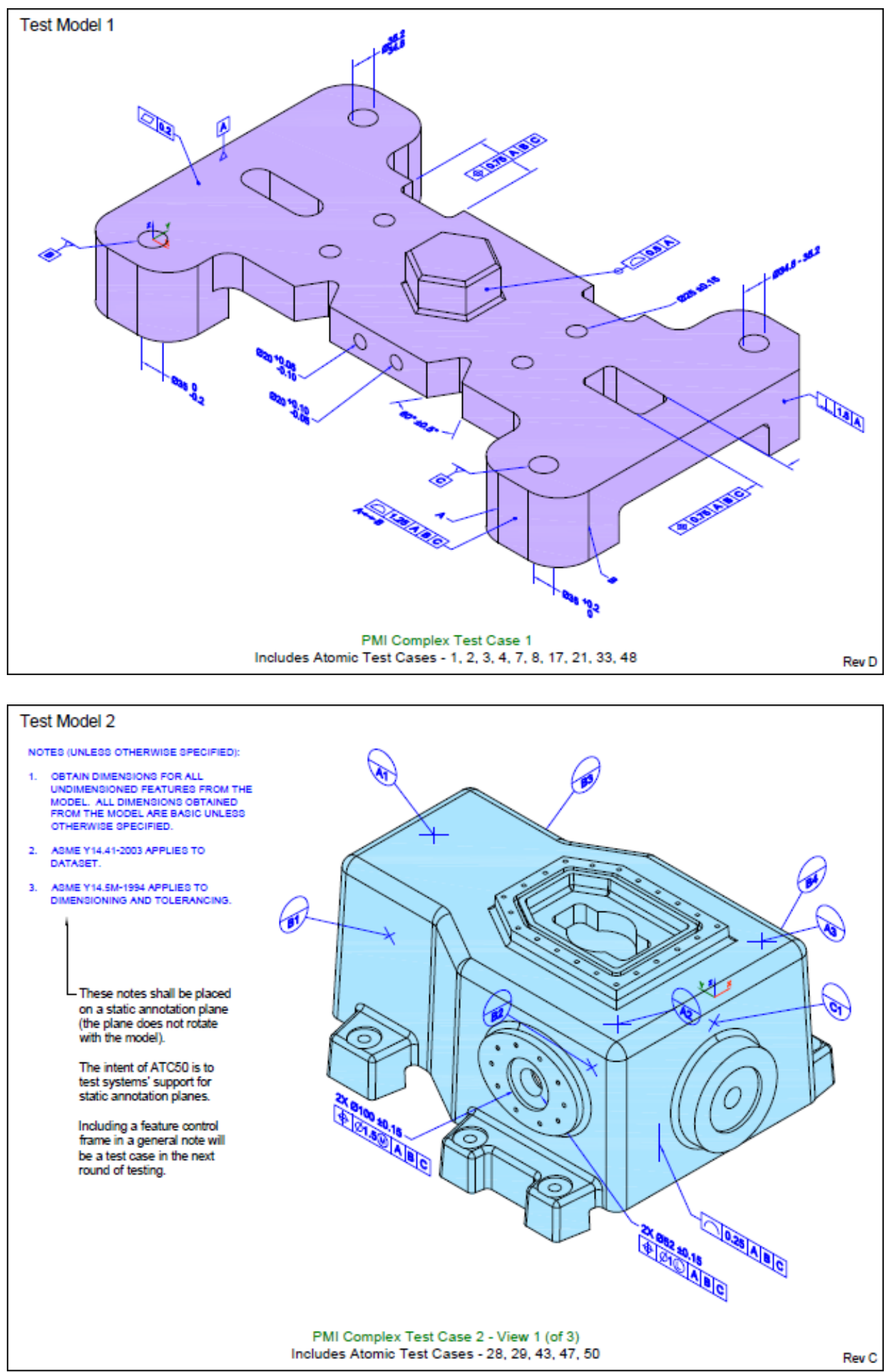

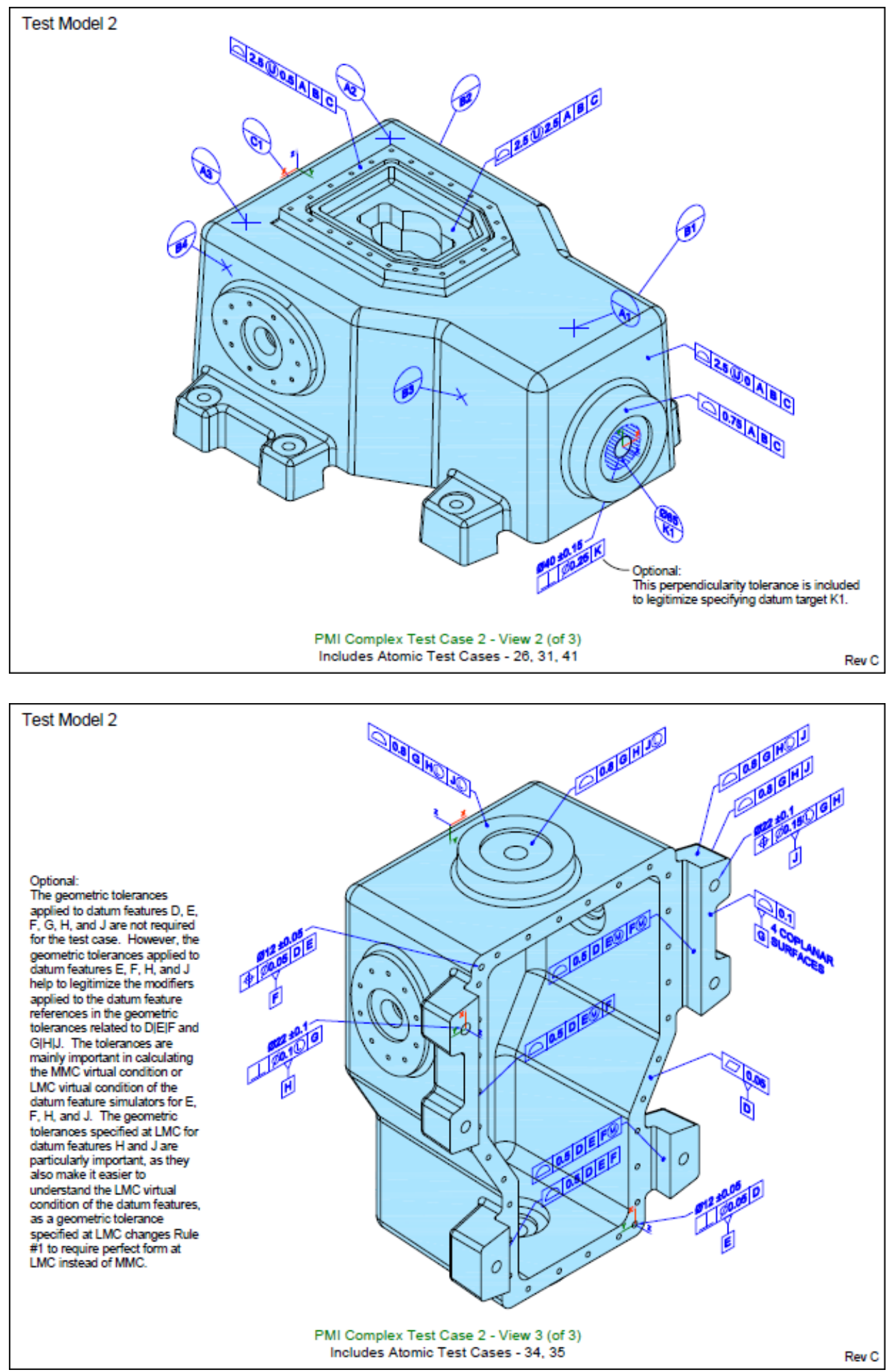

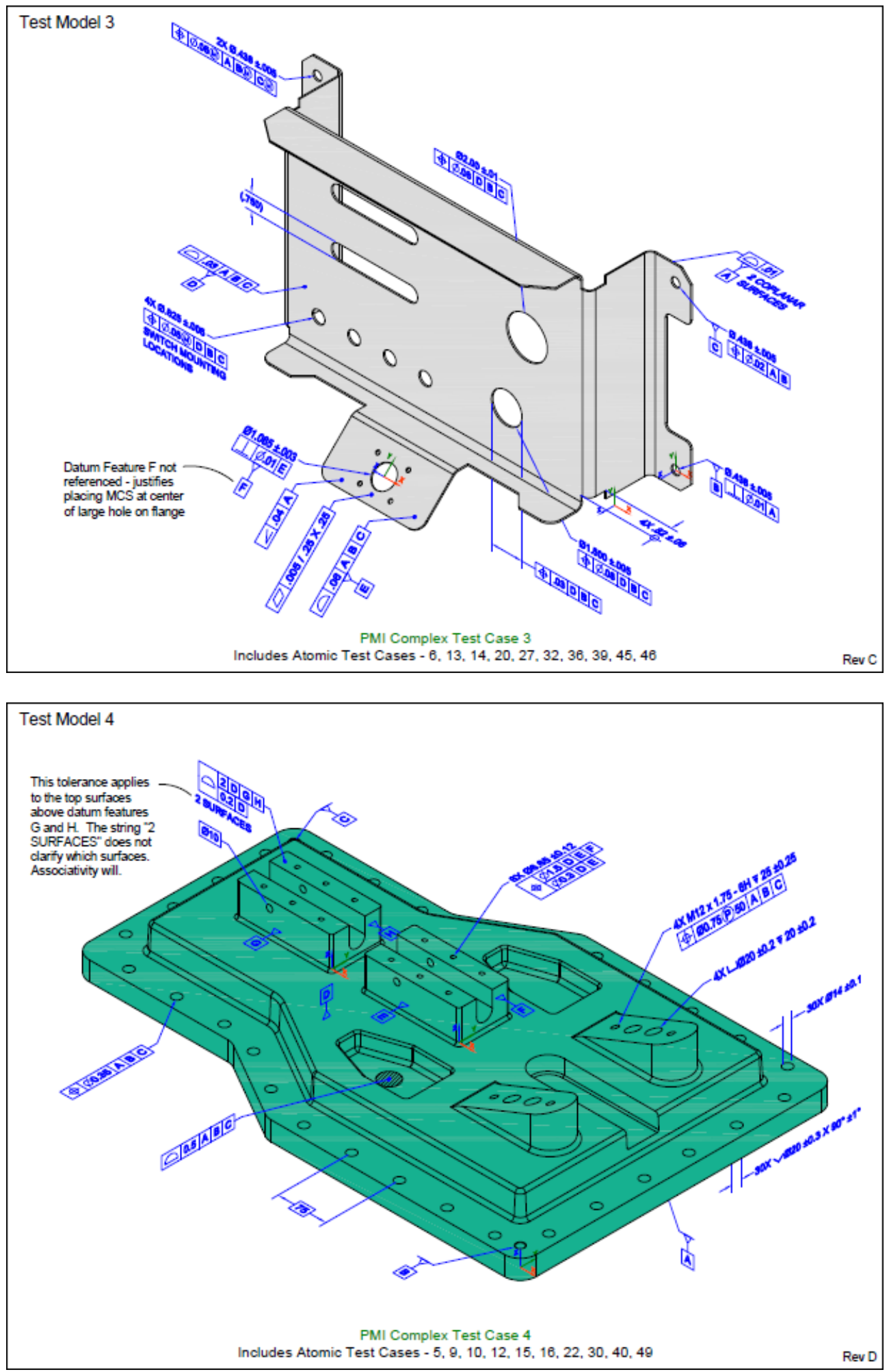

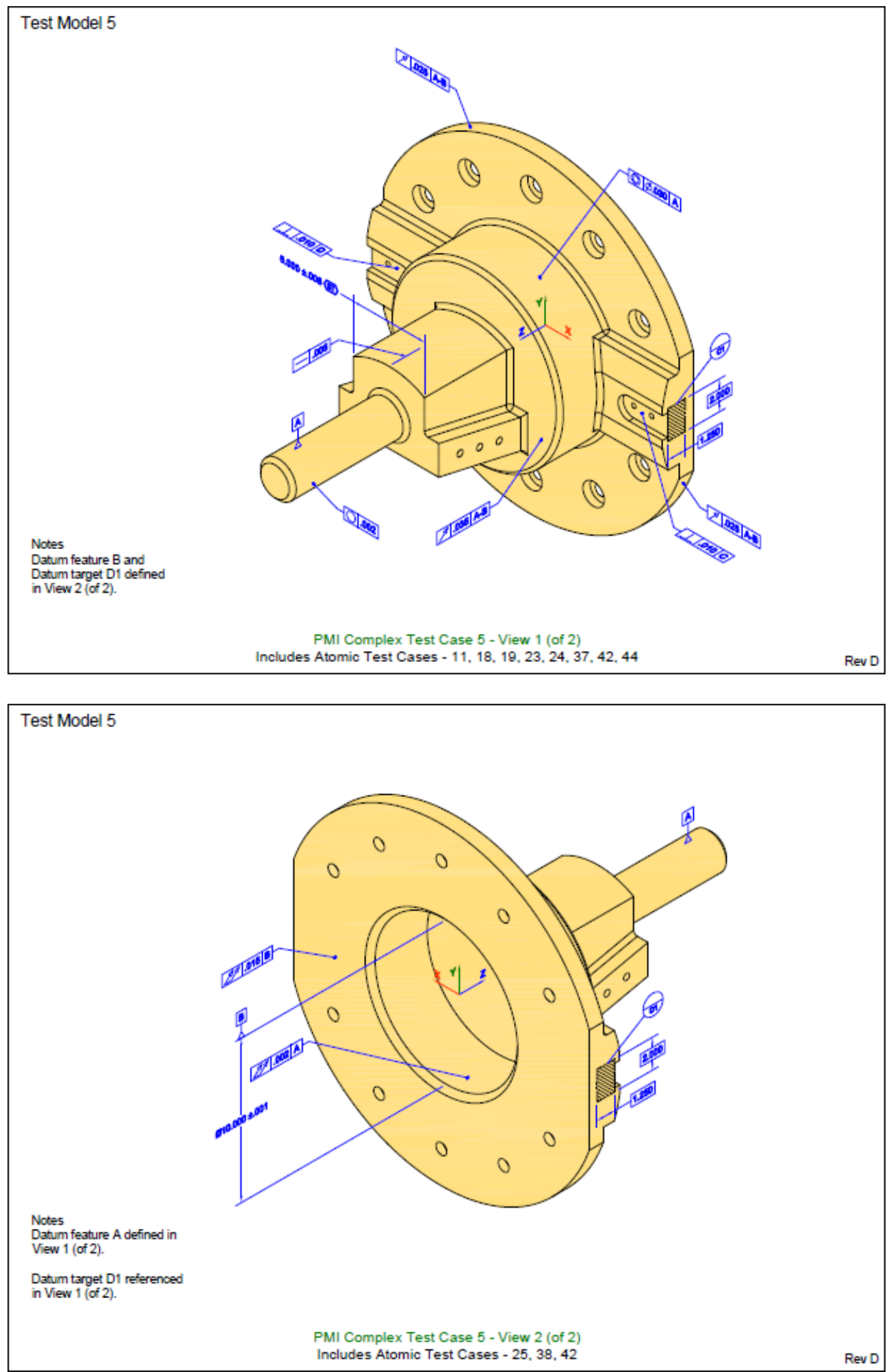


\section{Appendix C: Test Model Images}

\section{Combined Test Case 1}

Saved View MBD_0

- Clockwise from upper left - Test Models for CATIA V5 R21, NX 8.0, SOLIDWORKS 2012 and Creo 2.0

- Screenshot is of each test model displayed in CADIQ

- Annotations and their associated geometry are highlighted in red

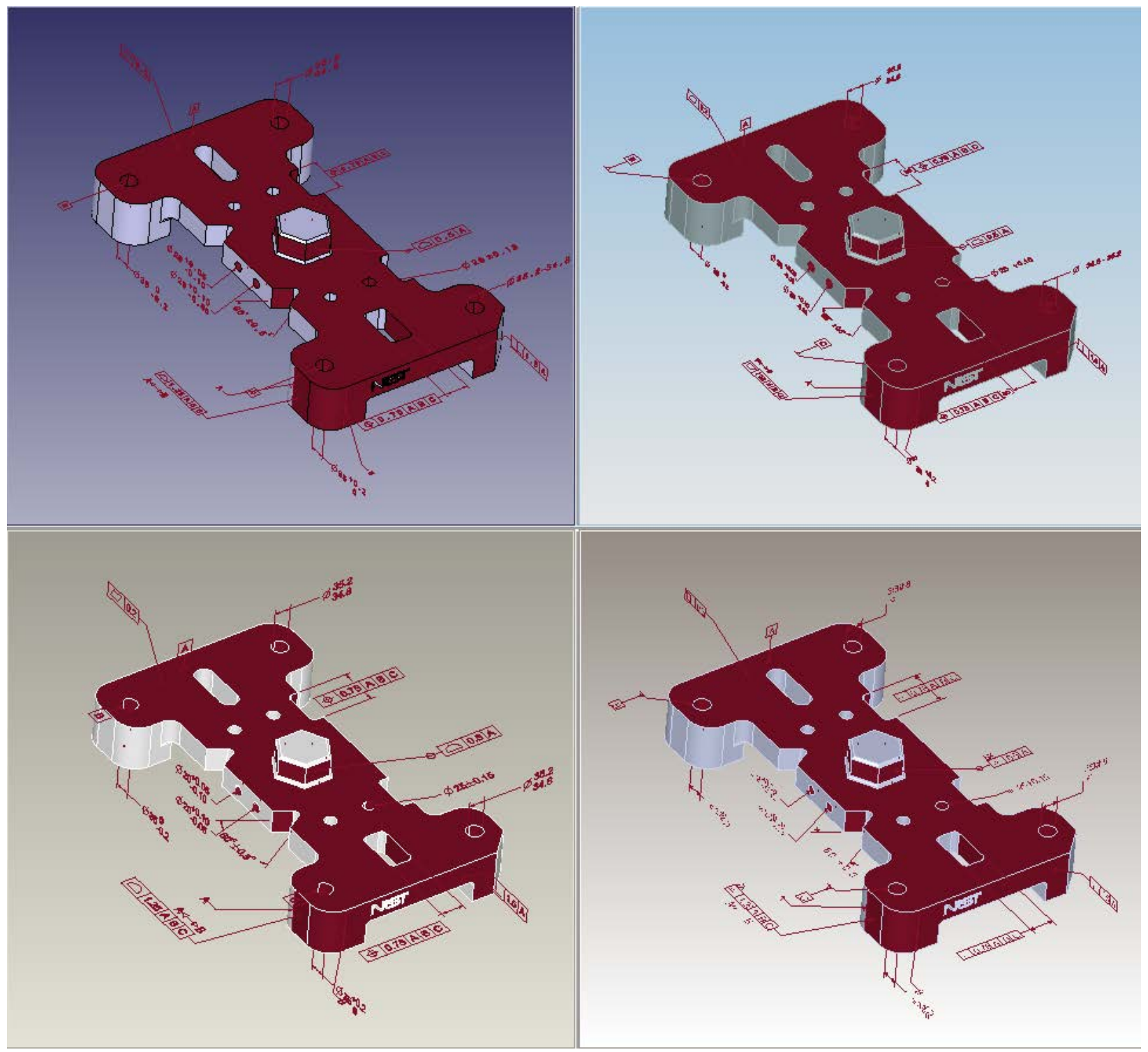




\section{Combined Test Case 2}

Saved View MBD_A

- Clockwise from upper left - Test Models for CATIA V5 R21, NX 8.0, SOLIDWORKS 2012 and Creo 2.0

- Screenshot is of each test model displayed in CADIQ

- Annotations and their associated geometry are highlighted in red

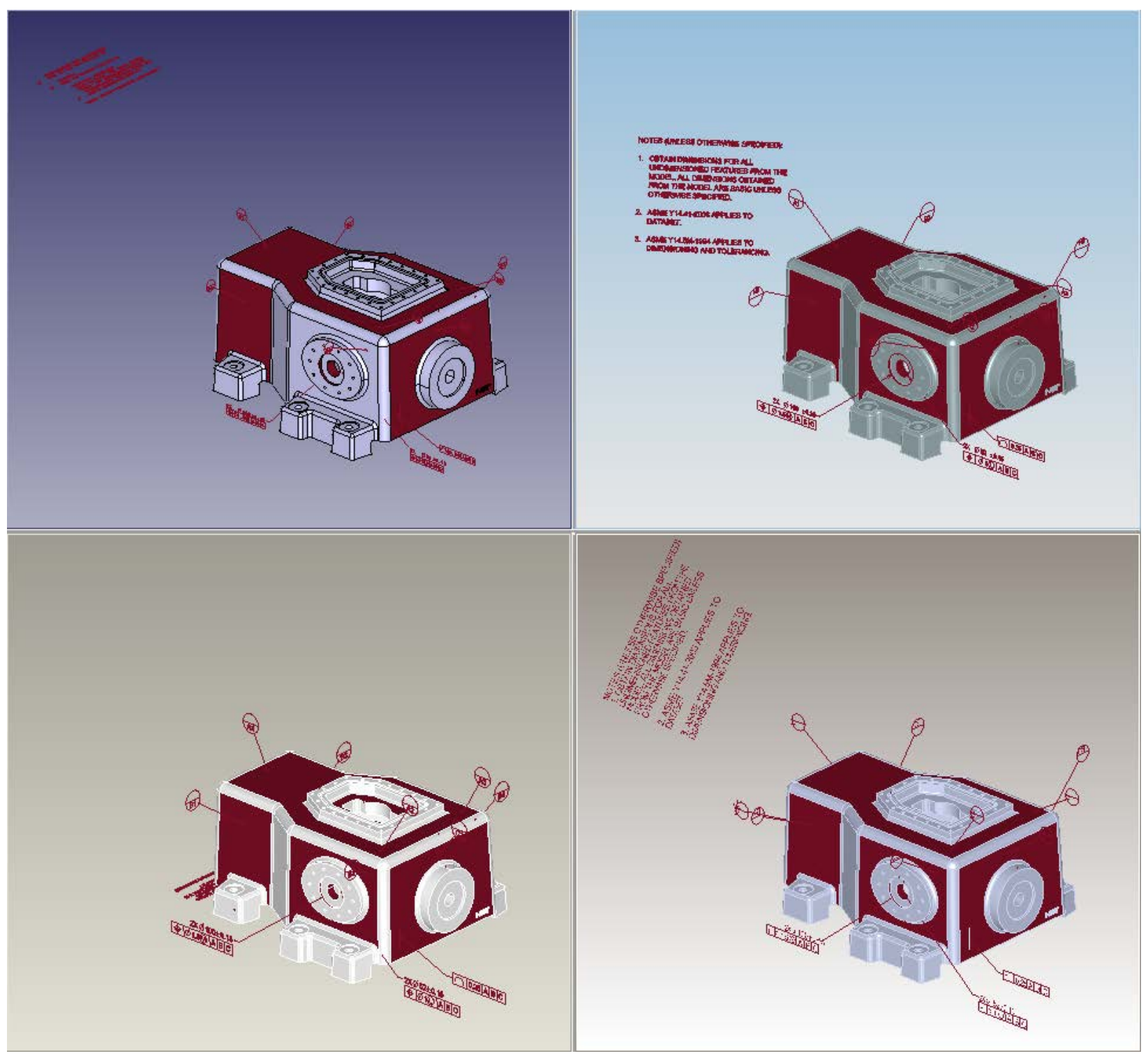




\section{Combined Test Case 2}

Saved View MBD_B

- Clockwise from upper left - Test Models for CATIA V5 R21, NX 8.0, SOLIDWORKS 2012 and Creo 2.0

- Screenshot is of each test model displayed in CADIQ

- Annotations and their associated geometry are highlighted in red

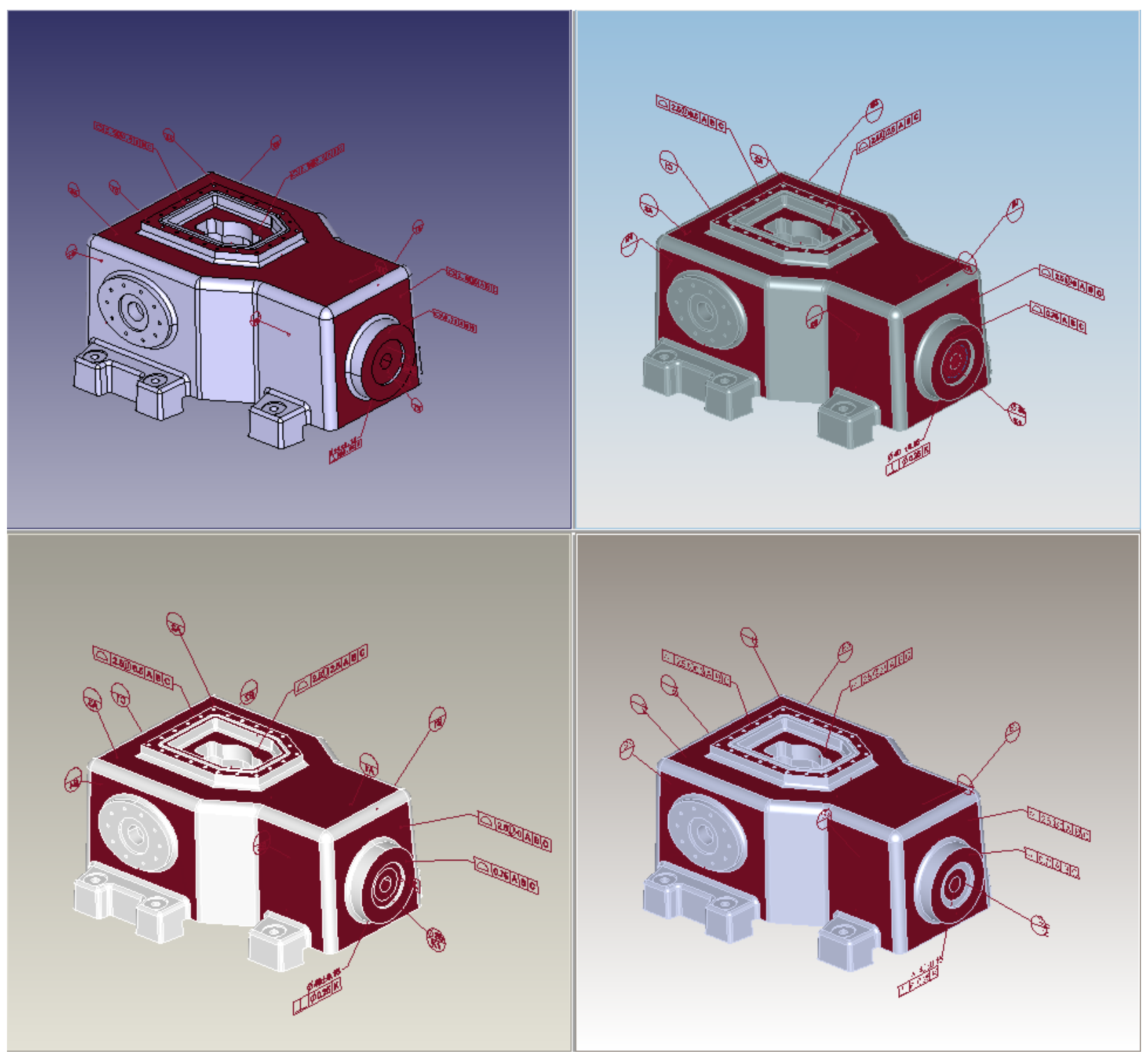




\section{Combined Test Case 2}

Saved View MBD_C

- Clockwise from upper left - Test Models for CATIA V5 R21, NX 8.0, SOLIDWORKS 2012 and Creo 2.0

- Screenshot is of each test model displayed in CADIQ

- Annotations and their associated geometry are highlighted in red

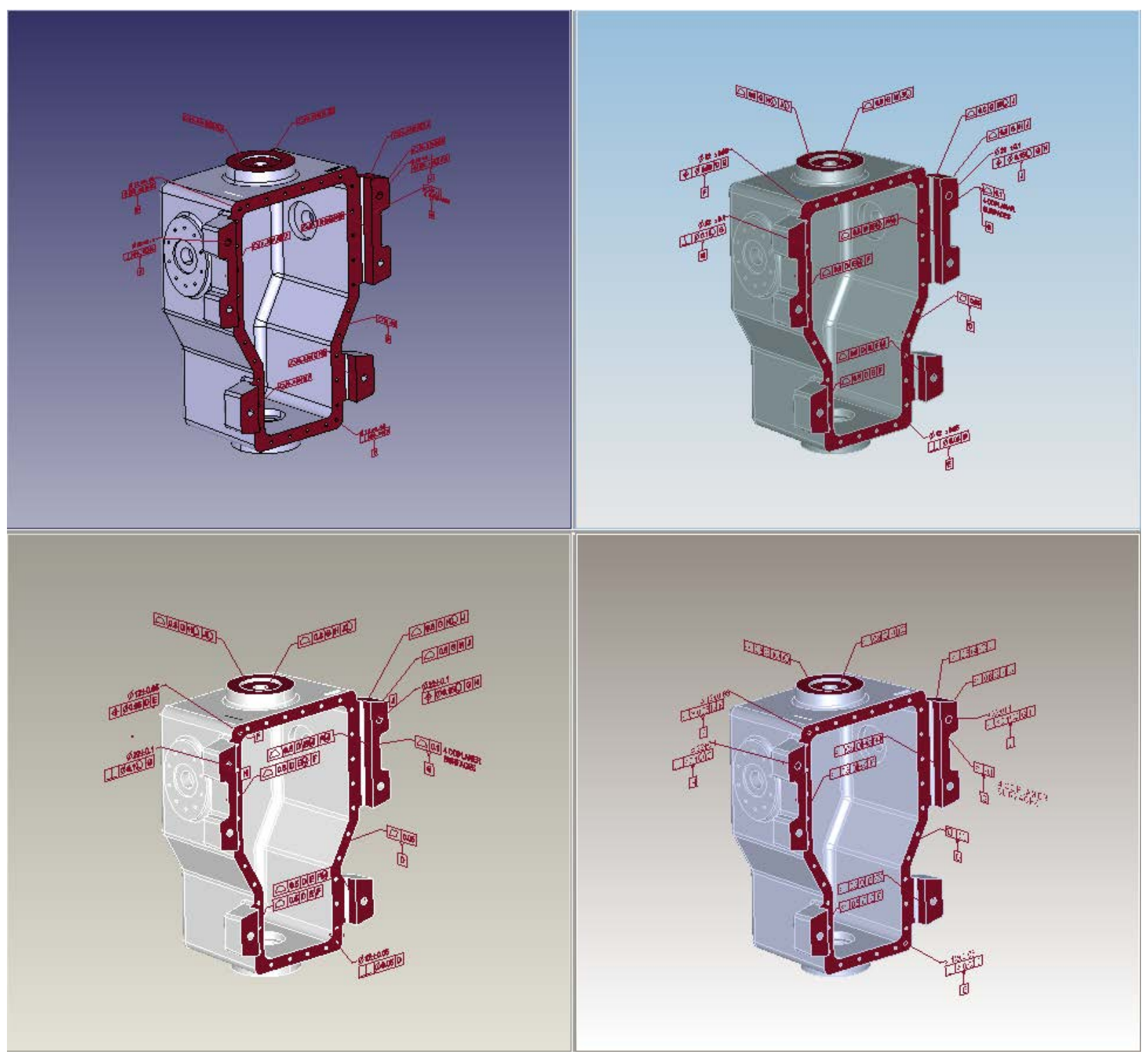




\section{Combined Test Case 3}

Saved View MBD_0

- $\quad$ Clockwise from upper left - Test Models for CATIA V5 R21, NX 8.0, SOLIDWORKS 2012 and Creo 2.0

- Screenshot is of each test model displayed in CADIQ

- Annotations and their associated geometry are highlighted in red

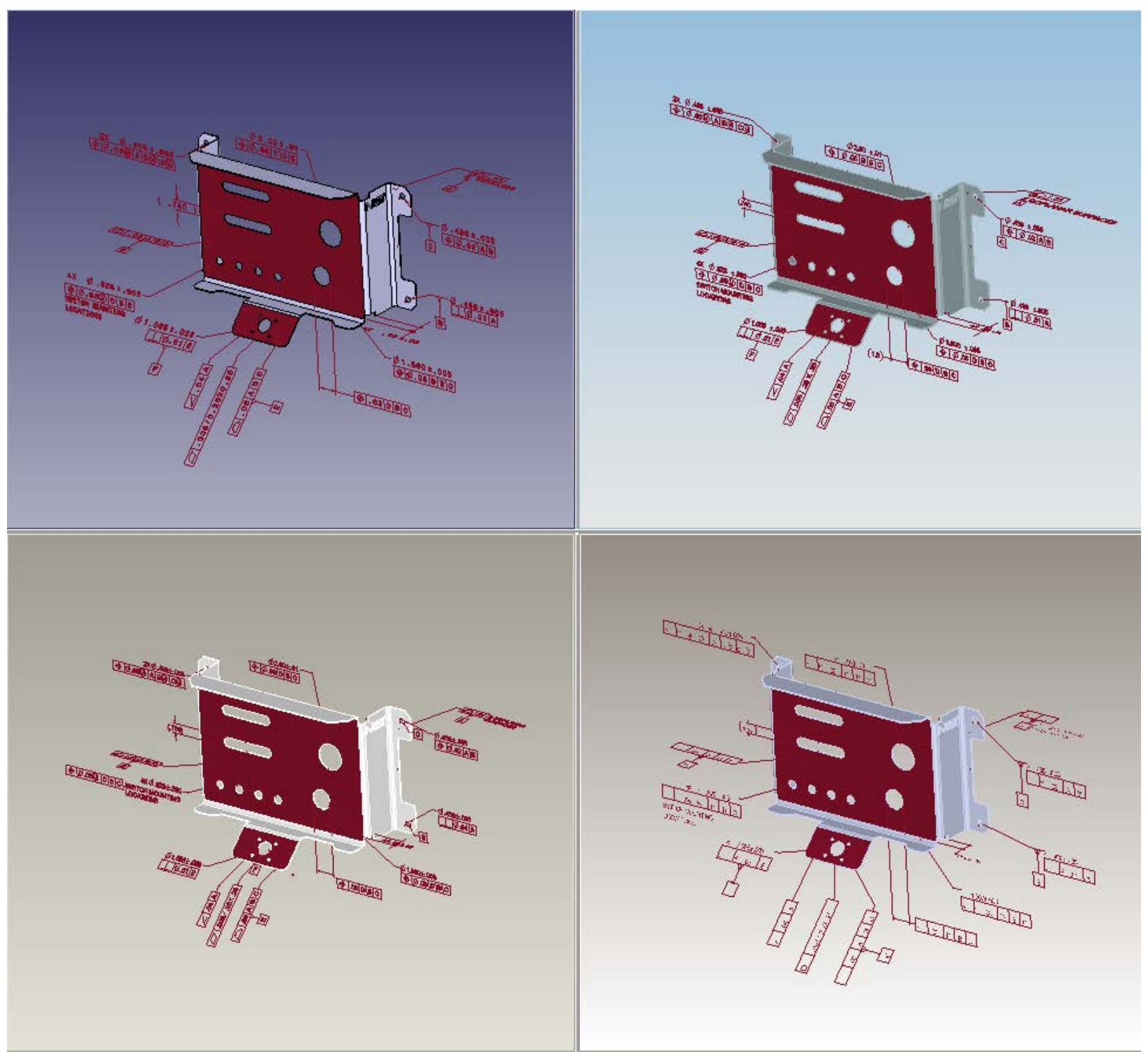




\section{Combined Test Case 4}

Saved View MBD_0

- $\quad$ Clockwise from upper left - Test Models for CATIA V5 R21, NX 8.0, SOLIDWORKS 2012 and Creo 2.0

- Screenshot is of each test model displayed in CADIQ

- Annotations and their associated geometry are highlighted in red

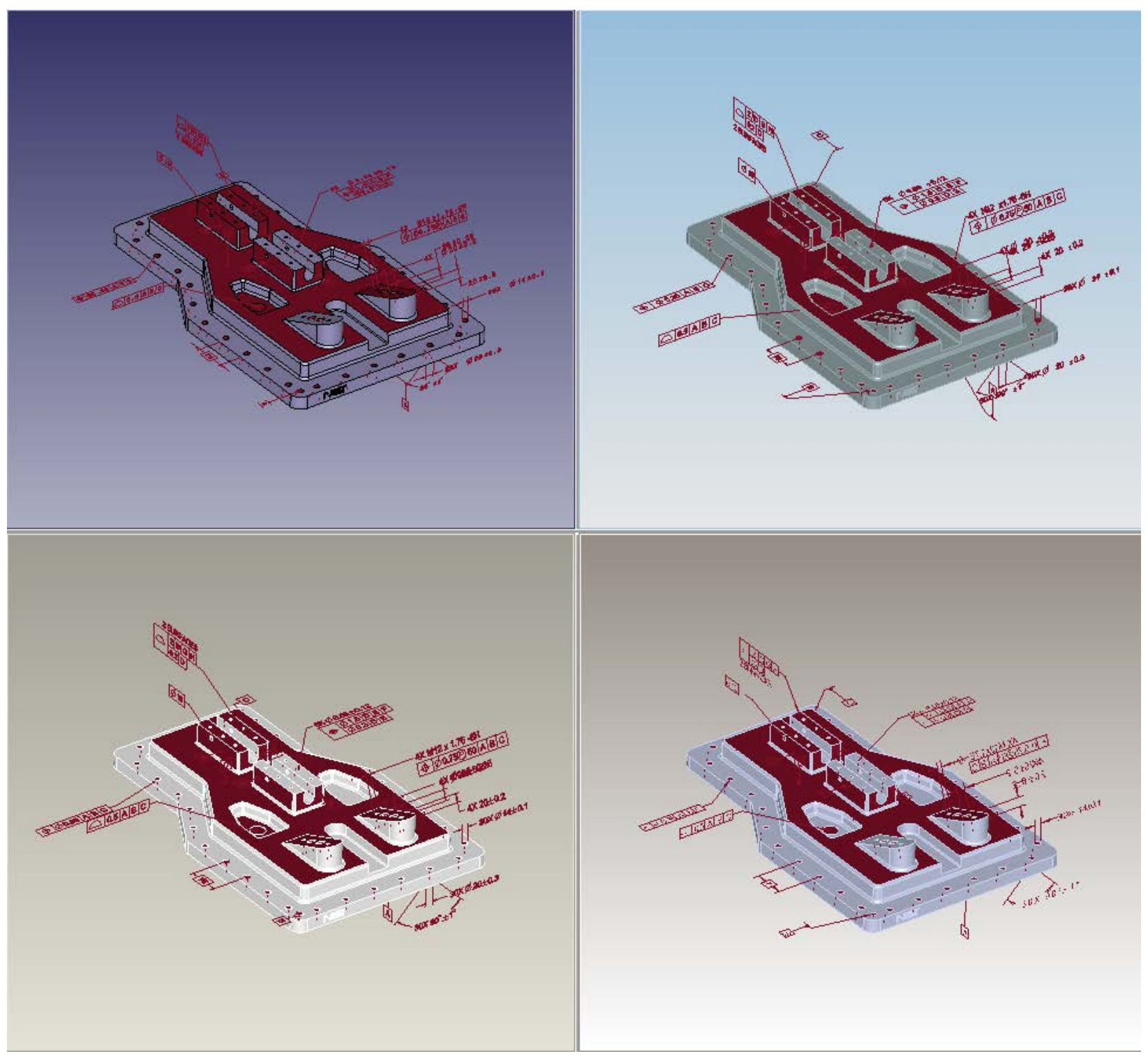




\section{Combined Test Case 5}

Saved View MBD_A

- Clockwise from upper left - Test Models for CATIA V5 R21, NX 8.0, SOLIDWORKS 2012 and Creo 2.0

- Screenshot is of each test model displayed in CADIQ

- Annotations and their associated geometry are highlighted in red

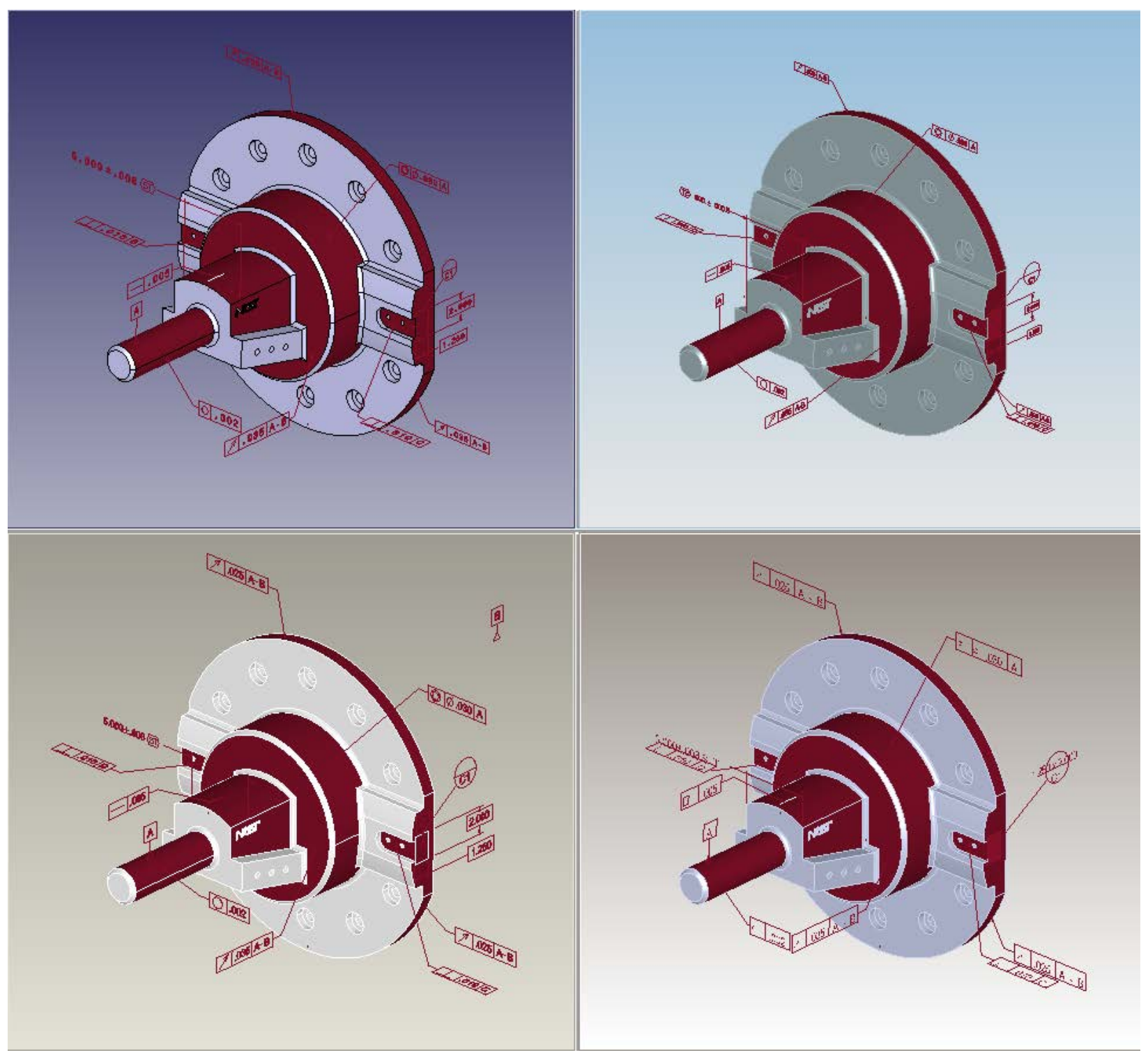




\section{Combined Test Case 5}

Saved View MBD_B

- Clockwise from upper left - Test Models for CATIA V5 R21, NX 8.0, SOLIDWORKS 2012 and Creo 2.0

- Screenshot is of each test model displayed in CADIQ

- Annotations and their associated geometry are highlighted in red

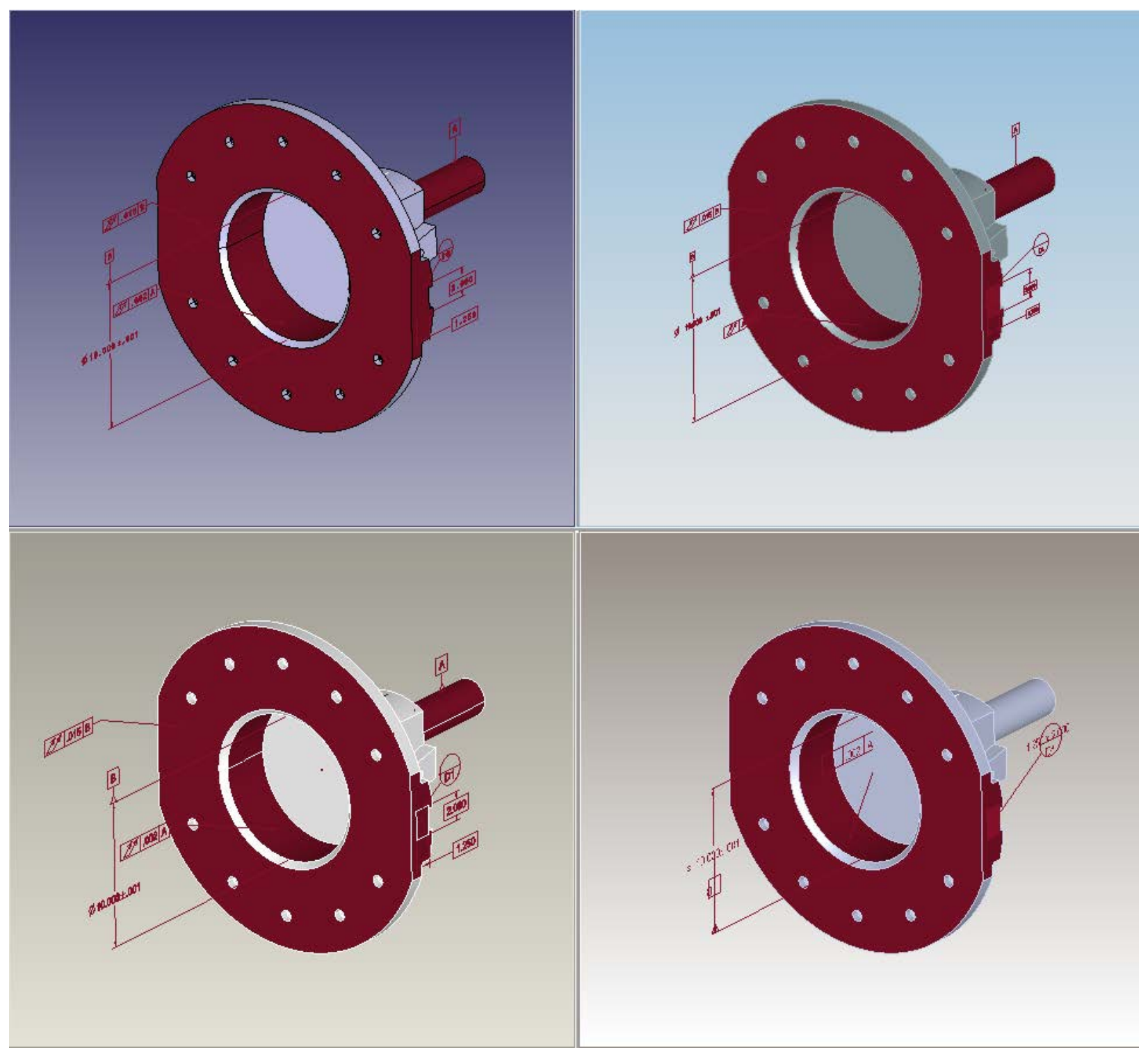


Appendix D: Representation Limitation Examples

Annotation Structure:

Representation Limitation Countersink diameter DIM not defined

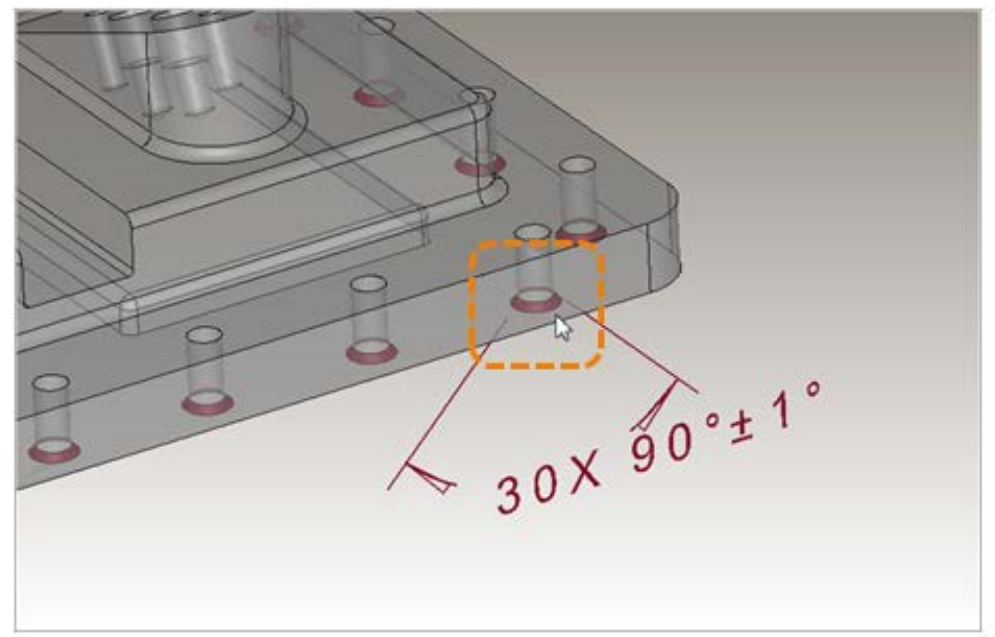

The diameter dimension for the outside (larger, circular) edge of this countersink is not defined.

Annotation Structure:

Representation Limitation DIM defined as part of DTS

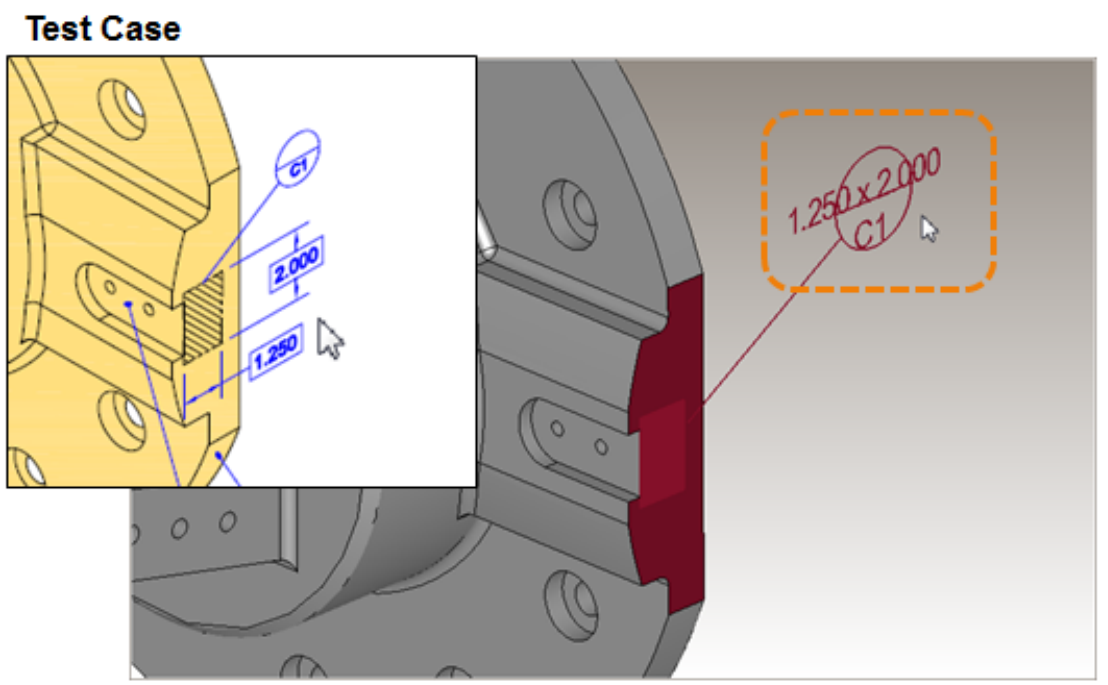

The dimensions for the target area of this datum target are represented as parameters in the datum target symbol and not as separate dimensions. 
Annotation Structure:

Representation Limitation

FCF extension line defined as separate DIM

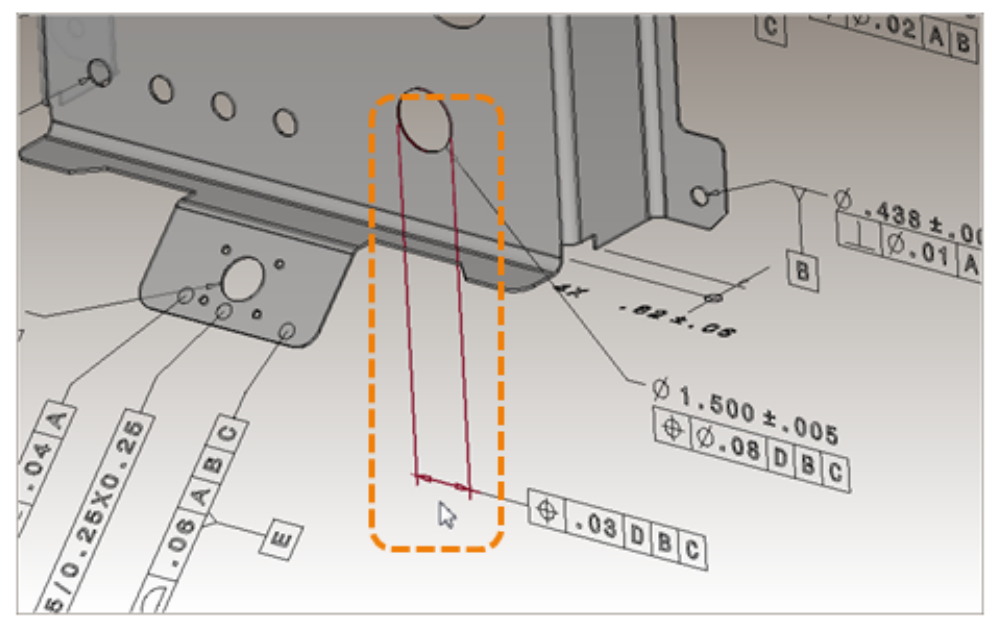

The extension lines for this feature control frame, whose orientation is critical to the tolerance zone definition, are defined as a separate dimension with no displayed value.

Annotation Structure:

Representation Limitation

\section{FCF projected tolerance zone defined as separate DIM}

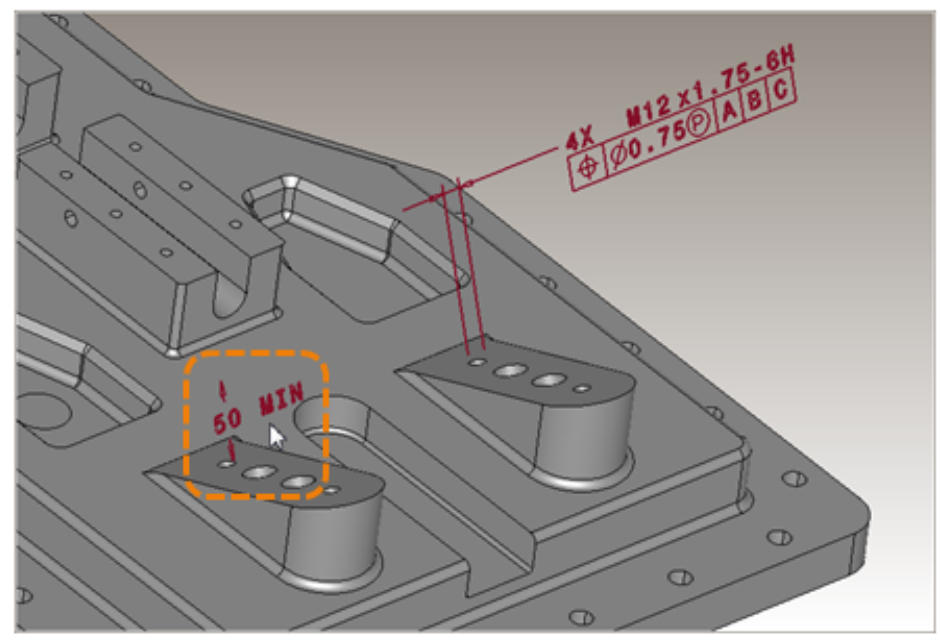

The length of the projected tolerance zone for this feature control frame is defined as a separate dimension.

(See related Presentation Limitation) 
Annotation Structure:

Representation Limitation

FCF text defined as separate note

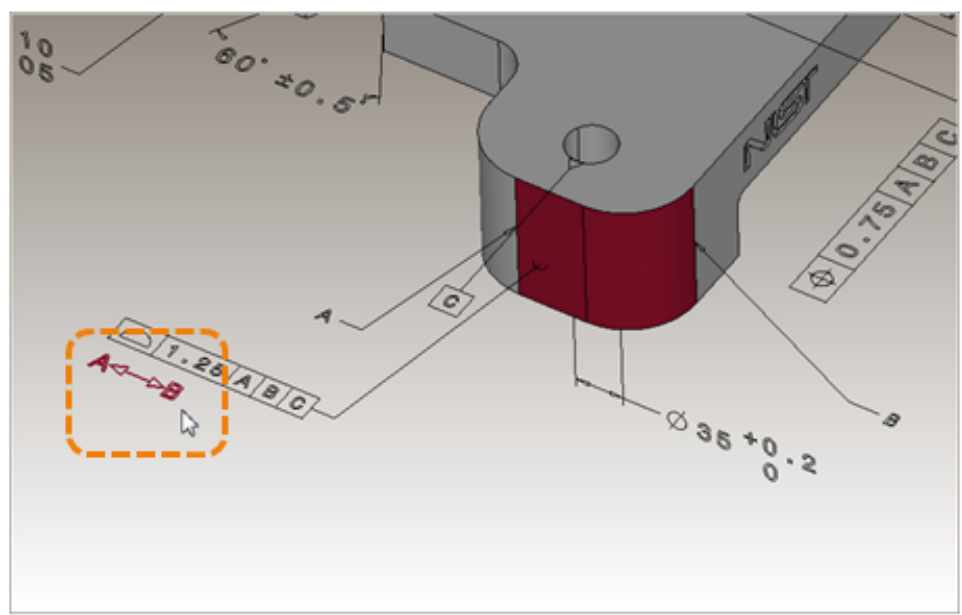

The text which defines the between-basis for this feature control frame is defined as a separate note annotation.

\section{Annotation Structure:}

Representation Limitation

Threaded hole depth DIM not defined

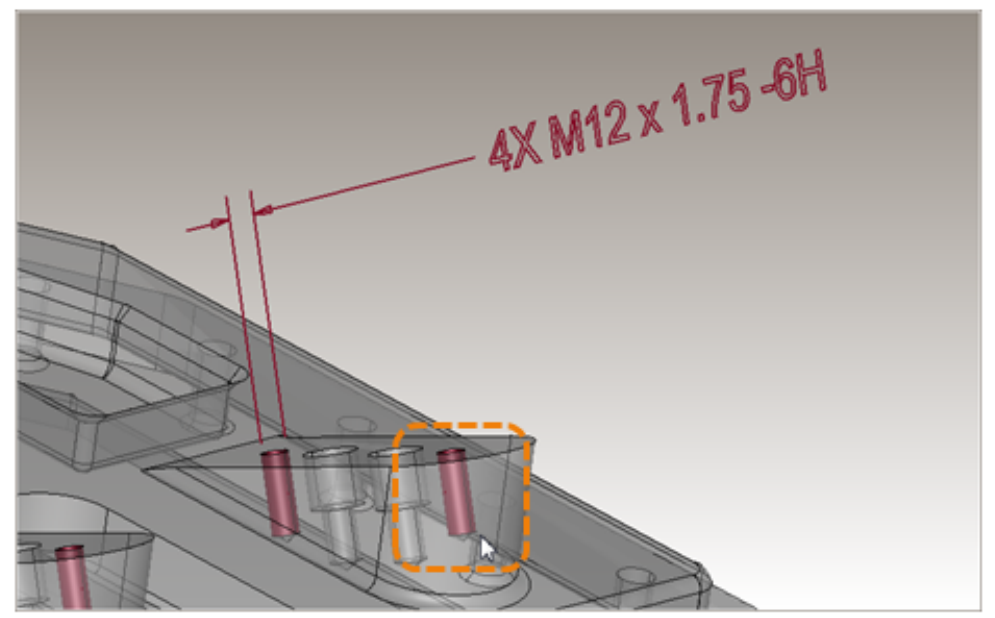

The depth dimension for each threaded hole, between the top (flat) surface and the bottom (circular) edge, is not defined. 
Annotation Parameters:

Representation Limitation DIM origin not defined

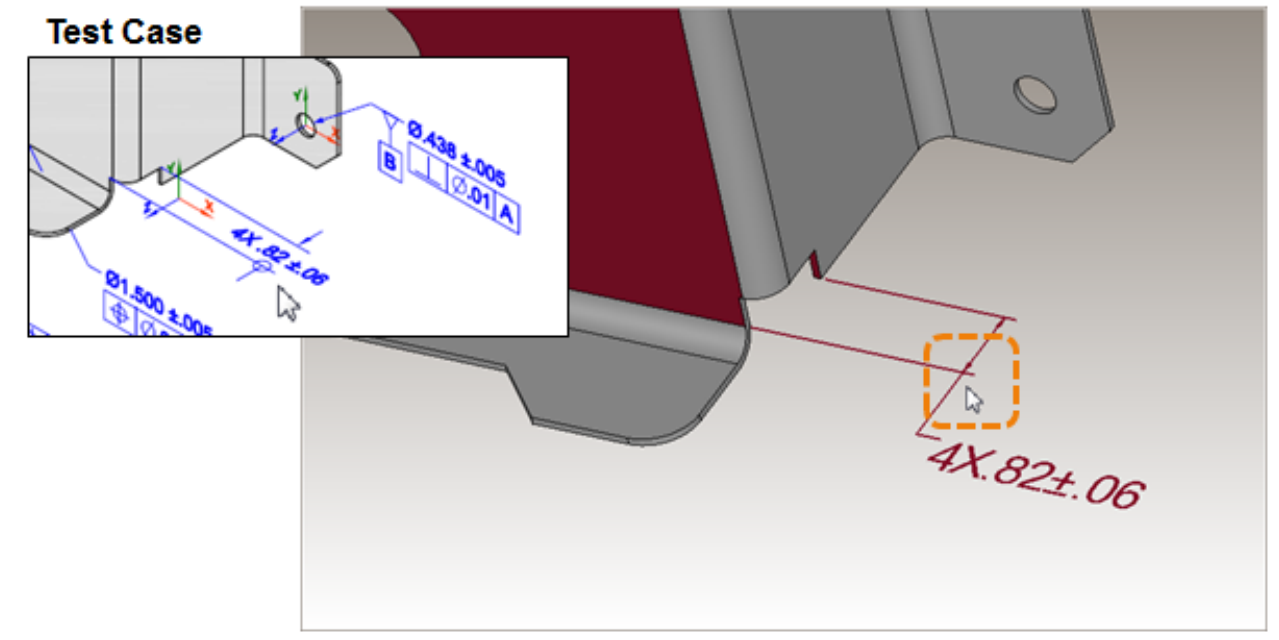

The origin for this oriented dimension is not defined.

Annotation Parameters:

Representation Limitation

DIM parameter defined with encoded text

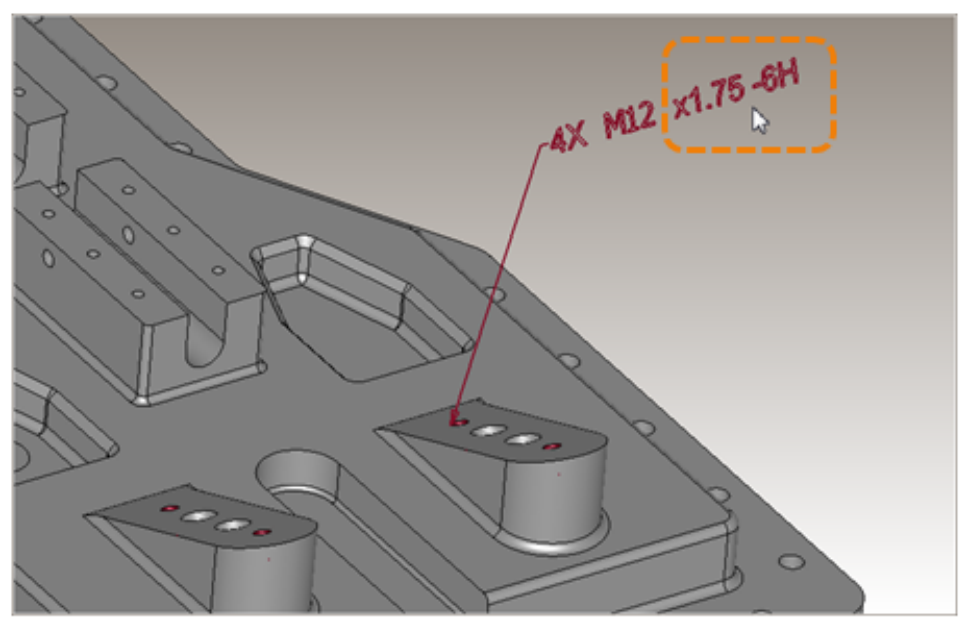

The thread parameters for this threaded hole dimension are defined as encoded text and not as named parameters. 
Annotation Parameters:

Representation Limitation

FCF between-basis defined with encoded text

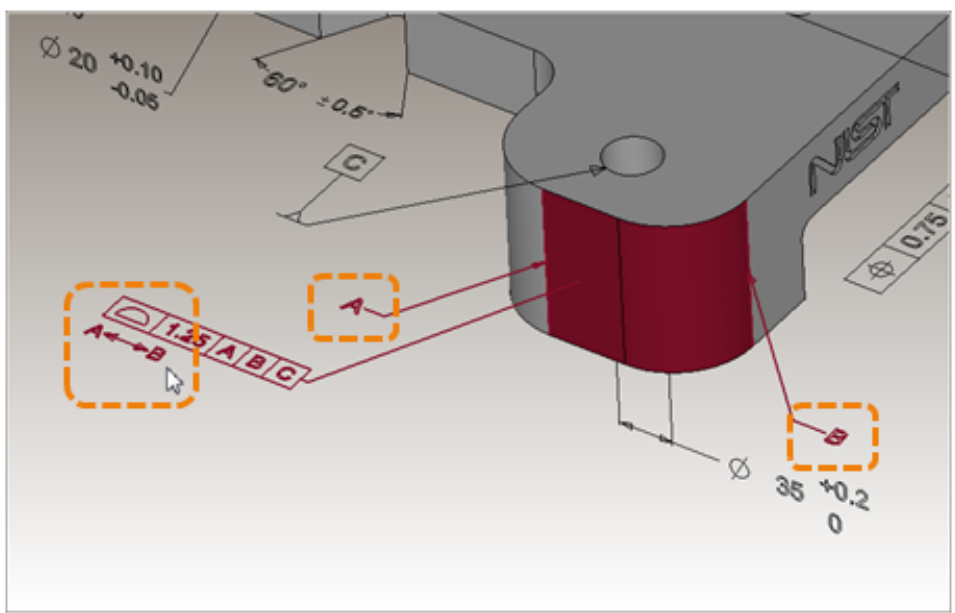

The between-basis for this feature control frame is defined as encoded text and not with named parameters.

Annotation Parameters:

Representation Limitation

FCF parameter defined with encoded text

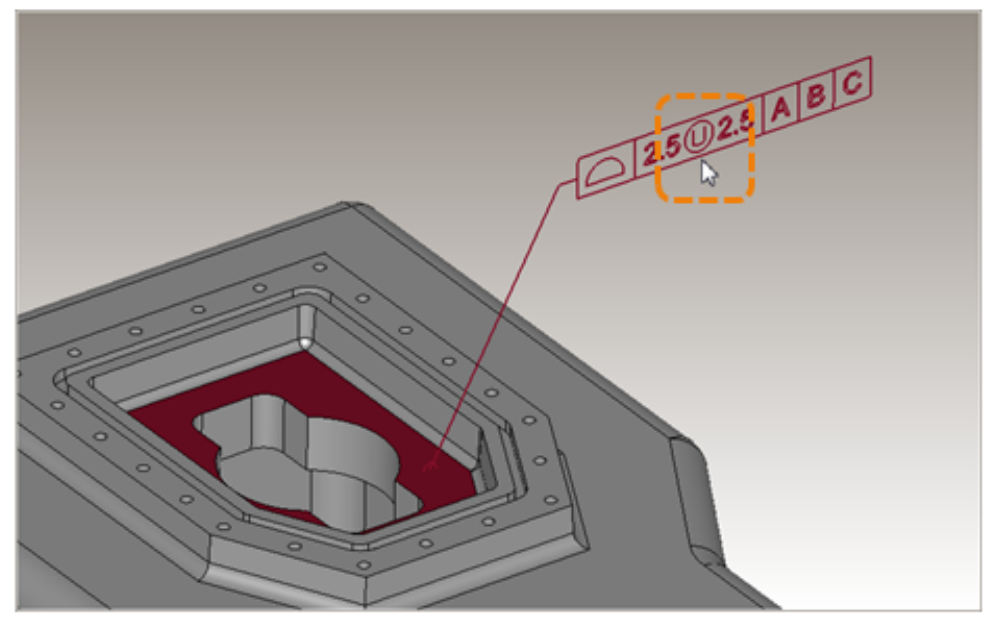

The unequally disposed modifier in this feature control frame is defined as a text symbol and not as a named parameter. 


\section{Annotation Geometry: \\ Representation Limitation \\ DIM associated with extra face}

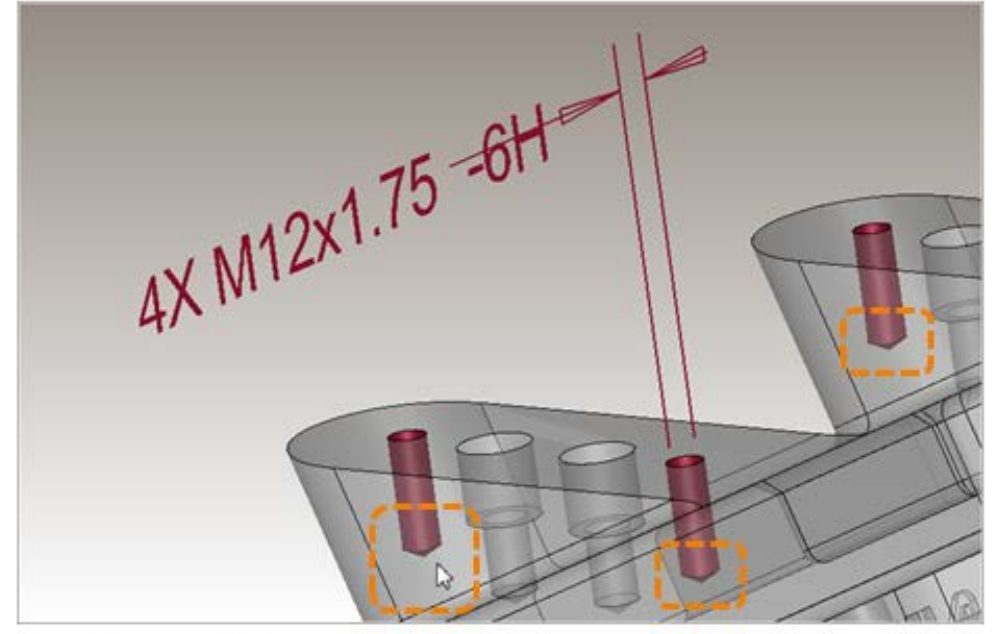

This hole diameter dimension is associated with the bottom face of each hole and not just the side faces.

\section{Annotation Geometry: \\ Representation Limitation DIM not associated with complete set of faces}

Complete Representation

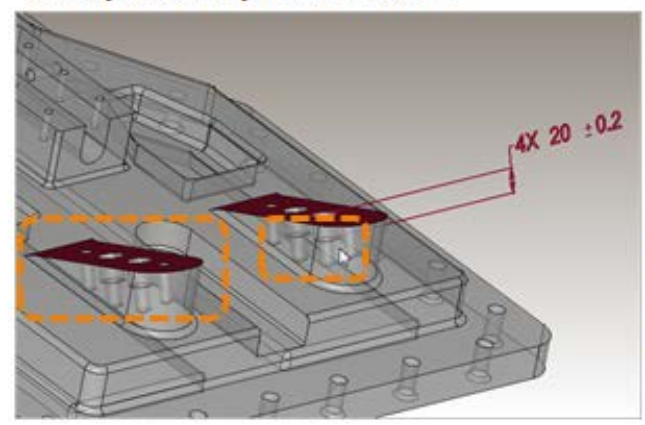

Incomplete Representation

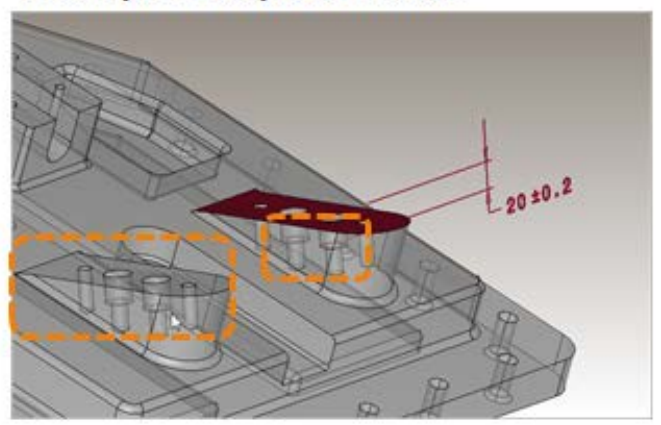

This counterbore depth dimension is not associated with both planar faces and all 4 bottom faces. 
Annotation Geometry:

Representation Limitation

DTS associated with extra face

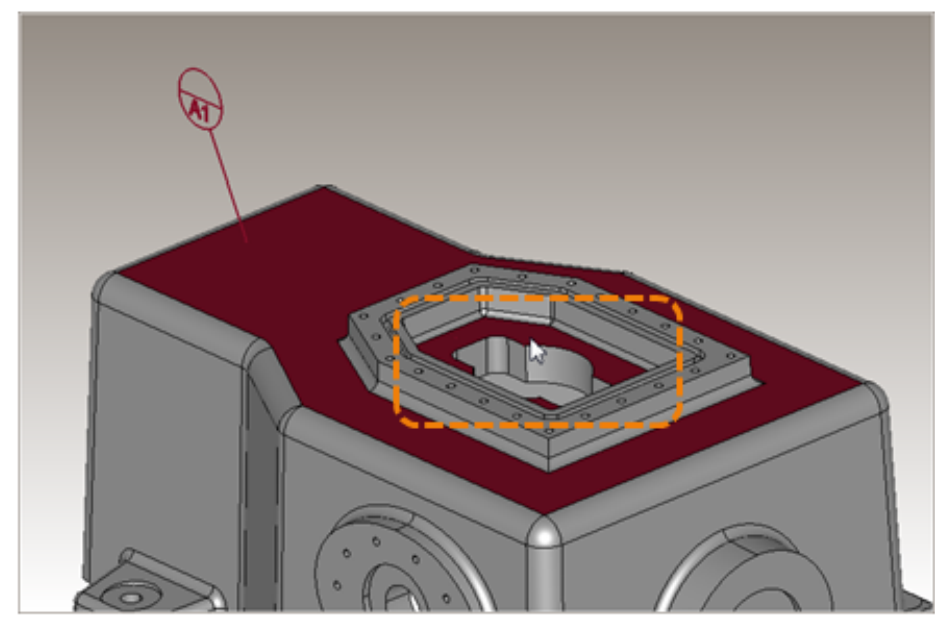

This datum target symbol is associated with an extra (coplanar) face and not just the specified face.

Annotation Geometry:

Representation Limitation

DTS not associated with face

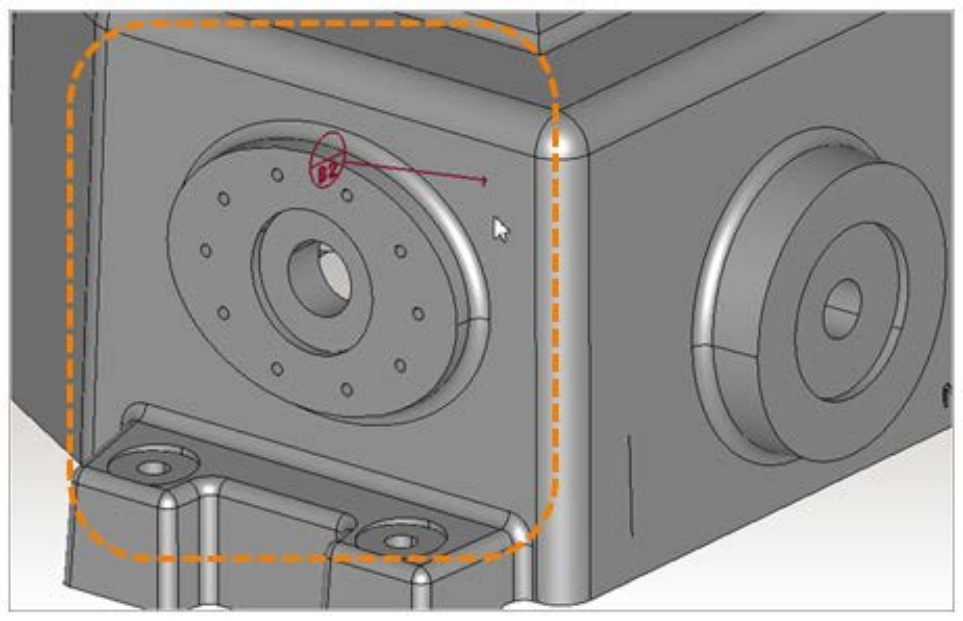

This datum target symbol is not associated with the face on which the datum target is located. 
Annotation Geometry:

Representation Limitation

DTS not associated with SG point

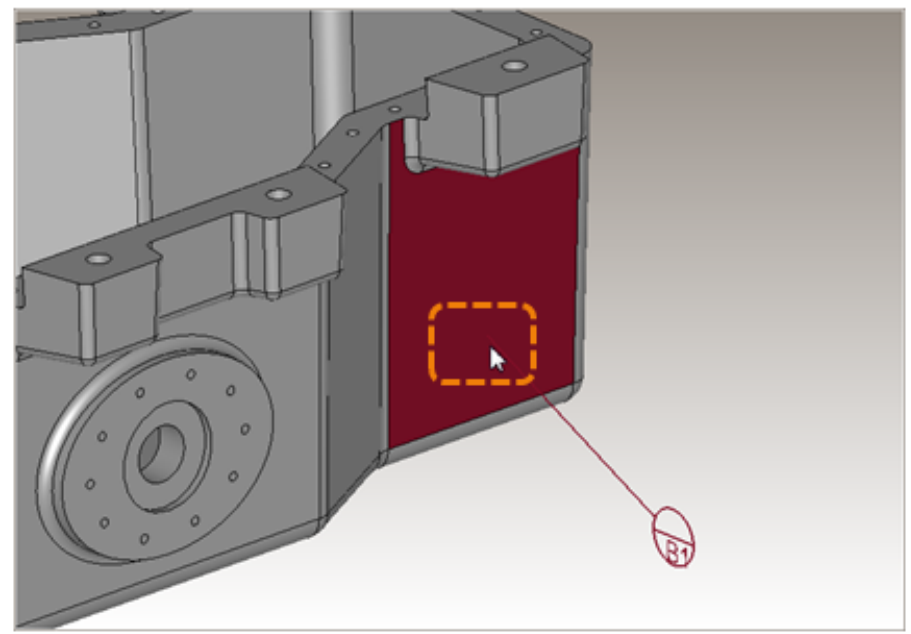

This datum target symbol is not associated with the supplemental geometry point that defines its location on the face.

Annotation Geometry:

Representation Limitation

FCF associated with extra face

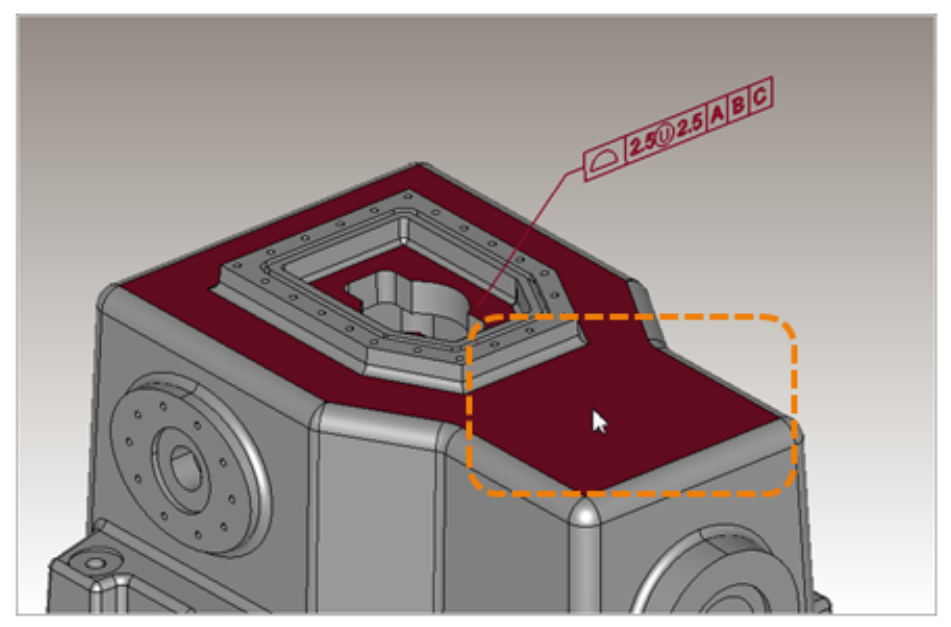

This feature control frame is associated with an extra (coplanar) face and not just the specified face. 
Annotation Geometry:

Representation Limitation

\section{FCF not associated with SG curve}

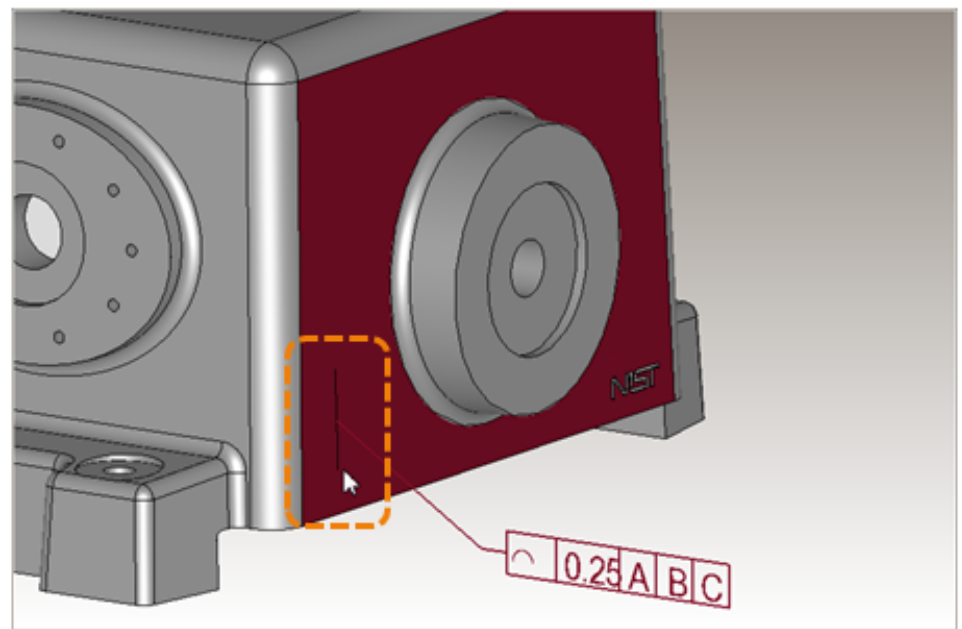

This feature control frame is not associated with the supplemental geometry curve that defines its profile direction on this face.

Coordinate System Structure:

Representation Limitation CS not linked to FCF DRF

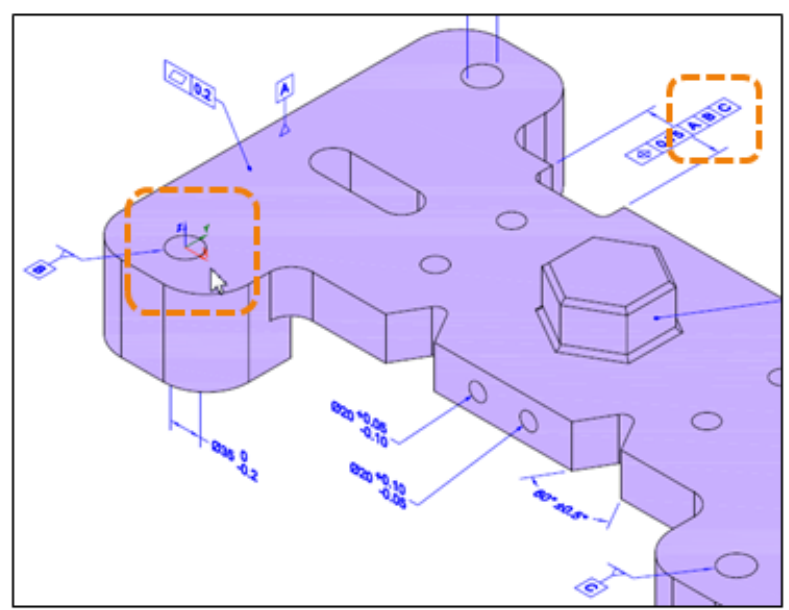

The model has no explicit (named) link from each feature control frame to the coordinate system that represents its datum reference frame. 
Appendix E: Presentation Limitation Examples

Annotation Visibility: DFS is extraneous when DTS is defined

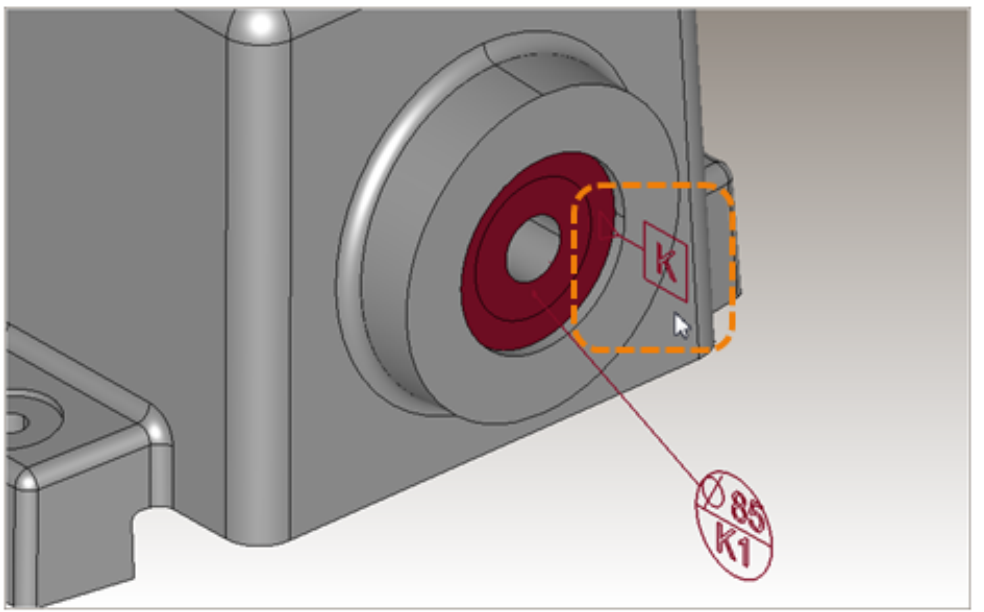

This datum feature symbol is not needed when the datum target symbol is defined. But the system will not allow it to be removed or hidden from this saved view.

\section{Annotation Visibility:}

\section{DFS not visible in specified view}

\section{Test Case}
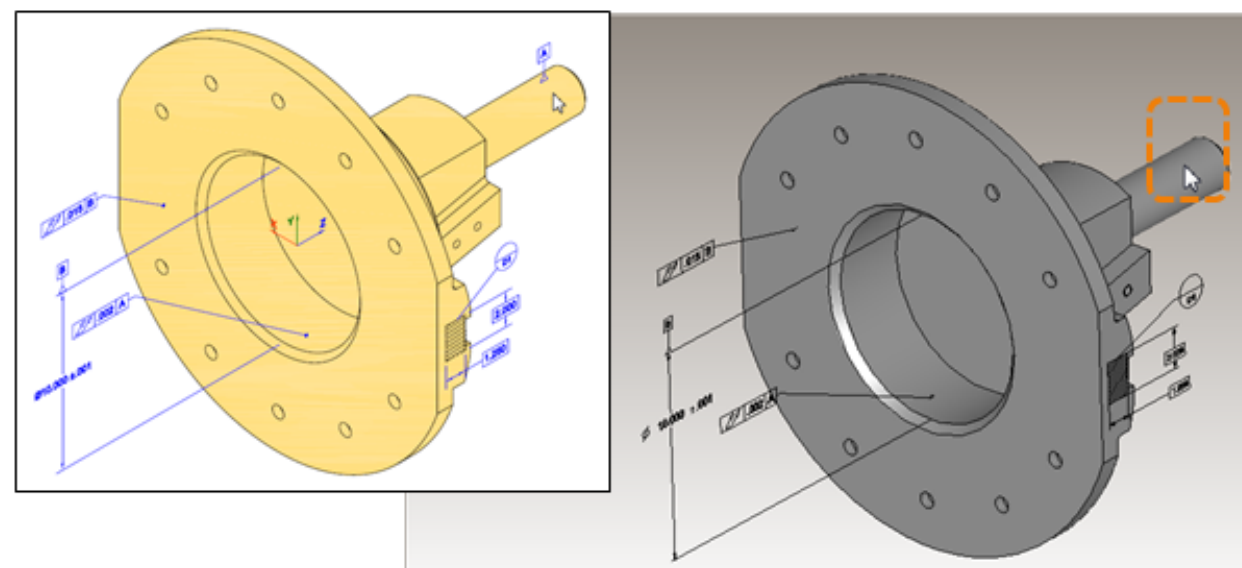

This model has datum feature symbol " $\mathrm{A}$ " defined in the first saved view. But it cannot also be displayed in the second saved view as specified. 
Annotation Visibility:

\section{DIM not visible in specified view}

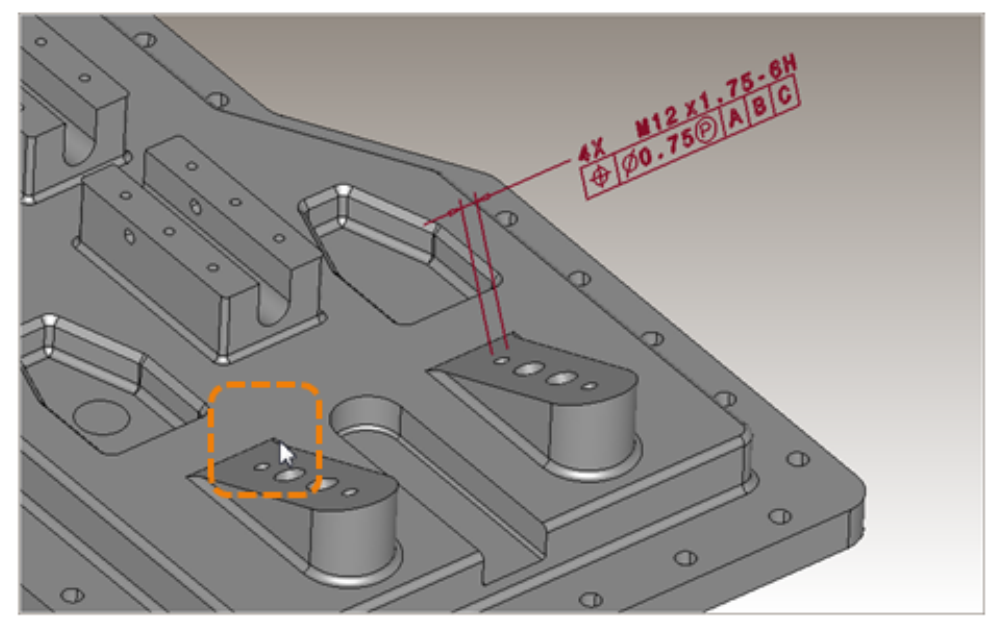

The length of the projected tolerance zone for this feature control frame is defined as a separate dimension. (See related Representation Limitation) This dimension cannot be displayed in this saved view.

Annotation Visibility:

Presentation Limitation DTS visible in wrong view

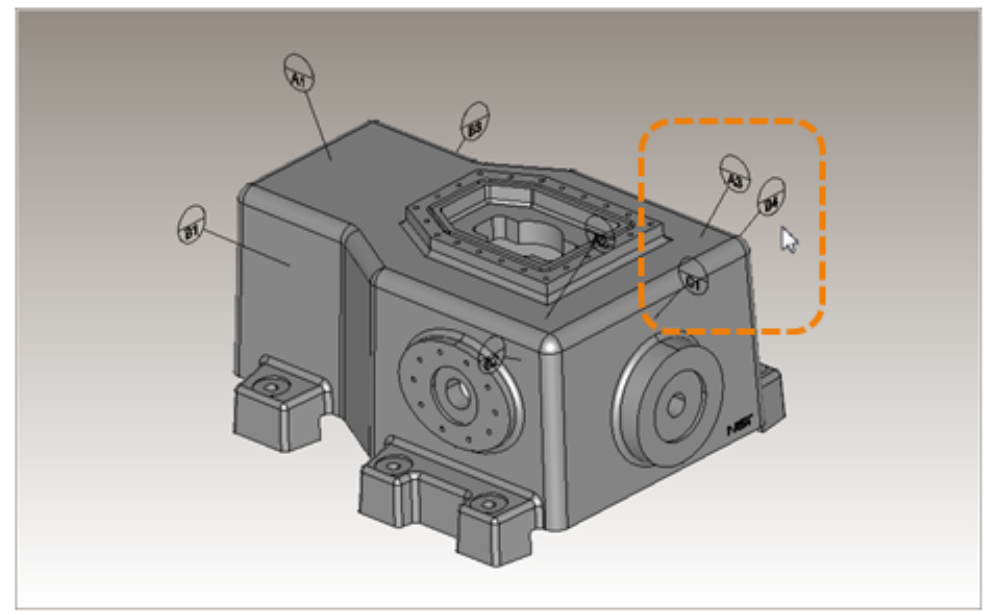

These datum target symbols are visible in a default (unspecified) saved view which cannot be deleted from the model. 

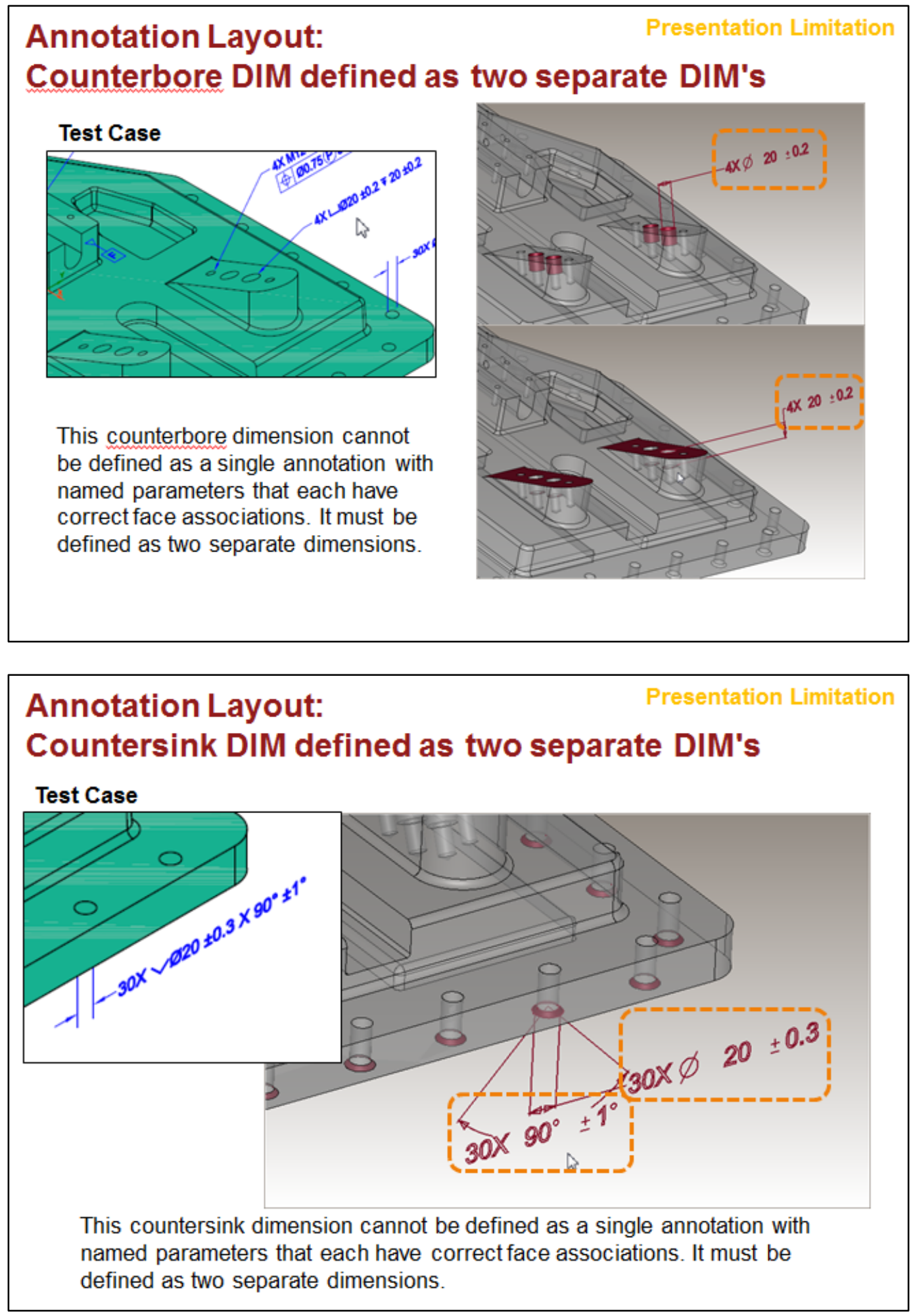
Annotation Layout:

Presentation Limitation

\section{Threaded hole DIM defined as two separate DIM's}

\section{Test Case}

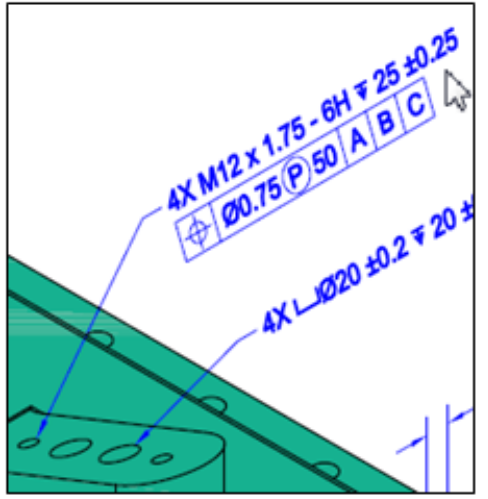

This threaded hole dimension cannot be defined as a single annotation with named parameters that each have correct face associations. It must be defined as two separate dimensions.

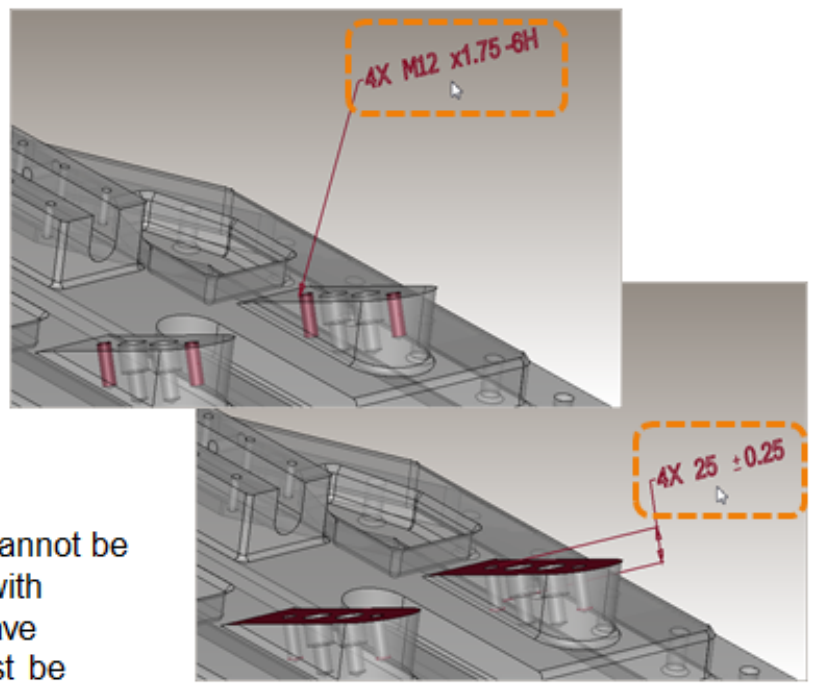

Annotation Layout:

Presentation Limitation DIM limits displayed in reversed order

Test Case

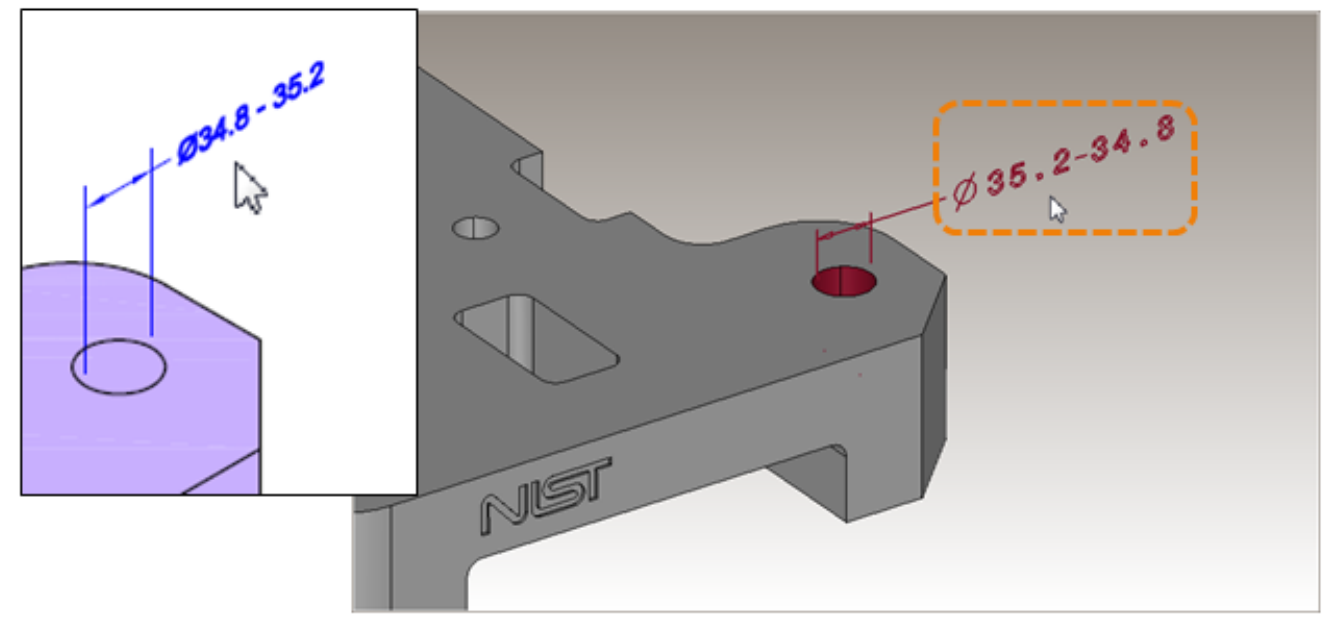

The lower and upper limits of this dimension are displayed in the reverse order from what is specified. 


\section{Annotation Layout: \\ Presentation Limitation \\ DIM limits not displayed horizontally}

\section{Test Case}

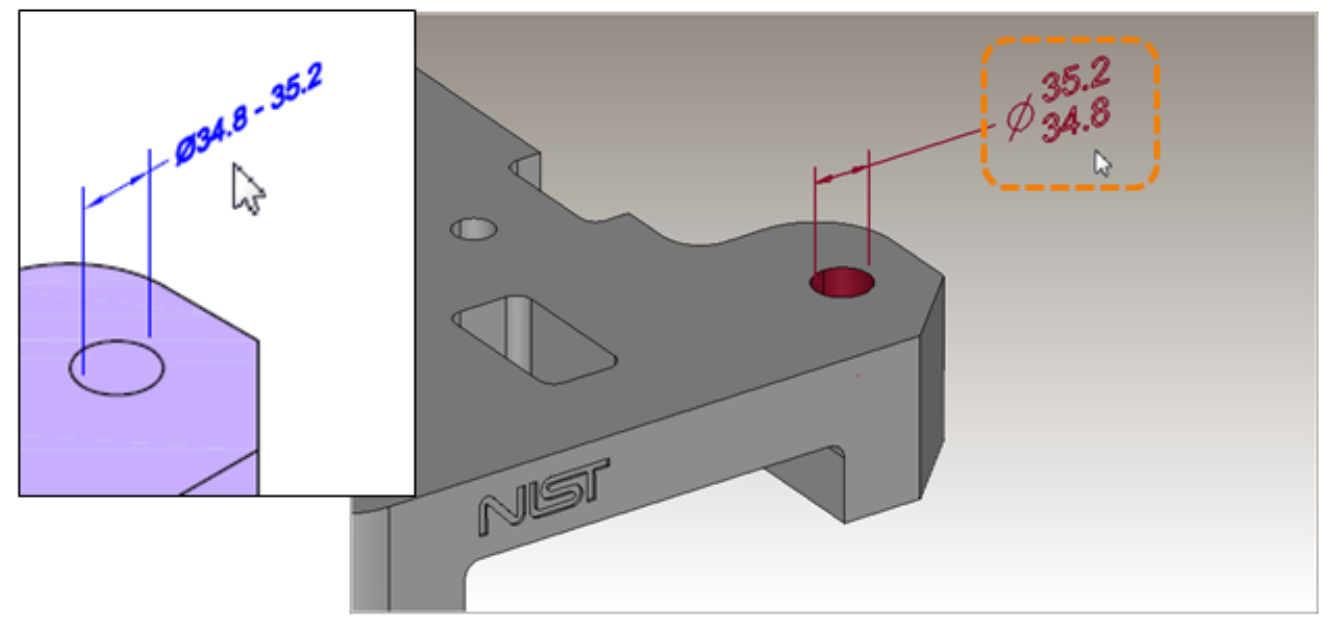

The lower and upper limits of this dimension are not displayed horizontally as specified.

Annotation Layout:

Presentation Limitation DTS target area diameter defined as separate DIM
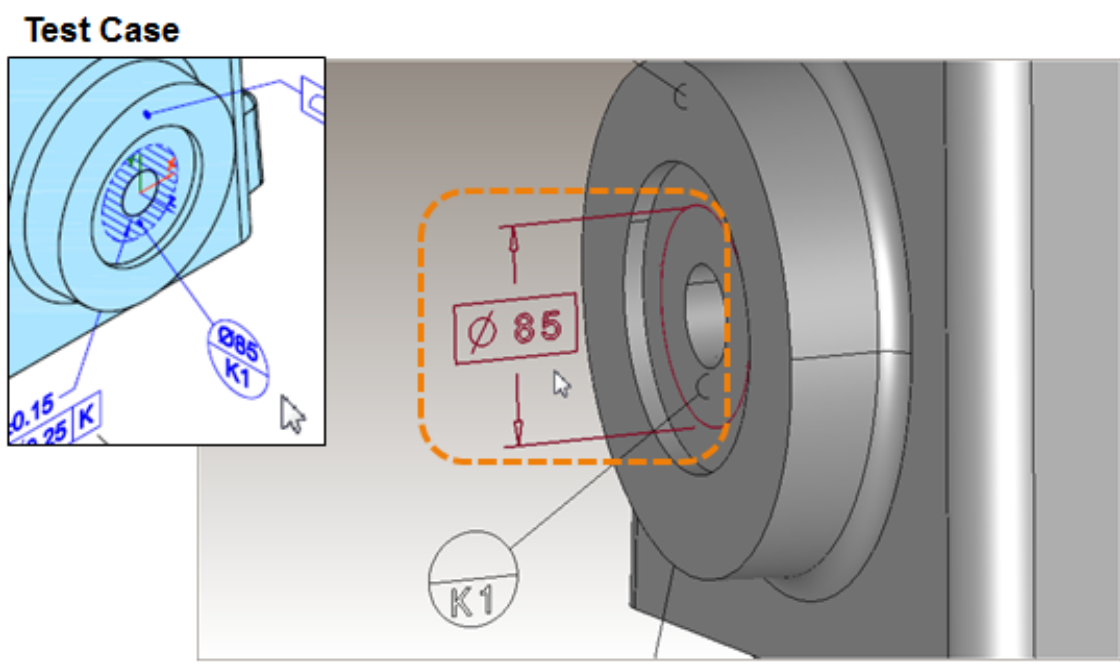

The diameter of this datum target area is defined as a separate dimension and not shown in the upper half of the datum target symbol as specified. 


\section{Annotation Layout: \\ Presentation Limitation \\ FCF text displayed above rather than below}

Test Case
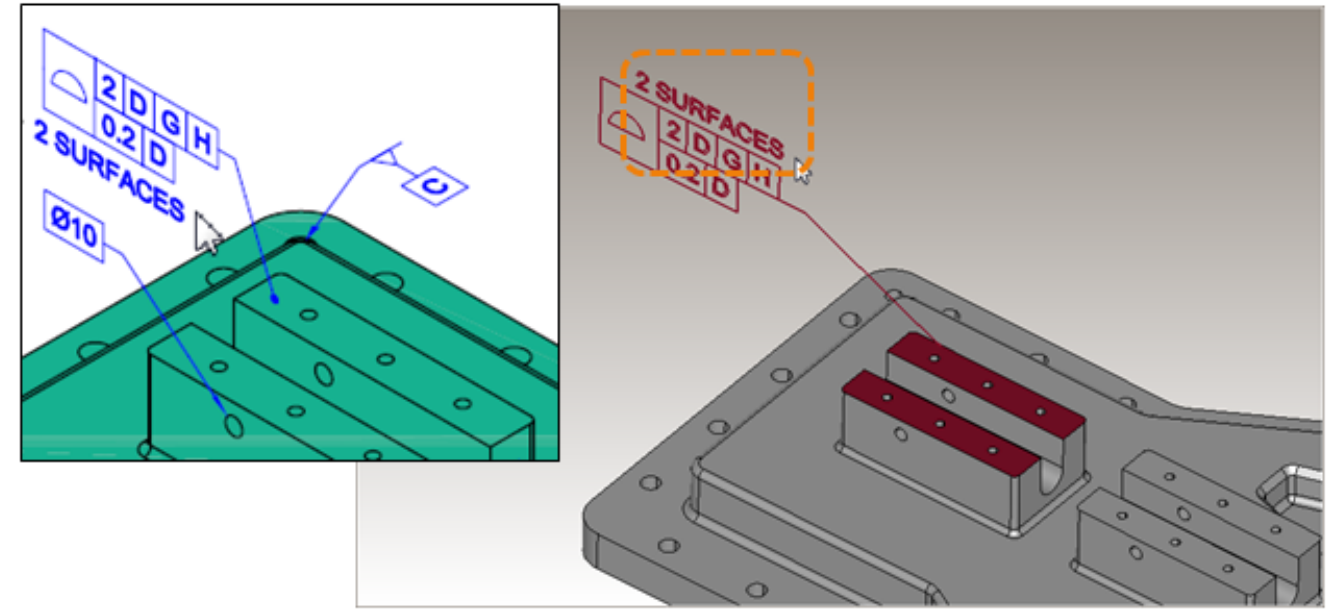

The "2 SURFACES" text is displayed above this feature control frame and not below as specified.

Annotation Layout:

Presentation Limitation FCF text displayed on right rather than below

Test Case

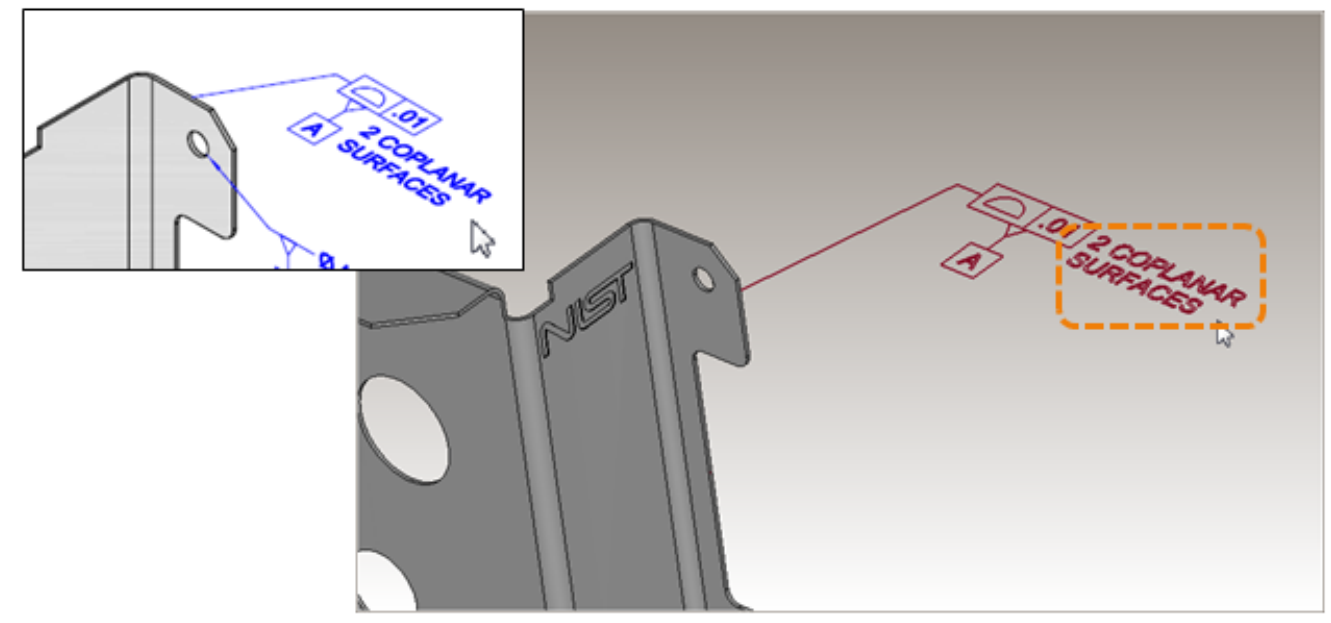

The "2 COPLANAR SURFACES" text is displayed on the right of this feature control frame and not below as specified. 


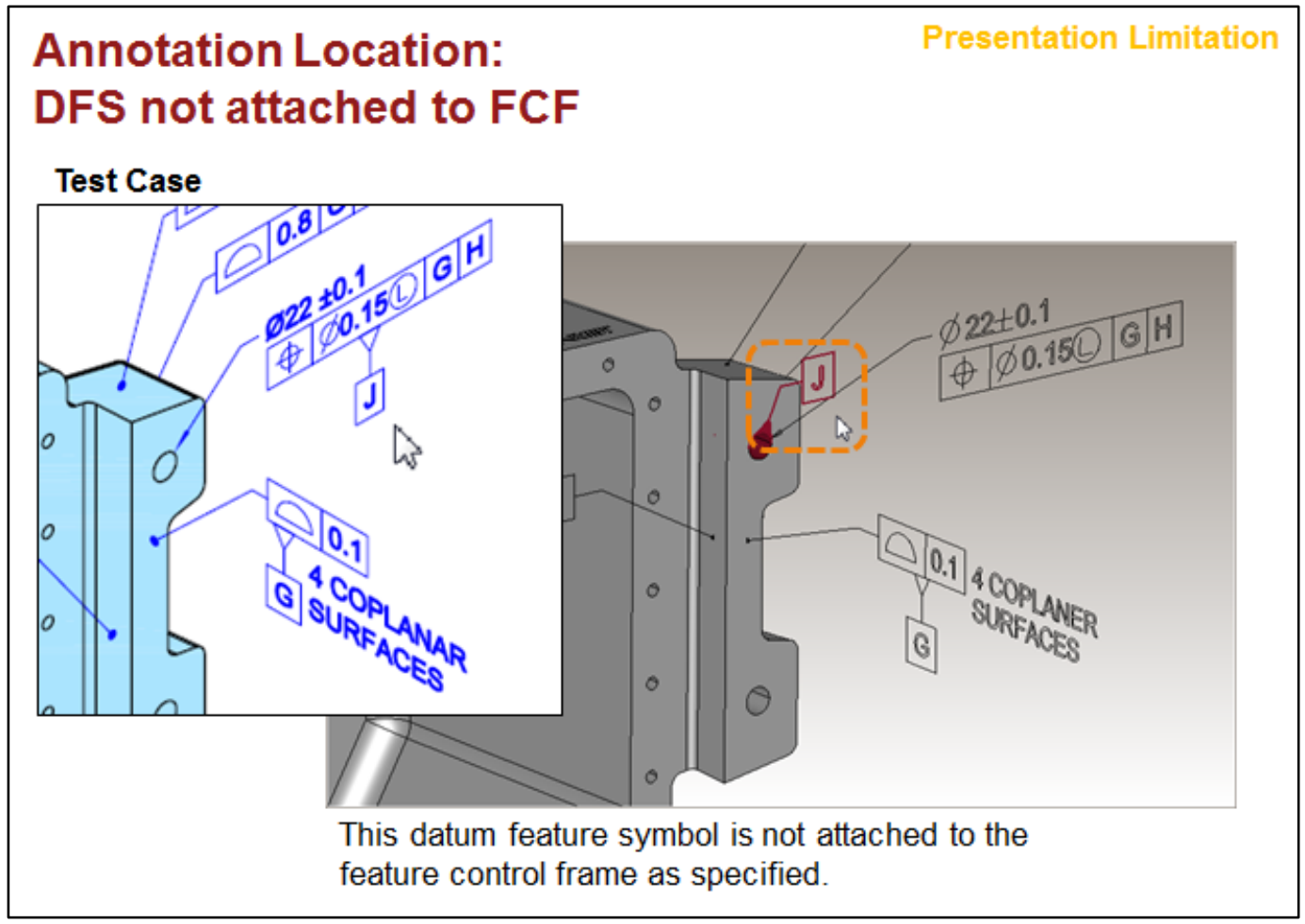

\section{Annotation Location: DFS overlaps DIM graphics}

Test Case

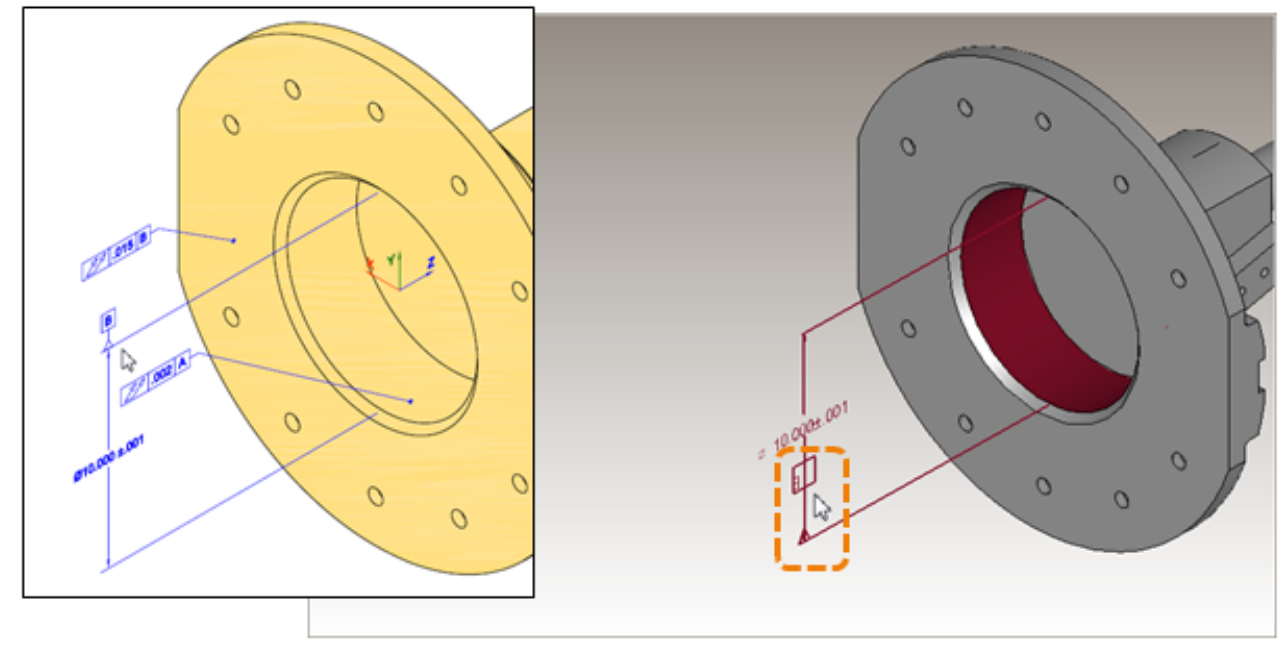

The display of this datum feature symbol overlaps the dimension to which it is attached. 
Annotation Location:

DFS partially buried in solid

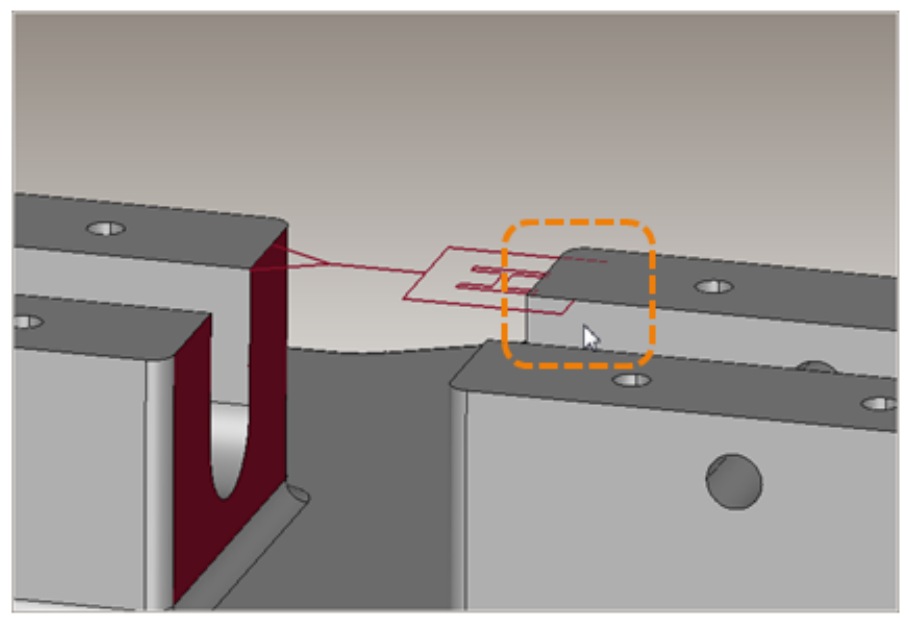

A portion of this datum feature symbol extends into the solid model, obscuring its display.

\section{Annotation Location:}

Presentation Limitation

\section{FCF partially buried in solid}

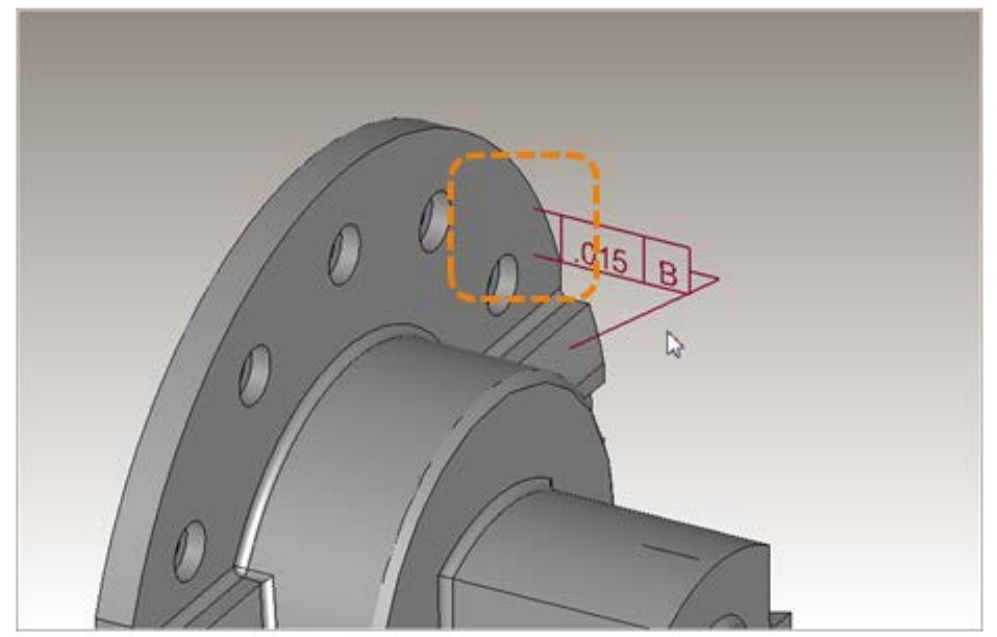

A portion of this feature control frame extends into the solid model, obscuring its display. 


\section{Annotation Orientation: \\ DIM text orientation is wrong}

Test Case
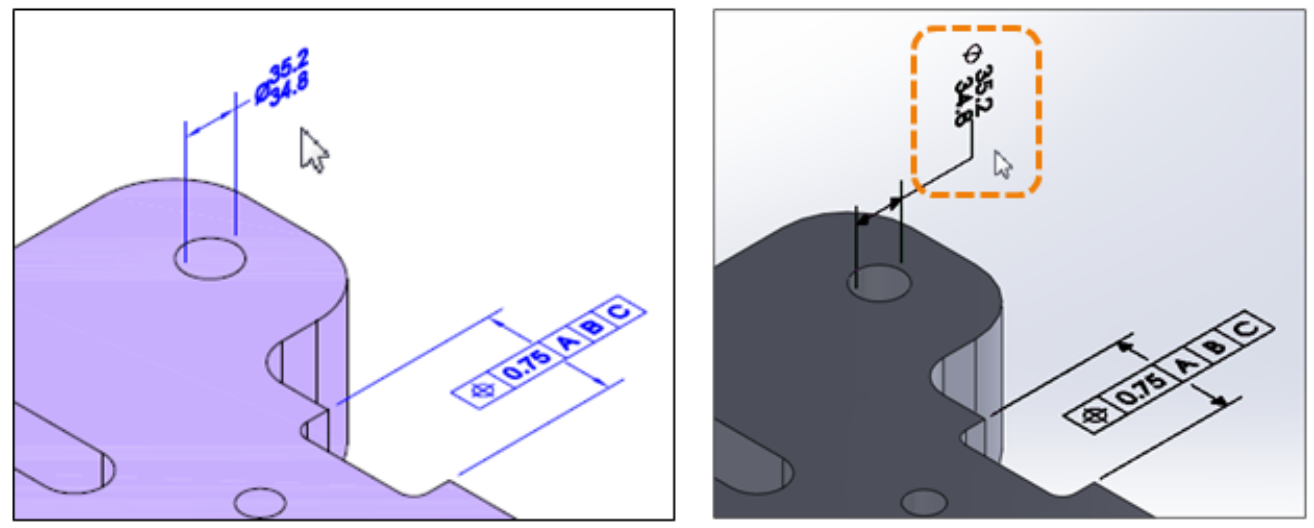

This dimension is not oriented horizontally as specified.

\section{Annotation Orientation:}

Presentation Limitation

\section{DTS text is backwards in this view}

\section{Test Case}
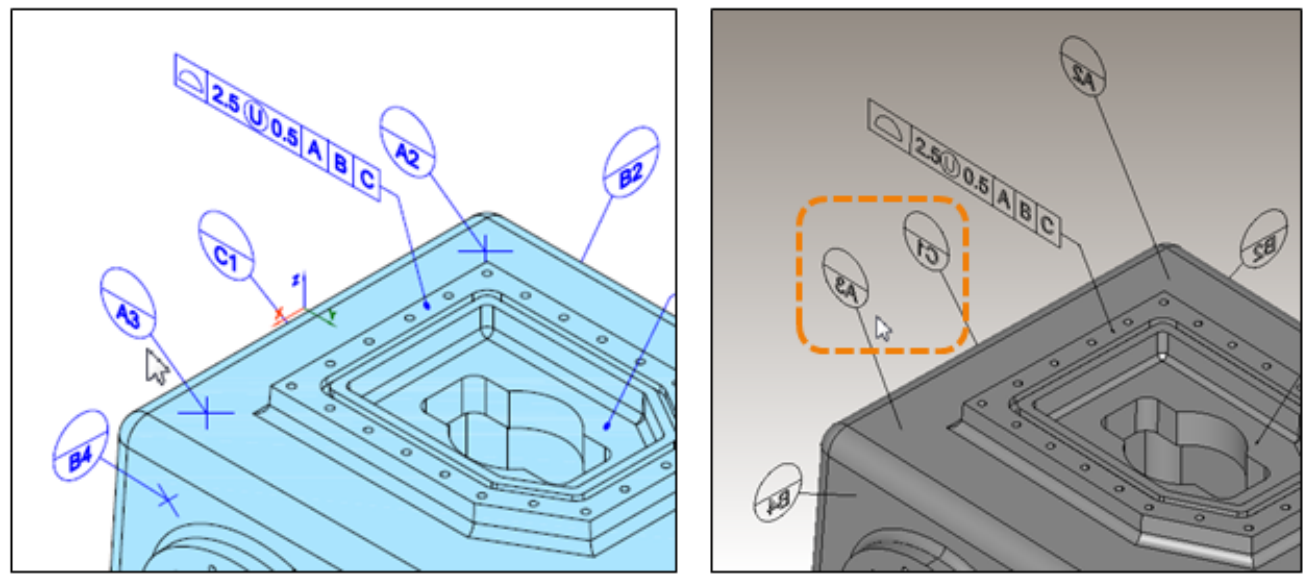

The read direction for the datum target symbols' text is backwards in this view from what is specified. 
Annotation Lines:

Presentation Limitation

\section{DFS has no extension line}

\section{Test Case}

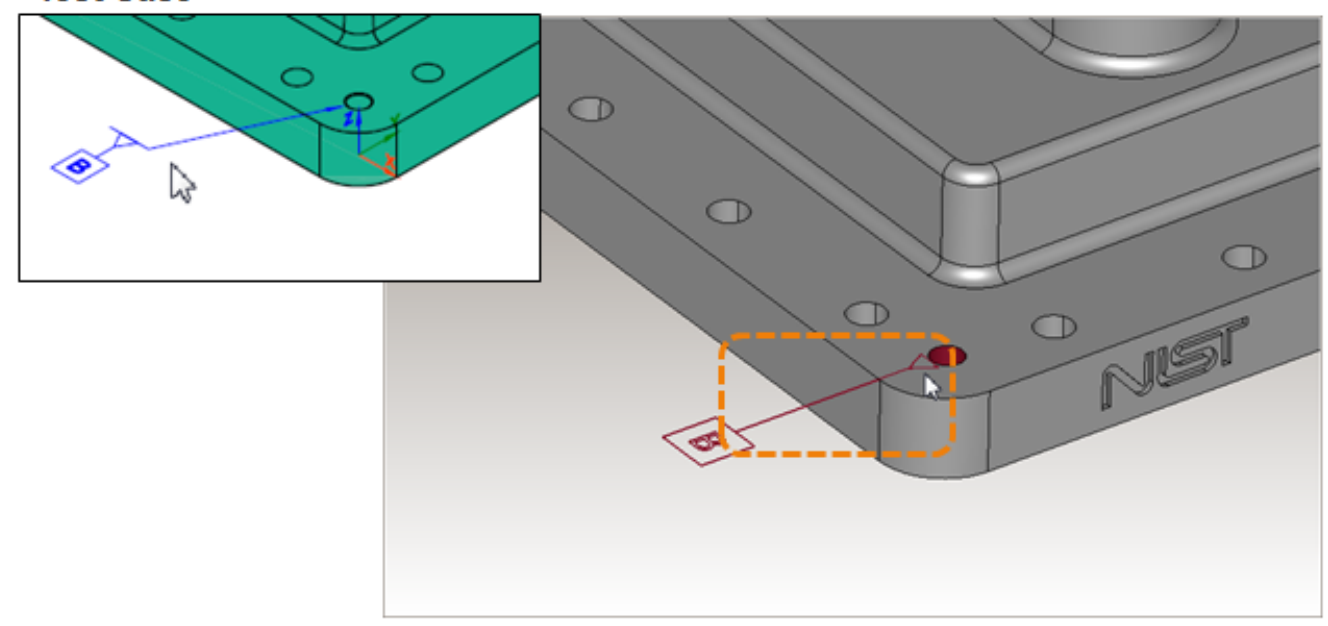

This datum feature symbol does not have the specified extension line.

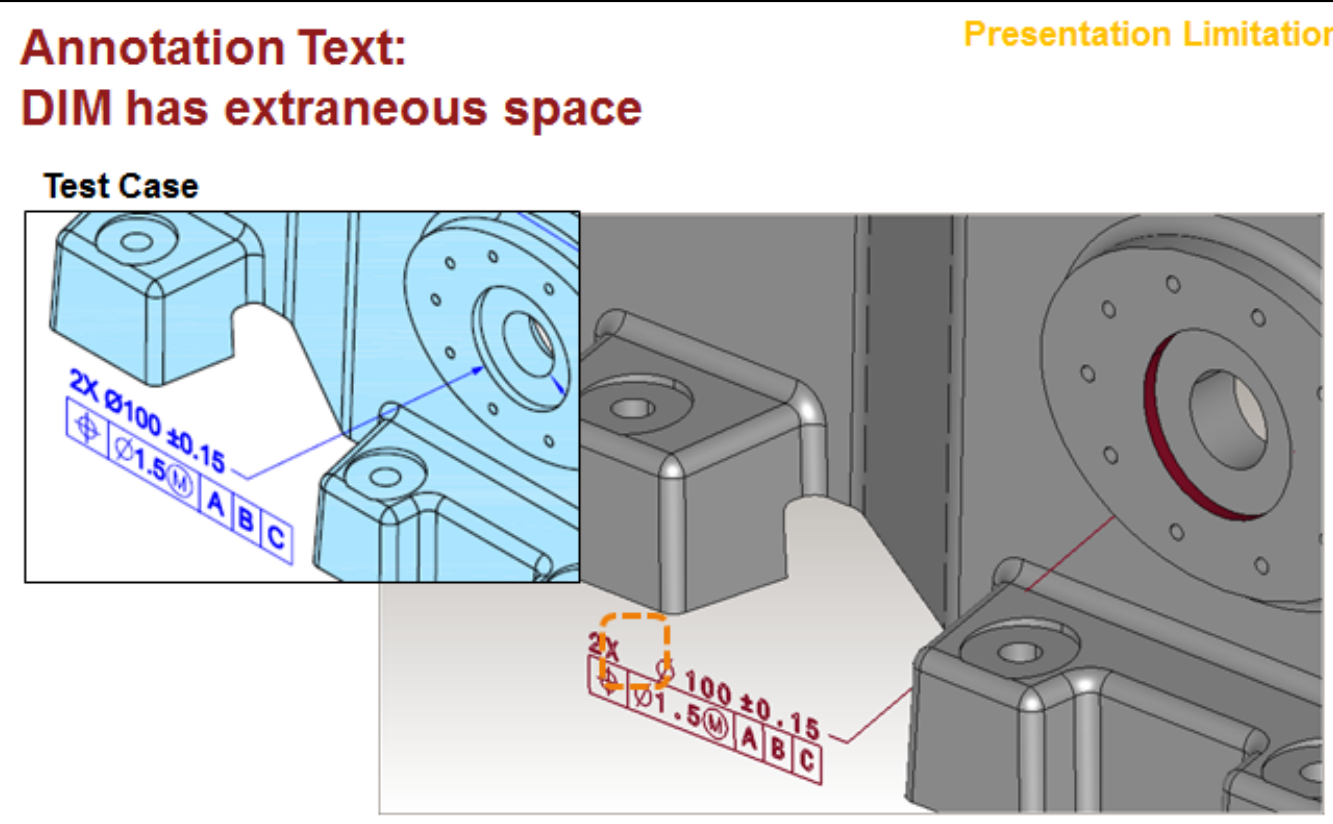

This dimension has an extra space after the pattern text $\left({ }^{u} 2 X^{n}\right)$ which is not specified. 


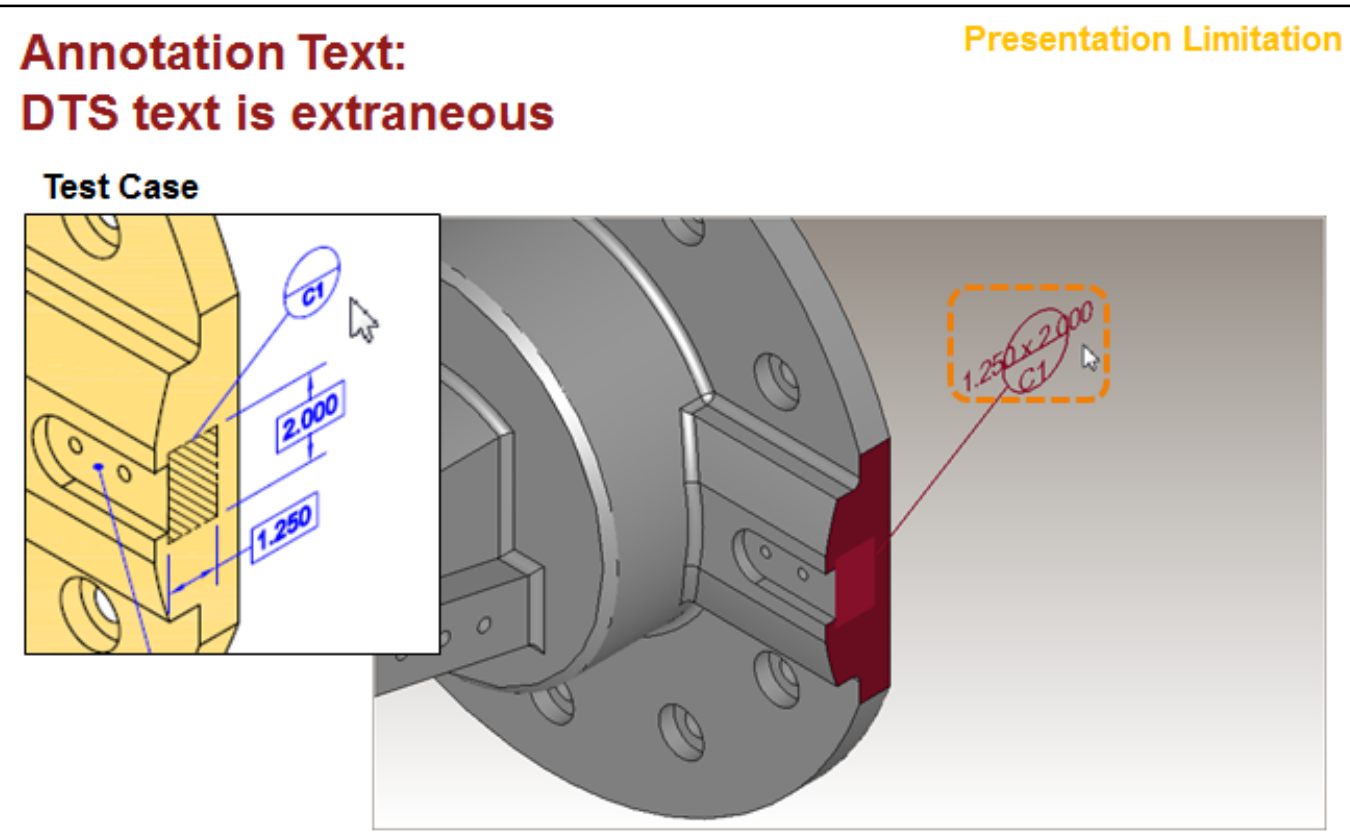

The target area dimensions shown in the upper half of this datum target symbol are not specified.

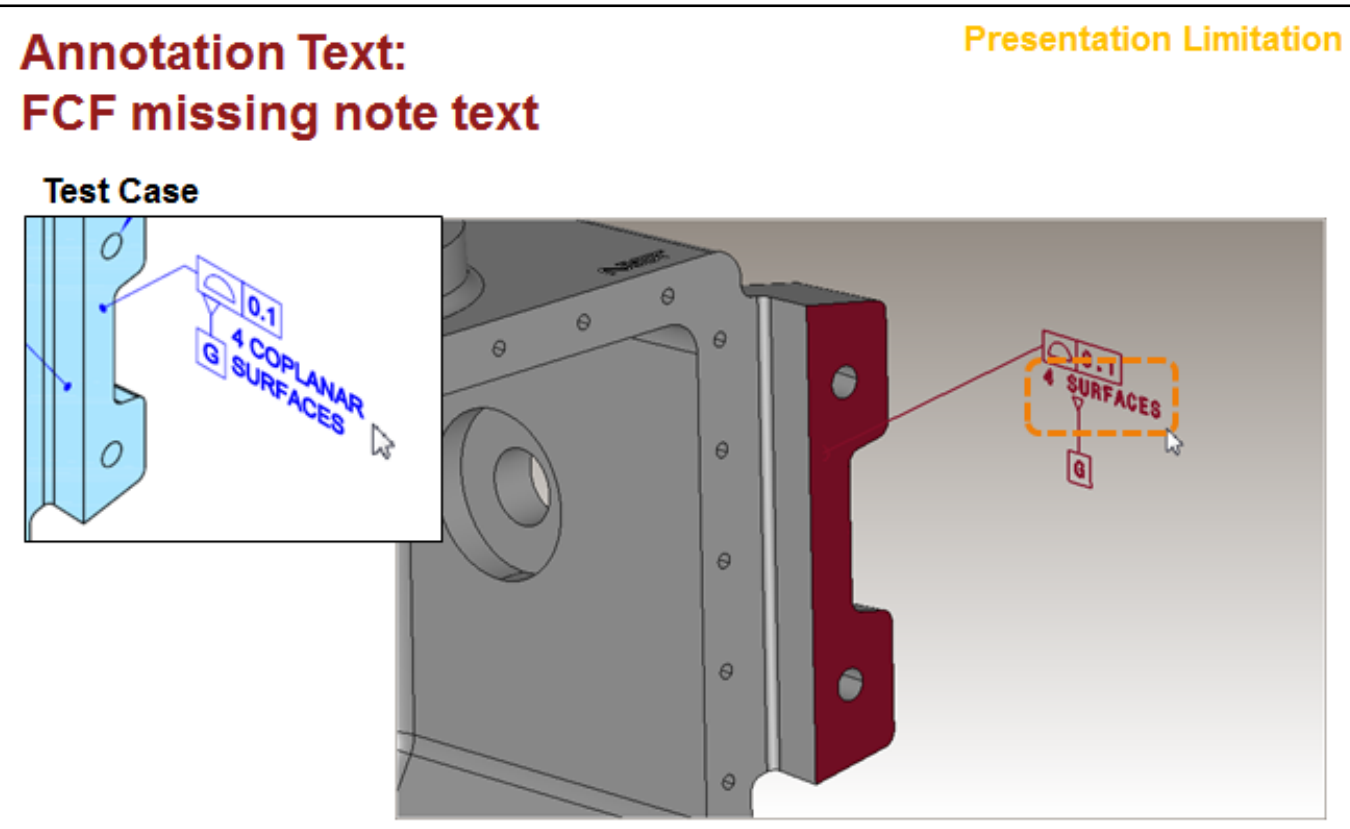

The specified "COPLANAR" text is missing for this feature control frame. 


\section{Annotation Text: \\ FCF missing projected tolerance zone length}

Test Case

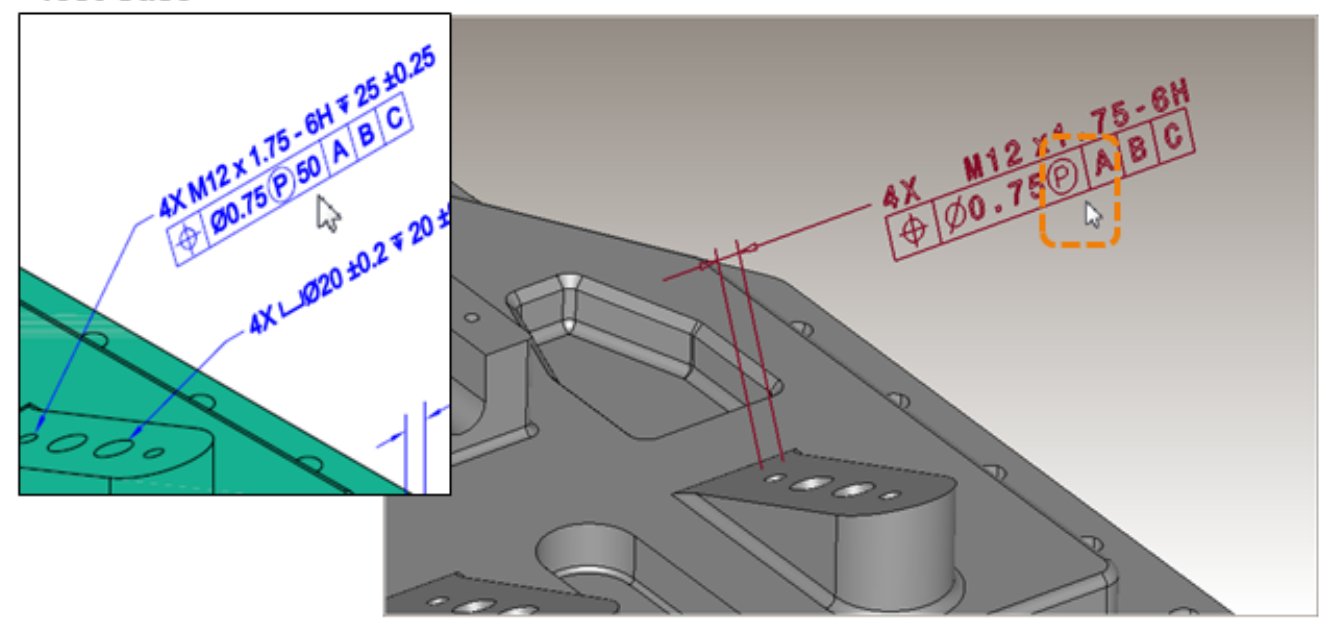

The length of the projected tolerance zone for this feature control frame is not display as specified.

\section{Annotation Text: \\ Presentation Limitation FCF text is extraneous}

Test Case

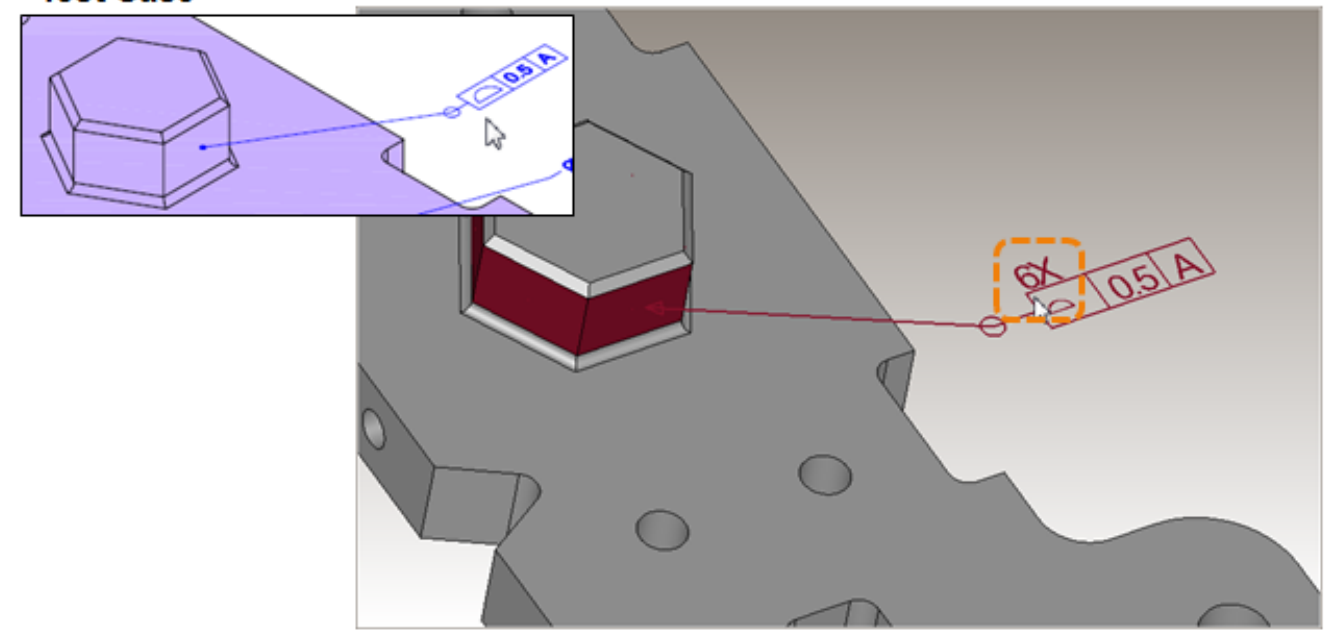

The " $6 \mathrm{X}$ " text above this feature control frame is not specified in the test case and is extraneous with the all-around symbol. 


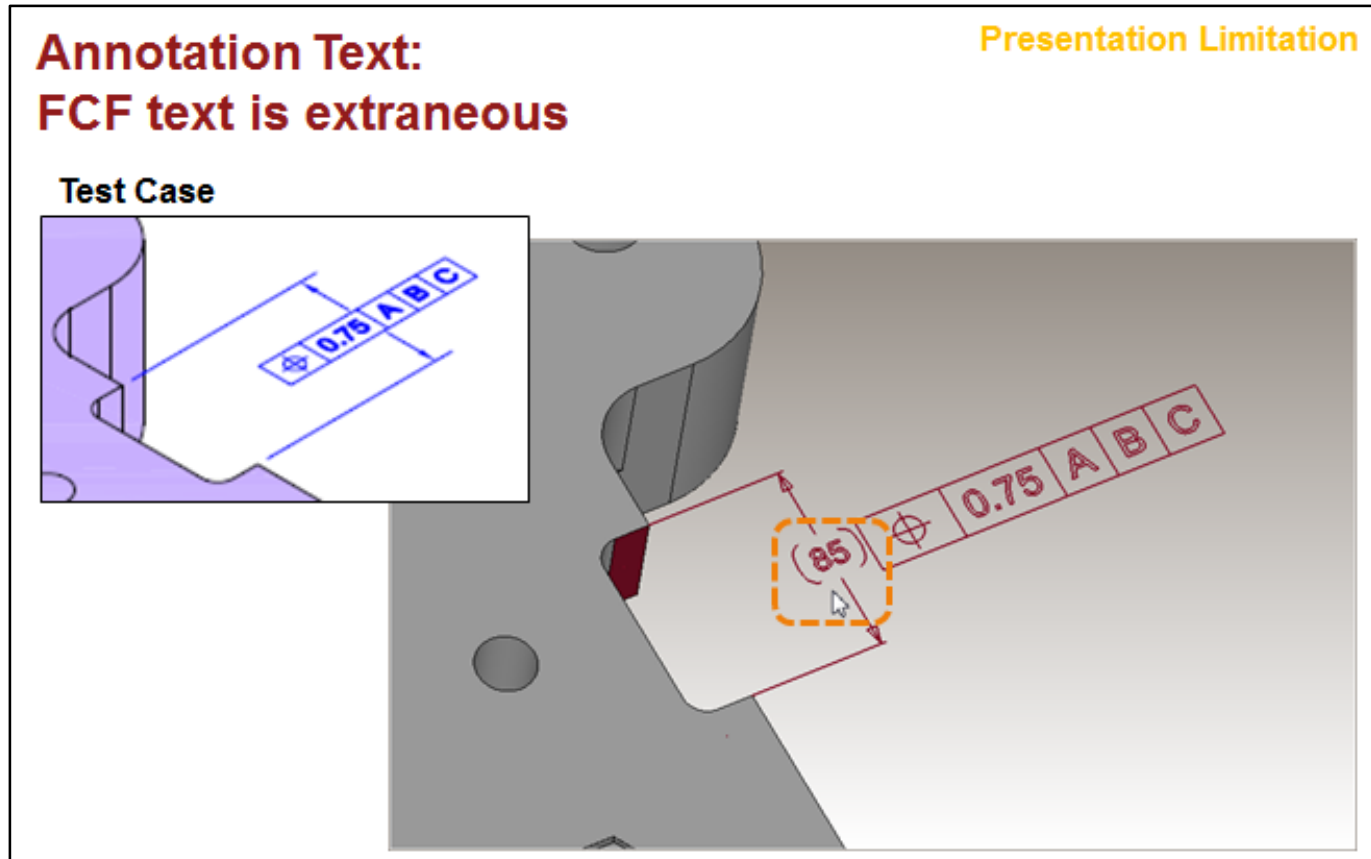

The " $(85)^{n}$ text on the left of this feature control frame is not specified in the test case.

\section{Coordinate System Visibility:} CS visible in wrong view

\section{Test Case}

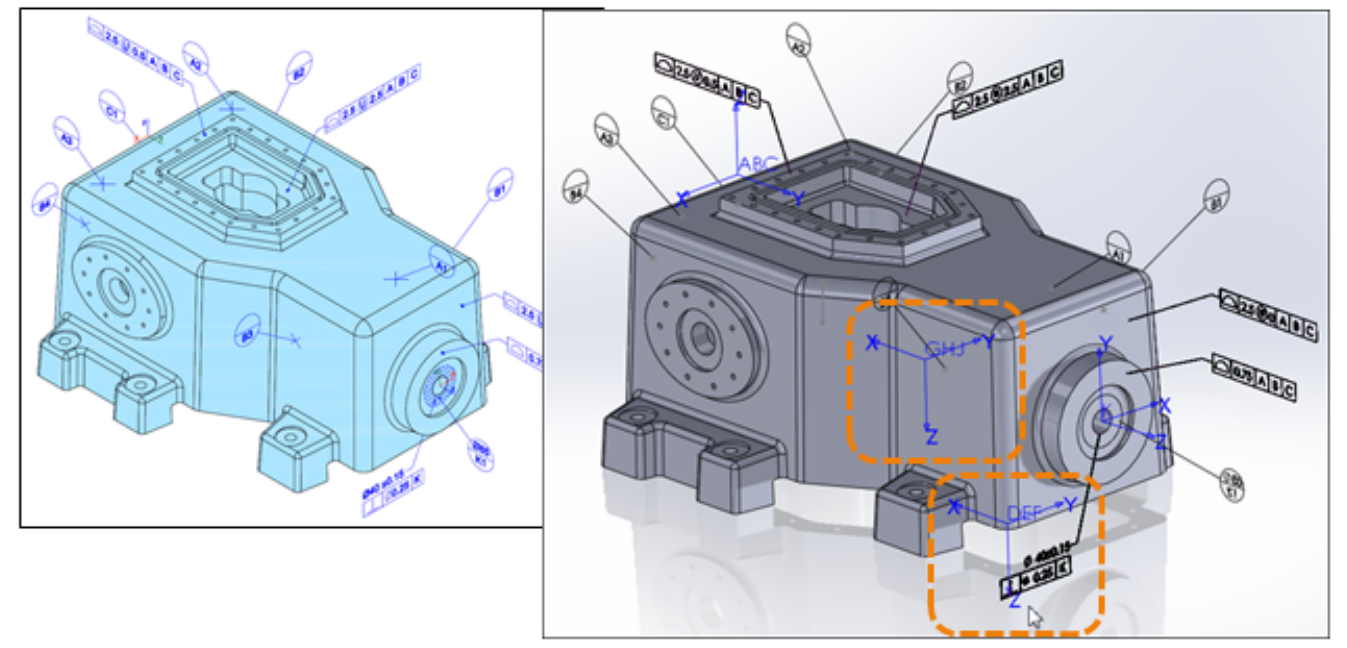

The "DEF" and "GHJ" coordinate systems are visible in a saved view in which they are not referenced. 
Coordinate System Name:

\section{CS name not same as DRF}
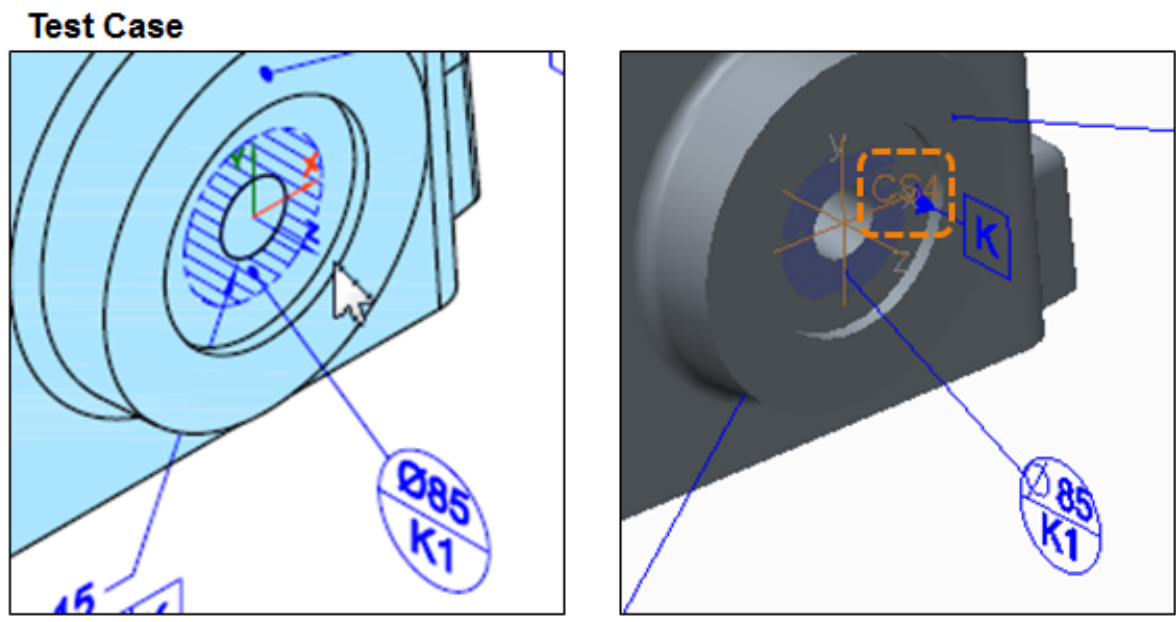

Because none of the systems enable an explicit link between annotations and coordinate systems, the name of each coordinate system should match its datum reference frame, thus providing an implicit (visual) link.

This coordinate system cannot be named with a single letter $\left({ }^{\prime} \mathrm{K}\right.$ ").

Coordinate System Text:

Presentation Limitation

\section{CS name displayed with extra large text}

\section{Test Case}
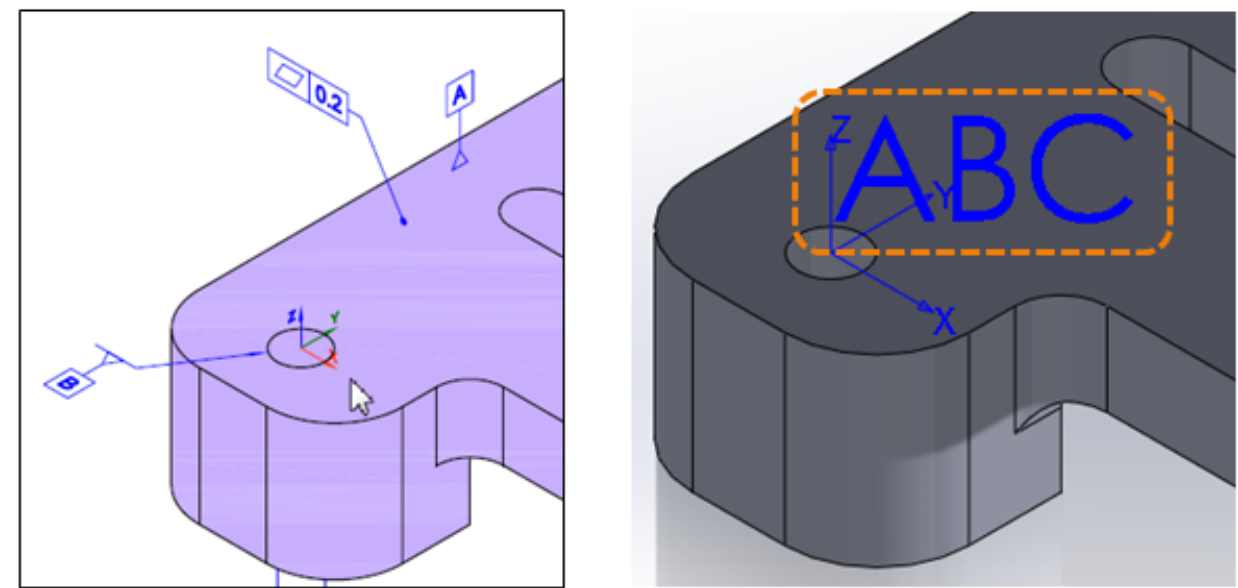

The display name for this coordinate system is extremely large. 


\section{Supplemental Geometry Visibility: Presentation Limitation SG curve visible in wrong view}

Test Case
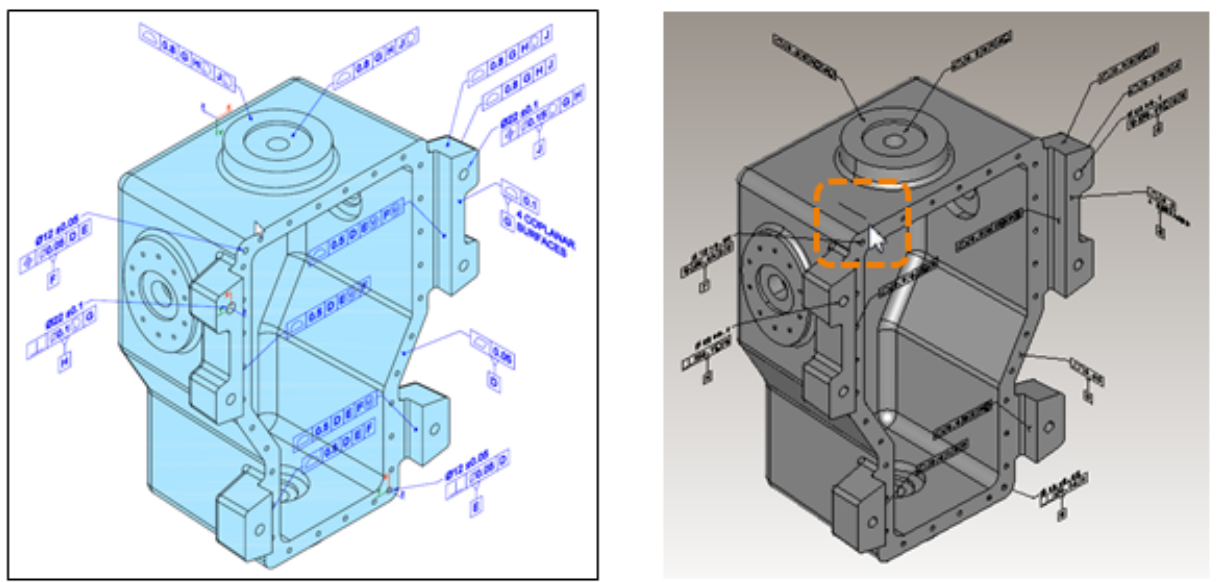

Because the profile of a line feature control frame in this model is not specified as visible in this saved view, then its associated supplemental geometry curve should not be visible.

\section{Supplemental Geometry Visibility: SG point visible in wrong view \\ Presentation Limitation}

Test Case

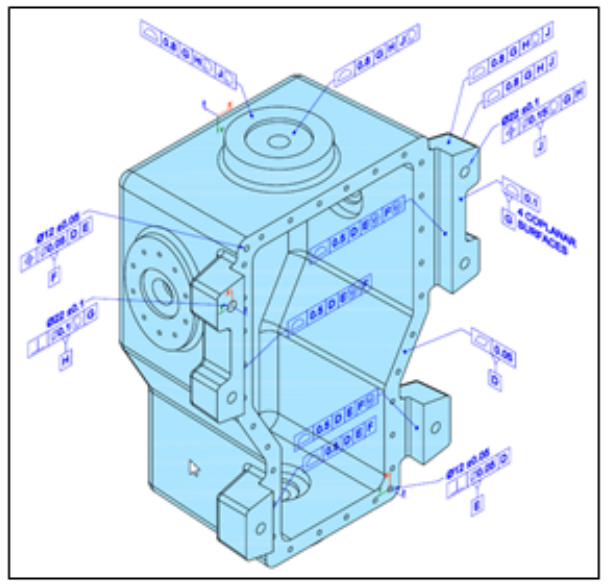

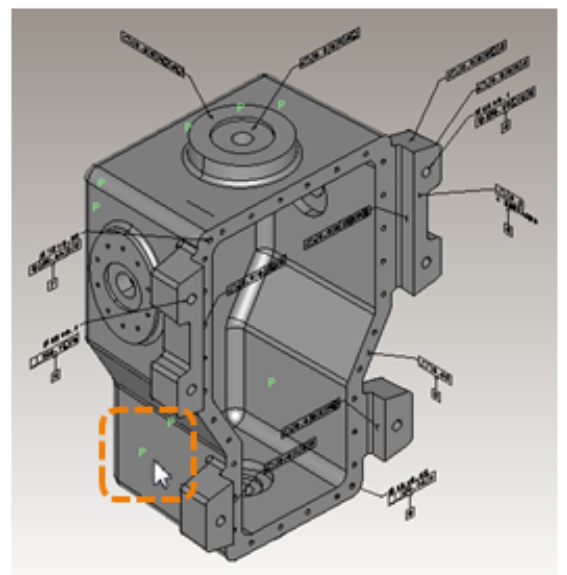

Because the datum targets for this model are not specified as visible in this saved view, then their associated supplemental geometry points should not be visible. 
Saved View Structure:

Presentation Limitation

View cannot contain annotations on different planes

Test Case
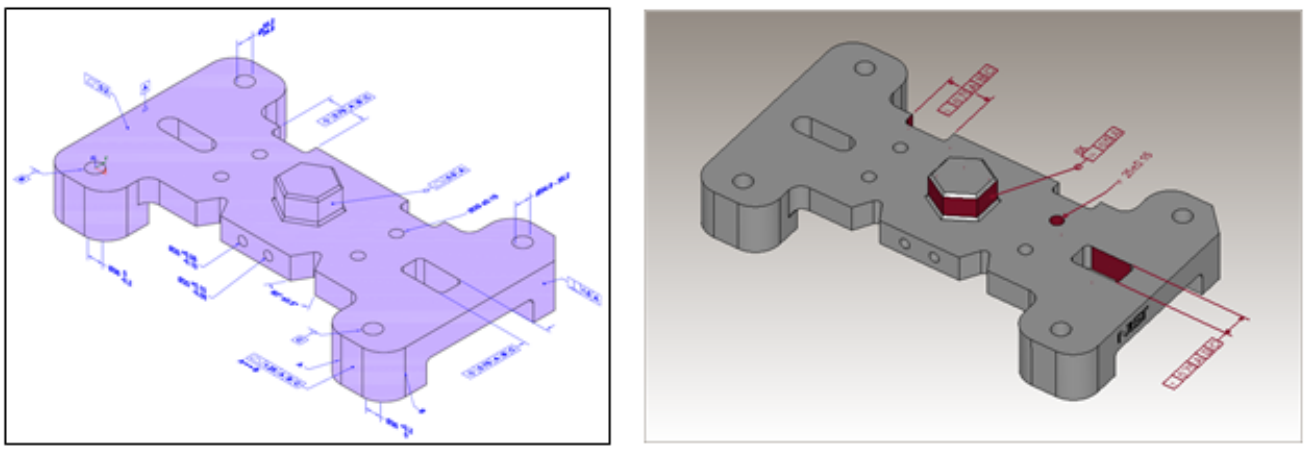

The PMI views in this system are limited to annotations with the same view and reading directions. The specified saved view has annotations with multiple view and reading directions.

Saved View Frustum:

Presentation Limitation View camera position not defined

Test Case

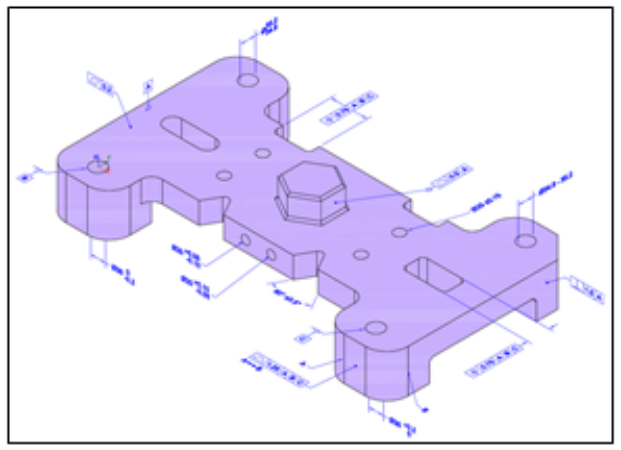

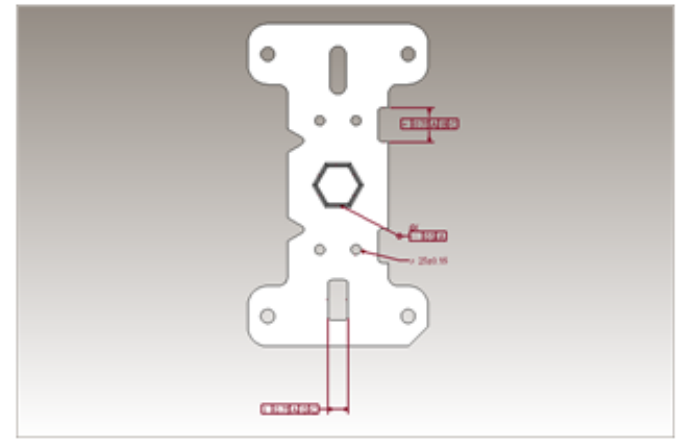

Each saved view in the test case has a specified camera position (view direction and zoom level). This system is unable to store a camera position in its PMI view definition. 
Appendix F: Style Difference Examples

Annotation Structure:

Style Difference DTS requires DFS to be defined

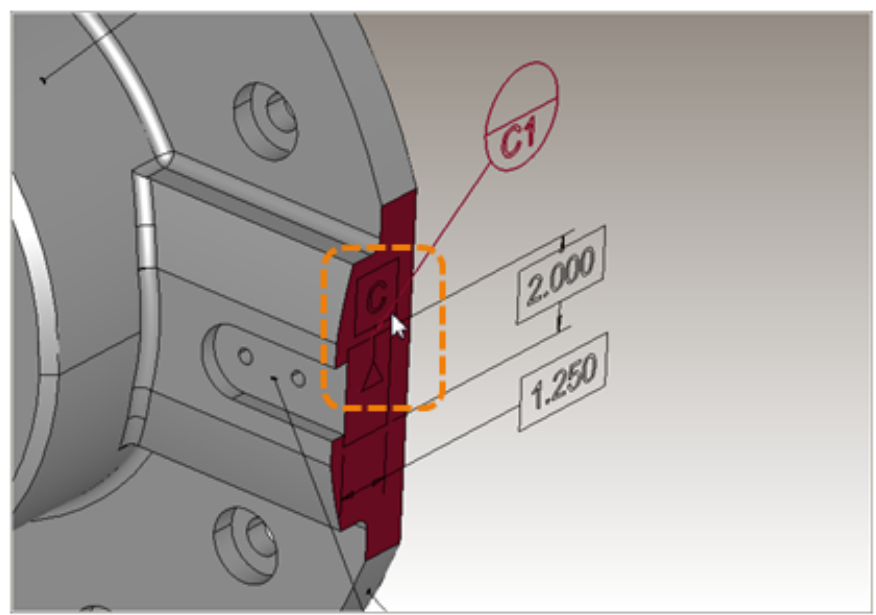

The system requires a datum feature symbol to be defined whenever a datum target symbol is defined.

\section{Annotation Structure:}

\section{FCF requires DFS to be defined}

\section{Test Case}

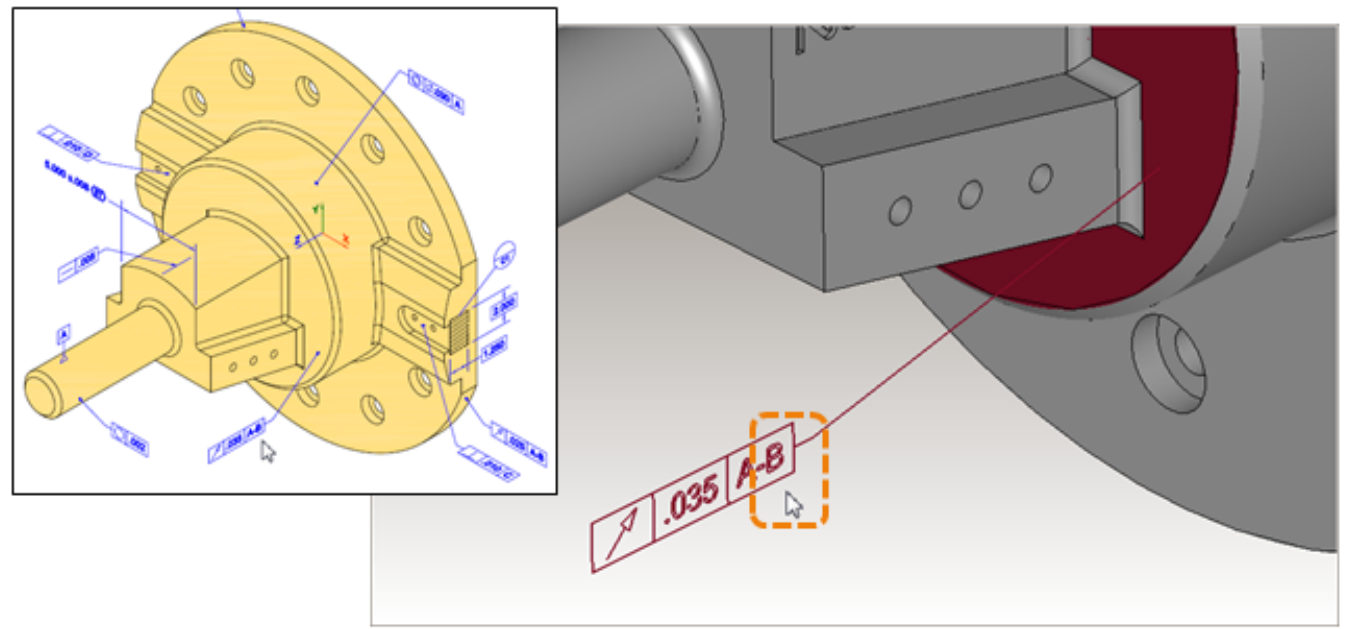

Because this feature control frame references datum " $\mathrm{B}$ ", its datum feature symbol must be defined in this saved view, although it can be hidden (not visible). 
Annotation Geometry:

\section{DFS edge association is extraneous}

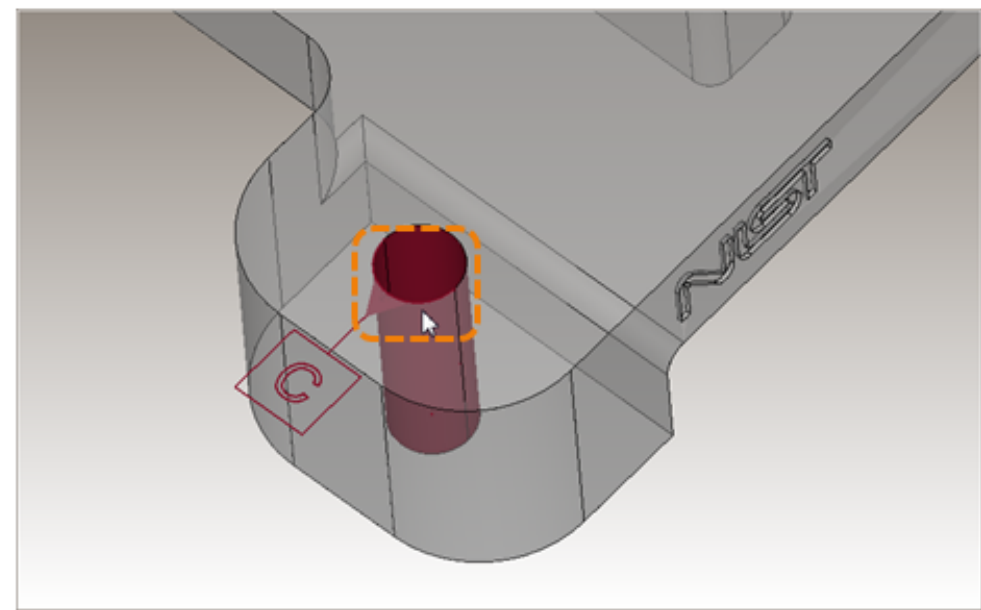

The association of this datum feature symbol with the edge of the hole is used to indicate graphical placement. It is not specified in the test case.

Annotation Geometry:

Style Difference

DIM edge association is extraneous

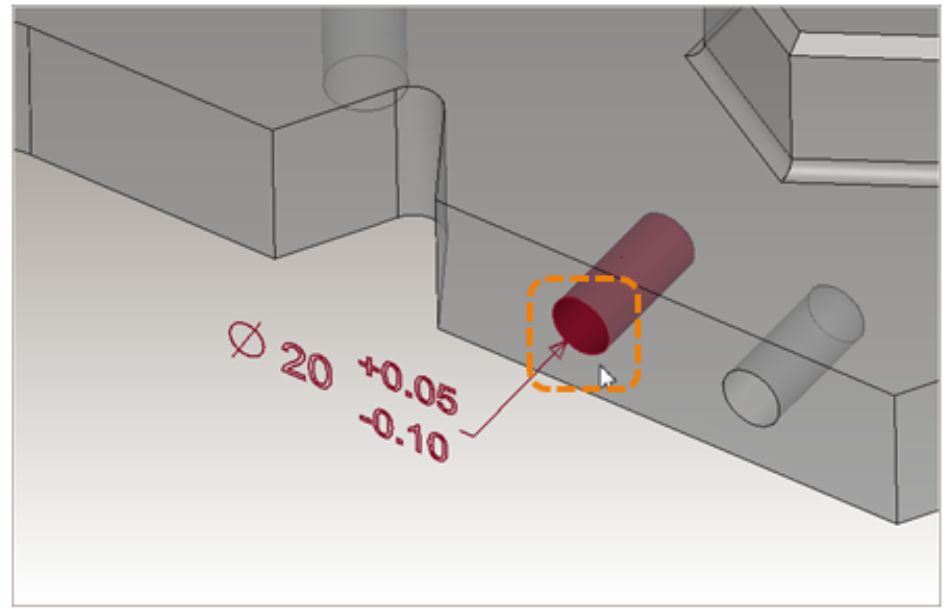

The association of this dimension with the edge of the hole is used to indicate graphical placement.

It is not specified in the test case. 
Supplemental Geometry Structure:

Style Difference

DTS target area is non-solid surface on solid face

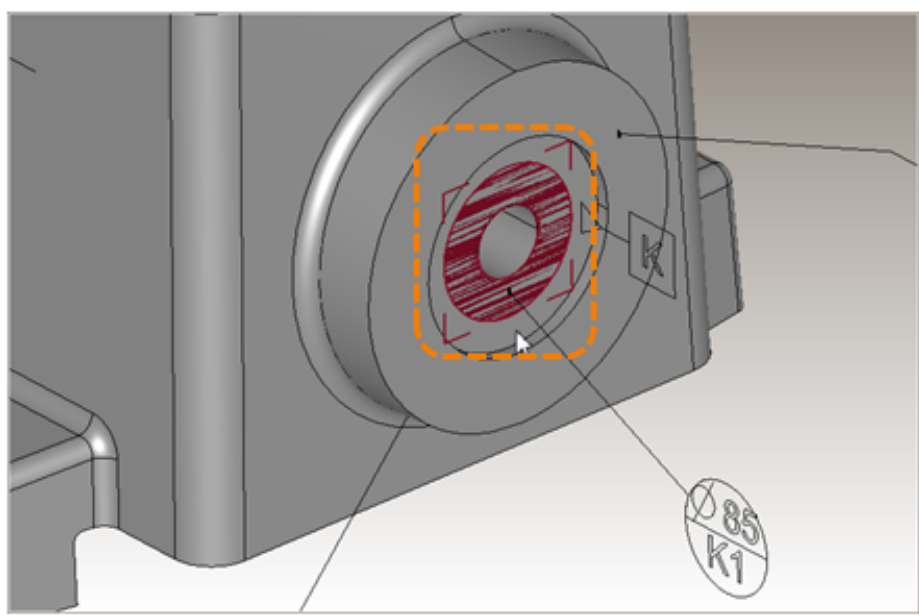

The target area for this datum target is defined as a non-solid surface placed on the solid face.

Supplemental Geometry Structure:

Style Difference DTS target area is subdivided solid face

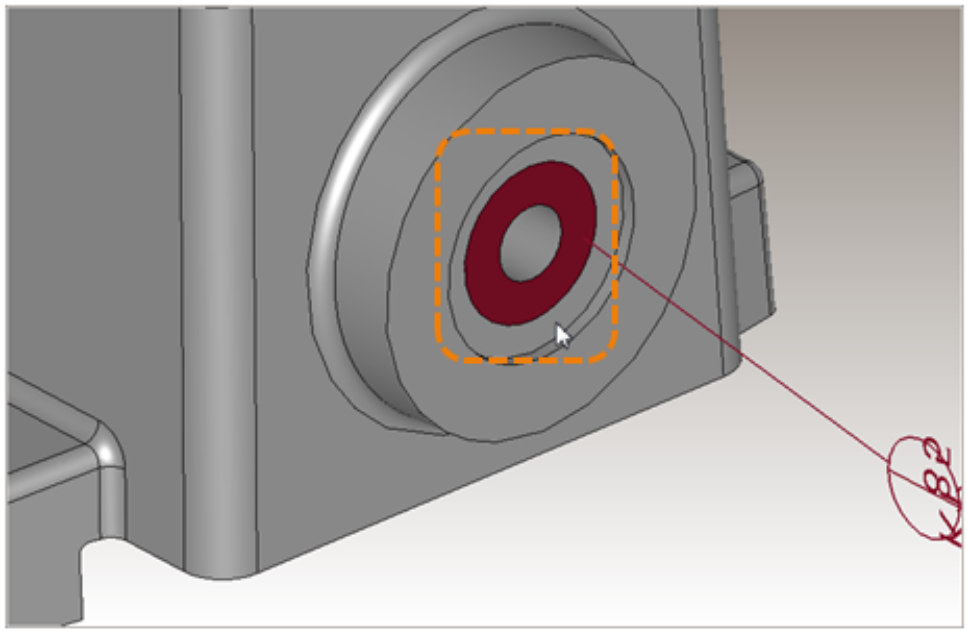

The target area for this datum target is defined as a solid face that has been separated from the adjacent faces in this solid. 
Supplemental Geometry Structure:

Style Difference

DTS target area is wireframe region on solid face

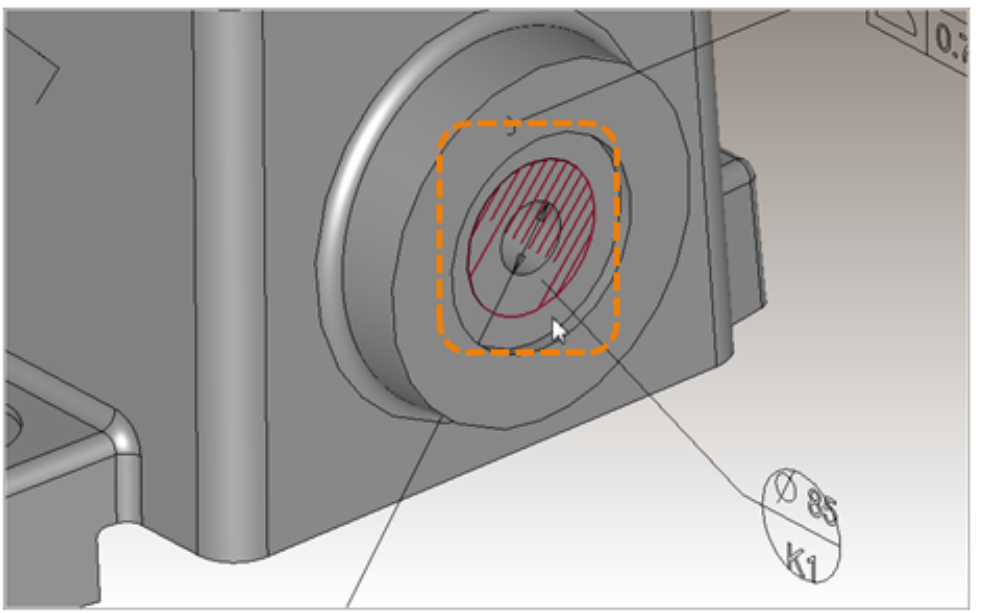

The target area for this datum target is defined as a wireframe region placed on the solid face.

Supplemental Geometry Structure:

Style Difference FCF limited area is non-solid surface on solid face

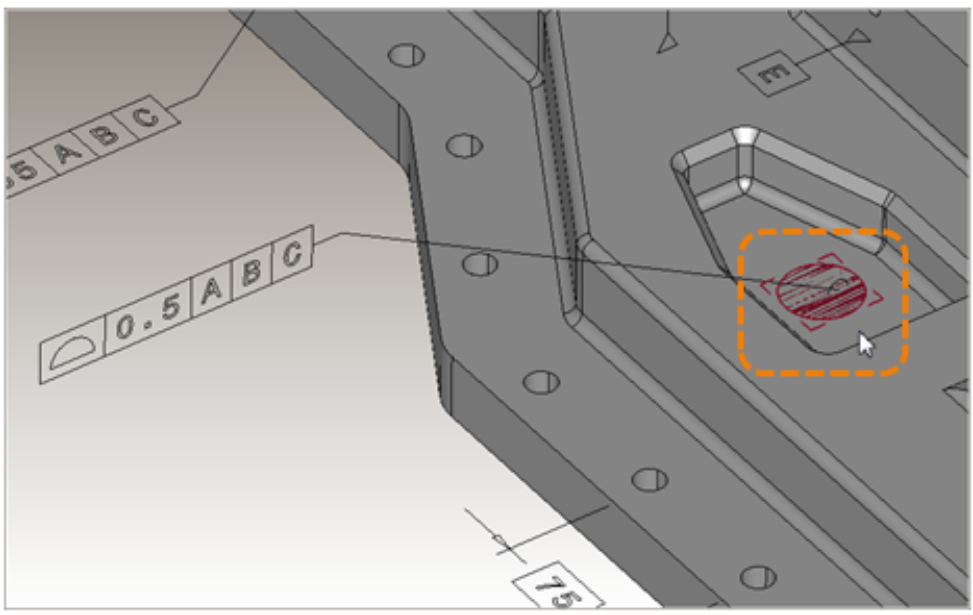

The limited area for this feature control frame is defined as a non-solid surface placed on the solid face. 
Supplemental Geometry Structure:

Style Difference FCF limited area is subdivided solid face

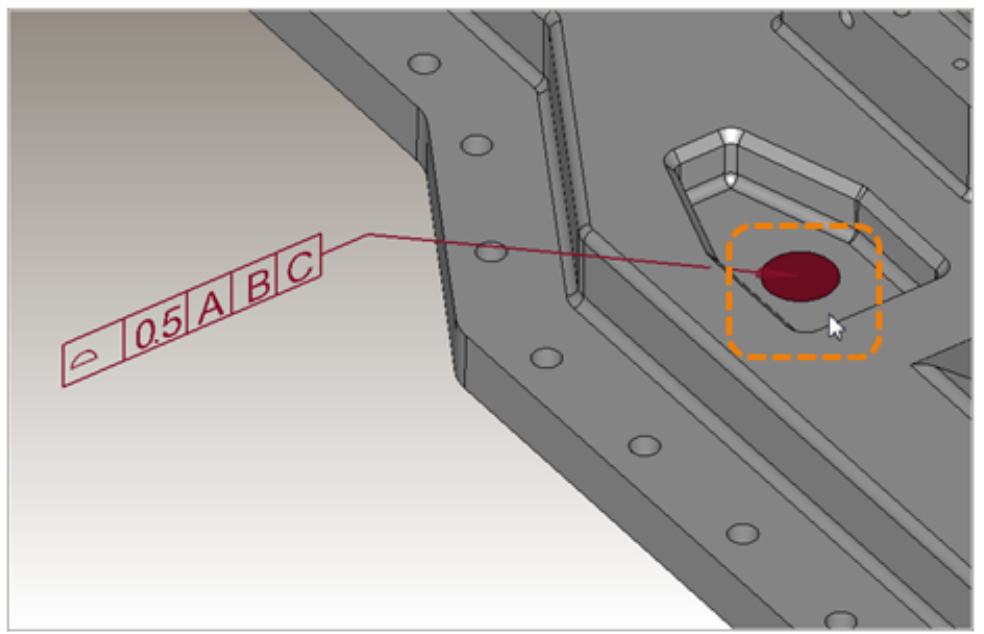

The limited area for this feature control frame is defined as a solid face that has been separated from the adjacent faces in this solid.

Supplemental Geometry Structure:

Style Difference

FCF limited area definition inconsistent with target area

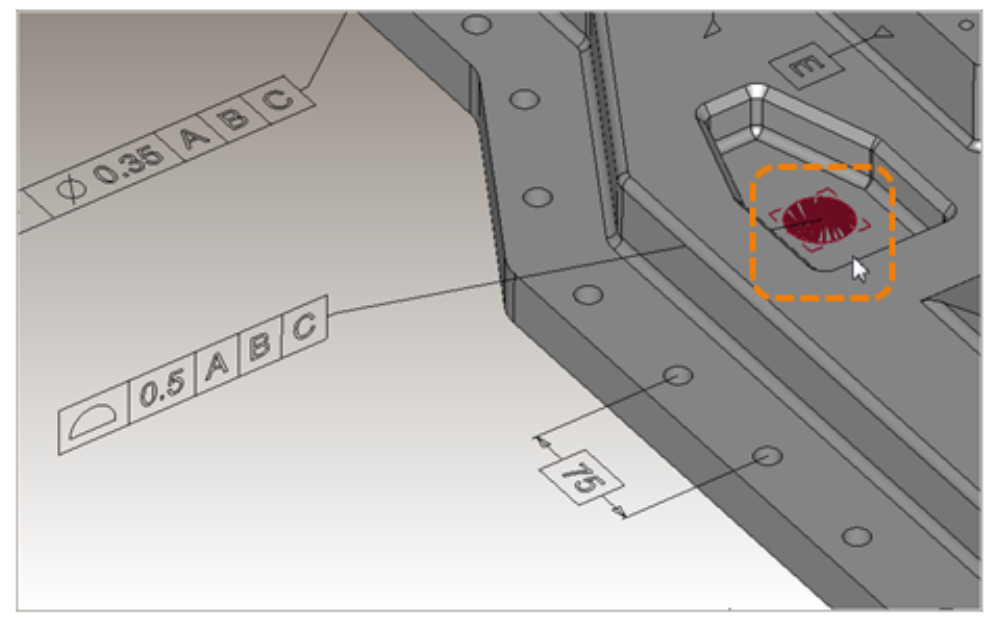

The limited area for this feature control frame is defined as a nonsolid surface placed on the solid face. This is inconsistent with the wireframe region used to define datum target areas in this system. 
Product Geometry Parameters:

Style Difference

Threaded hole diameter different than other systems

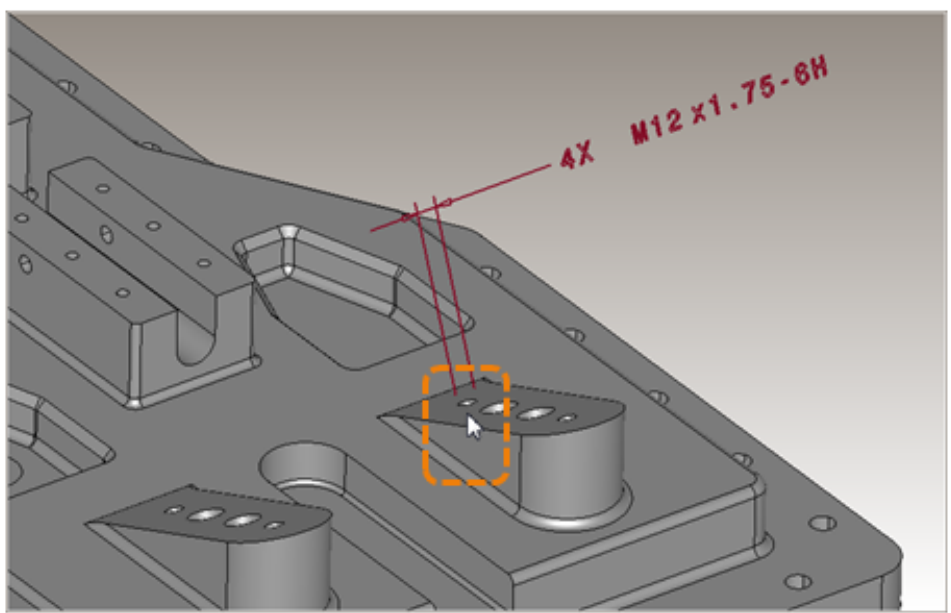

The nominal diameter of the geometric hole associated with this threaded hole diameter dimension is slightly different (by default) than in other systems. 